\title{
Overgroups of elementary block-diagonal subgroups in the classical symplectic group over an arbitrary commutative ring
}

\author{
Alexander Shchegolev \\ Saint Petersburg State University \\ iRyoka@gmail.com
}

\begin{abstract}
In this paper we prove a sandwich classification theorem for subgroups of the classical symplectic group over an arbitrary commutative ring $R$ that contain the elementary block-diagonal (or subsystem) subgroup $\operatorname{Ep}(\nu, R)$ corresponding to a unitary equivalence realation $\nu$ such that all self-conjugate equivalence classes of $\nu$ are of size at least 4 and all not-self-conjugate classes of $\nu$ are of size at least 5 . Namely, given a subgroup $H$ of $\operatorname{Sp}(2 n, R)$ such that $\operatorname{Ep}(\nu, R) \leq H$ we show that there exists a unique exact major form net of ideals $(\sigma, \Gamma)$ over $R$ such that $\operatorname{Ep}(\sigma, \Gamma) \leq H \leq \mathrm{N}_{\mathrm{Sp}(2 n, R)}(\operatorname{Sp}(\sigma, \Gamma))$. Further, we describe the normalizer $\mathrm{N}_{\mathrm{Sp}(2 n, R)}(\mathrm{Sp}(\sigma, \Gamma))$ in terms of congruences.
\end{abstract}

\section{Introduction}

This paper describes the overgroups $H$ of the elementary symplectic block-diagonal subgroup $\operatorname{Ep}(\nu, R)$ of type $\nu$ of the classical symplectic group $\operatorname{Sp}(2 n, R)$ over a commutative ring $R$, under the assumption that the minimal size of a self-conjugate block of $\operatorname{Ep}(\nu, R)$ is at least 4 and the minimal size of a non-self-conjugate block of $\operatorname{Ep}(\nu, R)$ is at least 5 . The main result is the following sandwich classification theorem: let $\nu, R, H, \operatorname{Ep}(\nu, R)$ and $\operatorname{Sp}(2 n, R)$ be as above. Then there exists a unique major exact form net of ideals $(\sigma, \Gamma)$ over the ground ring such that $H$ fits into the sandwich

$$
\operatorname{Ep}(\sigma, \Gamma) \leq H \leq \mathrm{N}_{\mathrm{Sp}(2 n, R)}(\operatorname{Sp}(\sigma, \Gamma)),
$$

where $\mathrm{N}_{\mathrm{Sp}(2 n, R)}(\operatorname{Sp}(\sigma, \Gamma))$ denotes the normalizer in $\operatorname{Sp}(2 n, R)$ of the form net subgroup $\operatorname{Sp}(\sigma, \Gamma)$ of $\operatorname{Sp}(2 n, R)$ of level $(\sigma, \Gamma)$ and $\operatorname{Ep}(\sigma, \Gamma)$ the elementary form net subgroup of $\operatorname{Sp}(\sigma, \Gamma)$.

Research is supported by the Russian Science Foundation grant 17-11-01261. 
To put this result into context we provide a brief overview of related results describing the subgroup structure of linear groups over fields. In [Dyn57a, Dyn57b Dynkin determined the maximal closed connected subgroups of classical algebraic groups over $\mathbb{C}$. In particular, he showed that all reductive maximal closed connected subgroups are precisely the stabilizers of totally isotropic or non-degenerate subspaces. Similar results for classical groups over algebraically closed fields of positive characteristic were obtained by Gary Seitz [Sei87] and for exceptional groups by Donna Testerman [Tes88]. In the papers Asc84, Asc85, Asc86] Michael Aschbacher described the maximal subgroups of finite simple classical groups. The subgroup structure theorem of Aschbacher says that every maximal subgroup of a finite simple classical group belongs to either one of 8 explicitly defined classes $\mathcal{C}_{1}-\mathcal{C}_{8}$ of large subgroups or to the class $\mathcal{S}$ of almost simple groups in irreducible representations. An exposition of results regarding the members of Aschbacher classes in finite classical groups can be found in the book of Kleidman and Liebeck [KL90]. The Aschbacher classes which are relevant for us are the classes $\mathcal{C}_{1}$ and $\mathcal{C}_{2}$. The subgroups of class $\mathcal{C}_{1}$ are stabilizers of proper totally isotropic or nondegenerate submodules of the module on which the group is acting. The subgroups of class $\mathcal{C}_{2}$ are the stabilizers of direct decompositions of that module into the summands of a fixed dimension. Given a member $H$ of an Aschbacher class the book of Kleidman and Liebeck provides a recipe for constructing a maximal overgroup of $H$ that is in turn also a member of some not necessarily the same Aschbacher class. Unfortunately, a similar result for classical groups over arbitrary commutative rings (or unitary groups over form rings) has not yet been obtained. However, it is possible to describe the lattice structure of the set of all overgroups of a given member of an Aschbacher class or of an appropriate modified notion thereof in terms of the structure of the ground ring.

The literature contains several modifications of the notion of Aschbacher classes. The current paper focuses on a specific simultaneous modification of the Aschbacher classes $\mathcal{C}_{1}$ and $\mathcal{C}_{2}$ which we call block-diagonal subgroups. These subgroups are the stabilizers of certain direct decompositions of the quadratic module on which the unitary group is acting into totally isotropic or non-degenerate submodules. As our methods only employ the elementary matrices contained in these block-diagonal subgroups, we will describe the overgroups of elementary block-diagonal subgroups instead of full blockdiagonal subgroups. The problem of describing overgroups of elementary block-diagonal matrices was first considered for the case of the general linear group in papers [BVN70, BV82, Vav83, BV84, Vav87] of Z.I. Borewicz, N. A. Vavilov and W. Narkiewicz over commutative rings and rings satisfying a stable rank condition. These papers do not use localization. Over quasi-finite rings the problem is solved in BS01 using localization. For the case of other classical groups over a commutative ring with 2 invertible, this classification was generalized in chapter $\mathrm{V}$ of the habilitation of Nikolai Vavilov, although complete proofs were only published much later in [Vav04 for the split orthogonal case and [Vav08] for the symplectic case. Important auxiliary results can be also found in Vav93, Vav88 and in the references therein. Roughly the main results in the above references can be described as follows. Let $\mathrm{G}$ denote a Chevalley group of type $A_{l}, B_{l}, C_{l}$ or $D_{l}$ over a commutative ring $R$ or the general linear group $\mathrm{GL}(n, R)$ over a quasi-finite ring $R$. If $\mathrm{G} \neq \mathrm{G}\left(A_{l}, R\right)$ or $\mathrm{GL}(n, R)$, assume that $2 \in R^{*}$. Let $H$ be a subgroup of 
G containing an elementary block-diagonal subgroup of $G$ whose minimal block size is sufficiently large. Then there exists a unique net of ideals $\sigma$ such that $H$ fits into the sandwich

$$
\mathrm{E}(\sigma) \leq H \leq \mathrm{N}(\sigma)
$$

where $\mathrm{E}(\sigma)$ is the elementary subgroup associated with $\sigma$ and $\mathrm{N}(\sigma)$ is the normalizer in $G$ of the net subgroup $\mathrm{G}(\sigma)$. In [BV84] such a description was called standard. We shall refer to it as standard sandwich classification.

Unfortunately, due to known counterexamples the standard sandwich classification in Vav08] for the symplectic group $\operatorname{Sp}(2 n, R)$ when 2 is invertible in the ground ring $R$ does not generalize to the case of an arbitrary commutative ring. The obstacle is that the notion of a net of ideals is not fine enough when 2 is not invertible in the ring and has to be replaced by the notion of a form net of ideals. This is analogous to the situation encountered in the sandwich classification of subgroups of Bak unitary groups [Bak69, BV00], which are normalized by the elementary subgroup. Here the notion of ideal had to be refined by the notion of form ideal when 2 is not invertible in the ground ring. Significant work on developing the concept of a form net of ideals in the context of even unitary groups over fields and simple Artinian rings was done already by E. Dybkova Dyb98, Dyb99, Dyb06, Dyb07, Dyb09. Further review of known results on the problem of describing overgroups of subsystem subgroups in classical-like and some exceptional groups can be found in [VS13]. As mentioned there, no steps have yet been taken towards describing overgroups of subsystem subgroups in classical (other than $G L)$ groups over an arbitrary commutative ring or Bak unitary groups over form rings other than simple Artinian form rings.

This paper is the first one in the series devoted to the describing the overgroups of elementary block-diagonal subgroups of even unitary groups over quasi-finite rings which constitutes the $\mathrm{PhD}$ thesis [Shc15] of the author. The statement of the result for the even unitary case can also be found in [Shc17. Now we state the main result of this paper.

Let $R$ denote a commutative ring and $\operatorname{Sp}(2 n, R)$ the classical simplectic group with coefficients in $R$. Let $\operatorname{Ep}(2 n, R)$ denote the elementary subgroup of $\operatorname{Sp}(2 n, R)$. Given a form net of ideals $(\sigma, \Gamma)$ denote by $\operatorname{Ep}(\sigma, \Gamma)$ the subgroup of $\operatorname{Ep}(2 n, R)$ generated by all short and long symplectic transvections $T_{i j}(\xi), T_{i,-i}(\alpha)$, where $i, j \in I, i \neq \pm j, \xi \in \sigma_{i j}$ and $\alpha \in \Gamma_{i}$. The central result of this paper is the following theorem (see Sections 2 and 3 for the definitions omitted here).

Theorem 1. Let $\nu$ be a unitary equivalence relation on the index set I such that $h(\nu) \geq$ $(4,5)$. Let $H$ be a subgroup of $\operatorname{Sp}(2 n, R)$ such that $\operatorname{Ep}(\nu, R) \leq H$. Then there exists a unique exact form net of ideals $(\sigma, \Gamma) \geq[\nu]_{R}$ such that

$$
\operatorname{Ep}(\sigma, \Gamma) \leq H \leq \mathrm{N}_{\mathrm{Sp}(2 n, R)}(\operatorname{Sp}(\sigma, \Gamma)) .
$$

Another important result is that the normalizer $N_{\mathrm{Sp}(2 n, R)}(\operatorname{Sp}(\sigma, \Gamma))$ coincides with the 
transporter

$$
\begin{aligned}
\operatorname{Transp}_{\mathrm{Sp}(2 n, R)}(\operatorname{Ep}(\sigma, \Gamma), & \operatorname{Sp}(\sigma, \Gamma))= \\
& \left\{a \in \operatorname{Sp}(2 n, R) \mid \text { for all } \tau \in \operatorname{Ep}(\sigma, \Gamma) a \tau a^{-1} \in \operatorname{Sp}(\sigma, \Gamma)\right\}
\end{aligned}
$$

in $\operatorname{Sp}(2 n, R)$ from $\operatorname{Ep}(\sigma, \Gamma)$ to $\operatorname{Sp}(\sigma, \Gamma)$ and can be described in terms of congruences.

Theorem 2. Let $\nu$ be a unitary equivalence relation on the index set I such that all the equivalence classes of $\nu$ contain at least 3 elements. Let $(\sigma, \Gamma)$ be a form net of ideals over $R$ such that $[\nu]_{R} \leq(\sigma, \Gamma)$. Then the normalizer $\mathrm{N}_{\mathrm{Sp}(2 n, R)}(\operatorname{Sp}(\sigma, \Gamma))$ coincides with the transporter $\operatorname{Transp}_{\mathrm{Sp}(2 n, R)}(\operatorname{Ep}(\sigma, \Gamma), \operatorname{Sp}(\sigma, \Gamma))$ and consists precisely of all matrices a in $\operatorname{Sp}(2 n, R)$ such that the following three properties hold:

(T1) $a_{i j} \sigma_{j k} a_{k l}^{\prime} \leq \sigma_{i l}$ for all $i, j, k, l \in I$

(T2) $a_{i j}^{2} \sigma_{j k}^{\square} S_{k,-k}\left(a^{-1}\right) \in \Gamma_{i}$ for all $i, j, k \in I$

(T3) $a_{i j}^{2} \Gamma_{j} \leq \Gamma_{i}$ for all $i, j \in I$,

where $\sigma_{j k}^{\natural}$ stands for $\left\{\xi^{2} \mid \xi \in \sigma_{j k}\right\}$.

The rest of this paper is organized as follows. In Sections 2 and 3 we give all the required notation and definitions. In Section 4 we construct the net associated with a subgroup and prove Theorem 2. Sections 5 9 comprise the proof of Theorem 1. We will show that $H \leq \operatorname{Transp}_{\mathrm{Sp}(2 n, R)}(\operatorname{Ep}(\sigma, \Gamma), \operatorname{Sp}(\sigma, \Gamma))$, where $(\sigma, \Gamma)$ is the net associated with $H$. Given a matrix $a \in H$ and an elementary symplectic transvection $T_{s r}(\xi) \in \operatorname{Ep}(\sigma, \Gamma)$ it will be shown that $b=a T_{s r}(\xi) a^{-1} \in \operatorname{Sp}(\sigma, \Gamma)$. By definition of $\operatorname{Sp}(\sigma, \Gamma)$ the inclusion $b \in \operatorname{Sp}(\sigma, \Gamma)$ is equivalent to the following series of inclusions:

$$
T_{i j}\left(b_{i j}\right) \in H \text { for all } i \neq \pm j \quad T_{i,-i}\left(S_{i,-i}(b)\right) \in H \text { for all } i \in I
$$

where $S_{i,-i}(b)=\sum_{j>0} b_{i j} b_{j,-i}^{\prime}$. In order to prove these inclusions we will express the matrices $T_{i j}\left(b_{i j}\right)$ and $T_{i,-i}\left(S_{i,-i}(b)\right)$ as products of elements of $\operatorname{Ep}(\sigma, \Gamma)$ as well as marices $b$ and $b^{-1}$. We shall refer to this procedure as extraction of transvections. First, in Section 5 we introduce a useful abstraction that simplifies loclization based versions of extraction of transvections. In Section [6 we collect the results on extracting transvections from parabolic subgroups. In Sections 7 and 8 we deal with the case of a local ground ring and the Section 9 provides the proof of Theorem 1 for an arbitrary commutative ring using localization and patching.

\section{Notations}

Throughout this paper we will adhere to the following notations and conventions. By ring we will always mean associative unital ring. Given a ring $R$ and a natural number $n$ we will denote by $\mathrm{M}(n, R)$ the full matrix ring of rank $n$ over $R$ and by $\operatorname{GL}(n, R)$ the group of invertible elements of $\mathrm{M}(n, R)$. For any matrix $a$ in $\mathrm{M}(n, R)$ let $a_{i j}$ denote 
the entry of $a$ at the position $(i, j)$ and $a_{i j}^{\prime}$ the corresponding entry of the matrix $a^{-1}$ inverse to $a$. We will denote by $a^{t}$ the matrix transpose of $a$, i.e. the matrix in $\operatorname{M}(n, R)$ such that $\left(a^{t}\right)_{i j}=a_{j i}$. By $a_{i *}$ and $a_{* j}$ we will denote the $i$ 'th row and $j$ 'th column of $a$ respectively. Naturally, $a_{i *}^{\prime}$ and $a_{* j}^{\prime}$ should be read as the $i$ 'th row and $j$ 'th column of $a^{-1}$ respectively. We will also use the notation $\operatorname{diag}\left(\xi_{1}, \ldots, \xi_{n}\right)$ for the diagonal matrix with entries $\xi_{1}, \ldots \xi_{n}$ reading from the top-left corner and $\operatorname{sdiag}\left(\xi_{1}, \ldots, \xi_{n}\right)$ for the skewdiagonal matrix with entries $\xi_{1}, \ldots \xi_{n}$ reading from the top-right corner. When the rank of the matrix ring is clear from the context, we will also denote by diag the diagonal embedding of $R$ into $\mathrm{M}(n, R)$, i.e. diag $: R \rightarrow \mathrm{M}(n, R)$ is a ring homomorphism sending each $\xi \in R$ to the diagonal matrix $\operatorname{diag}(\xi)=\operatorname{diag}(\xi, \ldots, \xi)$. We will denote by $e_{n}$ the unity of the matrix ring $\mathrm{M}(n, R)$. When the rank is clear from the context, we will simply write $e$. The entries of $e$, as an exception from the above convention, will be denoted by $\delta_{i j}$ (Kronecker delta), while $e_{i j}$ will stand for the corresponding standard matrix unit, i.e the matrix in $\mathrm{M}(n, R)$ whose $(i, j)$ 'th entry equals 1 and whose other entries are zero. Given a ring morphism $\varphi: R \rightarrow Q$ we will denote by $\mathrm{M}_{n}(\varphi)=\mathrm{M}(\varphi)$ the induced ring morphism of the matrix rings $\mathrm{M}(n, R)$ and $\mathrm{M}(n, Q)$. If we consider $\mathrm{M}(\varphi)$ as a morphism of the multiplicative monoids of $\mathrm{M}(n, R)$ and $\mathrm{M}(n, Q)$ then its kernel is precisely the set $\mathrm{M}(n, R, \operatorname{ker}(\varphi))=\left\{a \in \mathrm{M}(n, R) \mid a_{i j} \equiv \delta_{i j} \bmod \operatorname{ker}(\varphi)\right.$ for all $\left.i, j\right\}$. Note that $\operatorname{GL}(2 n, R, \operatorname{ker}(\varphi))=\operatorname{GL}(2 n, R) \cap \mathrm{M}(2 n, R, \operatorname{ker}(\varphi))$ is a normal subgroup in $\mathrm{GL}(2 n, R)$.

In our applications it is convenient to index the rows and columns of $2 n \times 2 n$ matrices by the ordered set $I=I_{2 n}=\{1, \ldots, n,-n, \ldots,-1\}$. We equip the poset $I$ with the sign $\operatorname{map} \varepsilon: I \longrightarrow\{ \pm 1\}$, defined by

$$
\varepsilon(i)=\left\{\begin{array}{ll}
+1 & i>0 \\
-1 & i<0
\end{array} .\right.
$$

For the sake of shortening formulas we will also denote $\varepsilon(i)$ by $\varepsilon_{i}$.

Now consider an equivalence relation $\nu$ on the set $I$. If two indices $i$ and $j$ are equivalent under $\nu$, we will write $i \sim^{\nu} j$ or just $i \sim j$ when the equivalence relation is clear from the context. The equivalence class of an index $i$ will be denoted by $\nu(i)$. Call $\nu$ unitary if for any equivalent indices $i$ and $j$ the indices $-i$ and $-j$ are also equivalent. All the equivalence relations mentioned in this paper are unitary and thus we will sometimes omit the word "unitary". The index set $I$ can be decomposed as a disjoint union of equivalence classes:

$$
I=C_{1} \sqcup C_{2} \sqcup \cdots \sqcup C_{t} \text {. }
$$

We introduce a left action of the group $\{ \pm 1\}$ on the set $\mathcal{C l}(\nu)=\left\{C_{1}, \ldots, C_{t}\right\}$ of all equivalence classes $C_{l}$ of $\nu$ by putting: $1 \cdot \nu(i)=\nu(i)$ and $-1 \cdot \nu(i)=\nu(-i)$, for any $i \in I$. Following $\operatorname{Vav08}$ we will call the classes stable under this action self-conjugate (i.e. the classes $C_{l}$ such that for every $i \in C_{l}$ one has also $-i \in C_{l}$ ). Accordingly the non-stable classes will be called non-self-conjugate. We will denote by $h(\nu)$ the ordered pair consisting of the minimum size (as a set) of all self-conjugate equivalence classes 
of $\nu$ and the minimum size (also as a set) of all non-self-conjugate equivalence classes of $\nu$. Note, that an arbitrary equivalence relation does not necessary have equivalent classes of both types; therefore $h(\nu)$ is an element in $\mathbb{N} \cup\{\infty\} \times \mathbb{N} \cup\{\infty\}$. We will always view $\mathbb{N} \cup\{\infty\} \times \mathbb{N} \cup\{\infty\}$ as a partially ordered set with the product order, i.e. $\left(a_{1}, b_{1}\right) \leq\left(a_{2}, b_{2}\right)$ if and only if $a_{1} \leq a_{2}$ and $b_{1} \leq b_{2}$.

We call a $k$-tuple $\left(i_{1}, \ldots, i_{k}\right)$ of indices in $I$ a $\mathrm{C}$-type base $k$-tuple [of indices] if for each $1 \leq r \neq s \leq k$, we have $i_{r} \neq \pm i_{s}$ and $i_{r} \sim i_{s} \sim-i_{s} \sim-i_{r}$. Similarly, we call a $k$-tuple $\left(i_{1}, \ldots, i_{k}\right)$ of indices in $I$ an A-type base $k$-tuple [of indices] if for each $1 \leq r \neq s \leq k$, we have $i_{r} \neq \pm i_{s}$ and $i_{r} \sim i_{s}$. The condition $h(\nu) \geq(a, b)$ is equivalent to the condition that every index $i \in I$ can be included in either an A-type base $b$-tuple, or a C-type base $\left\lceil\frac{a}{2}\right\rceil$-tuple. This simple observation will be used repeatedly without specific mention in the rest of the paper.

Another convention concerns the elements of a localization of a ring. Let $R$ be a unital associative ring and $S$ be a multiplicative set in $R$. Let $S^{-1} R$ denote the localization of $R$ at $S$. Let $F: R \rightarrow S^{-1} R$ be the localization morphism. Let $\xi \in R$ and $s \in S$. By the fraction $\frac{\xi}{s}$ we will always denote the element $F(\xi) \cdot F(s)^{-1}$ of $S^{-1} R$ in contrast to $\xi s^{-1}$ which refers to an element of $R$ and only makes sense if $s \in R^{*}$. For example $\frac{\xi}{1}$ is synonymic to $F(\xi)$.

Finally, by angular brackets $\langle\cdot\rangle$ we will denote the subgroups and ideals defined in terms of generators. The rest of the notations are standard for the field of this research.

\section{Preliminaries}

Symplectic group Let $R$ be a commutative ring. Fix a natural number $n$. Let $\operatorname{Sp}(2 n, R)$ denote the classical symplectic group of rank $2 n$. It is known that a matrix $a$ in $\operatorname{GL}(2 n, R)$ belongs to $\operatorname{Sp}(2 n, R)$ if and only if the equality

$$
a_{i j}^{\prime}=\varepsilon_{i} \varepsilon_{j} a_{-j,-i}
$$

holds for all possible indices. In future, this property will be used without reference.

Given an element $\xi \in R$ and an index $i \in I$ we will call the matrix

$$
T_{i,-i}(\xi)=e+\xi e_{i,-i}
$$

the [elementary] long [symplectic] transvection. Given an additional index $j \neq \pm i$ we will call the matrix

$$
T_{i j}(\xi)=e+\xi e_{i j}-\varepsilon_{i} \varepsilon_{j} \xi e_{-j,-i}
$$

the [elementary] short [symplectic] transvection.

It's a well known fact, that all the long and short elementary symplectic transvections are containd in $\operatorname{Sp}(2 n, R)$ and satisfy the following relations known as the Steinberg relations for all $\xi, \zeta$ in $R$ :

(R1) $T_{i j}(\xi)=T_{-j,-i}\left(-\varepsilon_{i} \varepsilon_{j} \xi\right)$ for all $i \neq \pm j$

(R2) $T_{i j}(\xi) T_{i j}(\zeta)=T_{i j}(\xi+\zeta)$ for all $i \neq j$ 
(R3) $\left[T_{i j}(\xi), T_{h k}(\zeta)\right]=e$ for all $h \neq j,-i$ and $k \neq i,-j$

(R4) $\left[T_{i j}(\xi), T_{j h}(\zeta)\right]=T_{i h}(\xi \zeta)$ for all $i, h \neq \pm j$ and $i \neq \pm h$

(R5) $\left[T_{i j}(\xi), T_{j,-i}(\zeta)\right]=T_{i,-i}(2 \xi \zeta)$ for all $i$

(R6) $\left[T_{i,-i}(\xi), T_{-i, j}(\zeta)\right]=T_{i j}(\xi \zeta) T_{-j, j}\left(\varepsilon_{i} \varepsilon_{j} \xi \zeta^{2}\right)$ for all $i \neq \pm j$.

In future we will occasionally use these relations without a reference.

For any matrix $g$ in $\operatorname{Sp}(2 n, R)$, any short symplectic transvection $T_{s r}(\xi)$ and any long symplectic transvection $T_{s,-s}(\zeta)$, we call the matrices ${ }^{g} T_{s r}(\xi)=g T_{s r}(\xi) g^{-1}$ and ${ }^{g} T_{s,-s}(\zeta)=g T_{s,-s}(\zeta) g^{-1}$ [elementary] short and long root elements respectively.

Equivalence relations and block-diagonal subgroups Given a unitary equivalence relation $\nu$ on the index set $I$ we will call the subgroup

$$
\operatorname{Ep}(\nu)=\operatorname{Ep}(\nu, R)=\left\langle T_{i,-i}(\xi), T_{j k}(\xi) \mid i \sim-i, j \sim k, j \neq \pm k, \xi \in R\right\rangle
$$

the elementary block-diagonal subgroup of type $\nu$ in $\operatorname{Sp}(2 n, R)$. Note that $\operatorname{Ep}(\nu, R)$ does not necessarily consist of block-diagonal matrices. It can only be the case if all (or at least all but one of) the equivalence classes of $\nu$ are non-self-conjugate. However, these groups behave like block-diagonal matrices because for each such group $\operatorname{Ep}(\nu, R)$ there exists a permutation matrix $B$ in $\operatorname{GL}(2 n, R)$, but not necessarily in $\operatorname{Sp}(2 n, R)$, such that $B \cdot \operatorname{Ep}(\nu, R) \cdot B^{-1}$ is block-diagonal in the usual sense.

From the point of view of Chevalley groups, the elementary block-diagonal subgroup is precisely the elementary subsystem subgroup. Namely, let $C_{1},-C_{1}, \ldots C_{s},-C_{s}$ be all the non-self-conjugate classes, and $C_{s+1}, \ldots, C_{t}$ be the self-conjugate ones. Set $n_{i}=\left|C_{i}\right|$ for $1 \leq i \leq s$ and $l_{i}=\left|C_{i}\right| / 2$ for $s+1 \leq i \leq t$. Then $n_{1}+\cdots+n_{s}+l_{s+1}+\cdots+l_{t}=n$ and

$$
\operatorname{Ep}(\nu, R) \approx \mathrm{E}\left(n_{1}, R\right) \times \cdots \times \mathrm{E}\left(n_{s}, R\right) \times \operatorname{Ep}\left(2 l_{s+1}, R\right) \times \cdots \times \operatorname{Ep}\left(2 l_{t}, R\right),
$$

where $\mathrm{E}\left(n_{i}, R\right)$ denotes the usual elementary subgroup of $\operatorname{GL}\left(n_{i}, R\right)$ appearing in the hyperbolic embedding and the product is meant as a product of linear groups. From the viewpoint of algebraic groups this is exactly the elementary Chevalley group of type $\Delta$, where

$$
\Delta=A_{n_{1}-1}+\cdots+A_{n_{s}-1}+C_{l_{s+1}}+\cdots+C_{l_{t}} .
$$

The reader is referred to [Vav08] and the references therein for further details.

This analogy allows the following geometric interpretation of concepts of A-type and C-type base tuples. By choosing a unitary equivalence relation $\nu$ we fix a subsystem $\Delta \leq C_{n}$ consisting of all roots $\alpha_{i j} \in C_{n}$ where $i \sim^{\nu} j$. Irreducible components of $\Delta$ are in one to one correspondence with equivalence classes of $\nu$, namely the components of type $C_{l}$ correspond to self-conjugate equivalence classes and the components of type $A_{l}$ correspond to pairs of non-self-conjugate classes. Then an A- or C- type base $k$ tuple $\left(i_{1}, \ldots, i_{k}\right)$ provides us with a root subsystem in $\Delta$ of type $A_{k-1}$ or $C_{k}$ respectively, namely $\left\langle\alpha_{i_{1}, i_{2}}, \ldots, \alpha_{i_{k-1}, i_{k}}\right\rangle$ or $\left\langle\alpha_{i_{1}, i_{2}}, \ldots, \alpha_{i_{k-1}, i_{k}}, \alpha_{i_{k}}\right\rangle$, respectively. Moreover, both generating sets above can be chosen as systems (or bases) of simple roots in the subsystems they generate. 
Form nets of ideals and corresponding groups Consider a square array $\sigma=\left(\sigma_{i j}\right)_{i, j \in I}$ of $(2 n)^{2}$ ideals of the ring $R$. We will call it a net of ideals over $R$ if for any indices $i, j$ and $k$, we have the following inclusions:

$$
\sigma_{i k} \sigma_{k j} \leq \sigma_{i j}
$$

A net of ideals $\sigma$ is called unitary, if $\sigma_{i j}=\sigma_{-j,-i}$ for each $i$ and $j$. We will call $\sigma$ a D-net, if $\sigma_{i i}=R$ for every $i$ in $I$. Equip the net of ideals $\sigma$ with $2 n$ additive subgroups $\Gamma=\left(\Gamma_{i}\right)_{i \in I}$ of $R$ such that for any indices $i, j \in I$ the following inclusions hold:

1. $2 \sigma_{i,-i} \leq \Gamma_{i} \leq \sigma_{i,-i}$

2. $\sigma_{i j}^{\sqrt[2]{2}} \Gamma_{j} \leq \Gamma_{i}$,

where $2 \sigma_{i,-i}=\left\{2 \alpha \mid \alpha \in \sigma_{i,-i}\right\}$ and $\sigma_{i j}^{2}=\left\{\xi^{2} \mid \xi \in \sigma_{i j}\right\}$. In this situation $\Gamma$ is called $a$ column of form parameters for $\sigma$ and the pair $(\sigma, \Gamma)$ a form net of ideals [over $R]$. It is the analogue for nets of ideals of the concept of form ideal of Bak [Bak69] for form rings. A form net of ideals $(\sigma, \Gamma)$ is said to be exact if for any index $i$ the equality

$$
\sigma_{i,-i}=\sum_{k \neq \pm i} \sigma_{i k} \sigma_{k,-i}+\left\langle\Gamma_{i}\right\rangle
$$

holds. From now on we only deal with unitary exact form D-nets of ideals.

Introduce a partial ordering on the set of all form nets of ideals over $R$ by setting $\left(\sigma^{\prime}, \Gamma^{\prime}\right) \leq\left(\sigma^{\prime \prime}, \Gamma^{\prime \prime}\right)$ whenever for all $i, j \in I$ the inclusions $\sigma_{i j}^{\prime} \leq \sigma_{i j}^{\prime \prime}$ and $\Gamma_{i}^{\prime} \leq \Gamma_{i}^{\prime \prime}$ hold. As a matter of convenience, given an element $\xi \in R$ and indices $s$ and $r$ we will write " $\xi \in(\sigma, \Gamma)_{s r}$ " instead of " $\xi \in \sigma_{s r}$ if $r \neq-s$ and $\xi \in \Gamma_{s}$ otherwise".

We can associate two kinds of subgroups of $\operatorname{Sp}(2 n, R)$ to each form net of ideals $(\sigma, \Gamma)$ over $R$. We call the subgroup

$$
\operatorname{Ep}(\sigma, \Gamma)=\left\langle T_{i j}(\xi), T_{i,-i}(\alpha) \mid i \neq \pm j, \xi \in \sigma_{i j}, \alpha \in \Gamma_{i}\right\rangle
$$

the elementary form net subgroup of level $(\sigma, \Gamma)$. We call the above generators of $\operatorname{Ep}(\sigma, \Gamma)$ the short and long $(\sigma, \Gamma)$-elementary symplectic transvections, respectively. Note that any unitary equivalence relation $\nu$ on the set of indices $I$ defines a form net

$$
[\nu]_{R}=\left(\sigma_{\nu}, \Gamma_{\nu}\right)
$$

where

$$
\left(\sigma_{\nu}\right)_{i j}=\left\{\begin{array}{ll}
R, & \text { if } i \sim^{\nu} j \\
0, & \text { if } i \nsim^{\nu} j
\end{array} \quad\left(\Gamma_{\nu}\right)_{i}=\left\{\begin{array}{ll}
R, & \text { if } i \sim^{\nu}-i \\
0 & i \nsim^{\nu}-i
\end{array} .\right.\right.
$$

This is clearly a D-net. Thus the elementary block-diagonal subgroup $\operatorname{Ep}(\nu, R)=$ $\operatorname{Ep}\left(\sigma_{\nu}, \Gamma_{\nu}\right)$ is a special case of an elementary form net subgroup. We will call a form net of ideals $(\sigma, \Gamma)$ major [with respect to $\nu]$ if $[\nu]_{R} \leq(\sigma, \Gamma)$. The notion of elementary form net subgroup is a generalization of that of relative elementary subgroup of even 
unitary groups introduced in Bak [Bak81, p. 66]. The concept of the relative principal congruence subgroups therein is generalized as follows. We will call the subgroup

$$
\operatorname{Sp}(\sigma, \Gamma)=\left\{g \in \operatorname{Sp}(2 n, R) \mid \forall i, j \in I g_{i j} \in \sigma_{i j}, S_{i,-i}(g) \in \Gamma_{i}\right\}
$$

the form net subgroup of level $(\sigma, \Gamma)$, where

$$
S_{i,-i}(g)=\sum_{j>0} g_{i j} g_{j,-i}^{\prime}
$$

is the so called length of the row $g_{i *}$. The element $S_{i,-i}(g)$ is clearly in $\sigma_{i,-i}$, by definition of a net of ideals, and insisting that $S_{i,-i}(g) \in \Gamma_{i} \leq \sigma_{i,-i}$ is a further restrinction on $g$. The word "length" was introduced by You in You12.

In the situation when $\Gamma_{i}=R$ for all $i$ the form net subgroup coincides with the regular net subgroup $\operatorname{Sp}(\sigma)=\left\{g \in \operatorname{Sp}(2 n, R) \mid \forall i, j \in I g_{i j} \in \sigma_{i j}\right\}$.

Next we compute the lengths of rows of certain products of matrices. An immediate reason to do so is to prove that the above defined set $\operatorname{Sp}(\sigma, \Gamma)$ is indeed a group. Besides, these computations are used repeatedly in most of the proofs in this paper. Despite of the importance of the proposition below and it's corollaries, it's proof is quite routine and thus is omitted (cf. Shc15] for the proof).

Proposition 3.1. Let $a$ and $b$ be two matrices in $\operatorname{Sp}(2 n, R)$. Then

$$
\begin{aligned}
S_{i,-i}(a b)= & S_{i,-i}(a)+\sum_{k} a_{i k} S_{k,-k}(b) a_{-k,-i}^{\prime}- \\
& -2 \sum_{j, k, l>0} a_{i, l} b_{l,-j} b_{-j, k}^{\prime} a_{k,-i}^{\prime}- \\
& -2 \sum_{j, k>0} \sum_{l>k}\left(a_{i,-k} b_{-k,-j} b_{-j, l}^{\prime} a_{l,-i}^{\prime}+a_{i k} b_{k,-j} b_{-j,-l}^{\prime} a_{-l,-i}^{\prime}\right) .
\end{aligned}
$$

Corollary 3.2. Let $(\sigma, \Gamma)$ be a form net of ideals over $R$. Suppose $a, b \in \operatorname{Sp}(\sigma)$, then

$$
S_{i,-i}(a b) \equiv S_{i,-i}(a)+\sum_{k} a_{i k}^{2} S_{k,-k}(b) \quad \bmod \Gamma_{i}
$$

In particular, the form net subgroup $\operatorname{Sp}(\sigma, \Gamma)$ is indeed a group.

Proof. Clearly $e \in \operatorname{Sp}(\sigma, \Gamma)$. Next the congruences (1) together with the condition $\sigma_{i k}^{[2]} \Gamma_{k} \leq \Gamma_{i}$ show that $\operatorname{Sp}(\sigma, \Gamma)$ is closed under taking products. Let $a \in \operatorname{Sp}(\sigma, \Gamma)$. By (1) we get that

$$
0=S_{i,-i}(e)=S_{i,-i}\left(a^{-1} a\right) \equiv S_{i,-i}\left(a^{-1}\right)+\sum_{k}\left(a_{i k}^{\prime}\right)^{2} S_{k,-k}(a) \equiv S_{i,-i}\left(a^{-1}\right) \quad \bmod \Gamma_{i} .
$$

Therefore $a^{-1} \in \operatorname{Sp}(\sigma, \Gamma)$ and $\operatorname{Sp}(\sigma, \Gamma)$ is a group. 
The following two corollaries allow us to compute the lengths of rows in products of matrices in $\operatorname{Sp}(\sigma)$ and short $(\sigma, \Gamma)$-elementary symplectic transvection as well as lengths of rows of some root elements.

Corollary 3.3. Let $a \in \operatorname{Sp}(\sigma)$ and $T_{p q}(\xi)$ be a short elementary symplectic transvection in $\operatorname{Ep}(\sigma, \Gamma)$. Then

$$
S_{i,-i}\left(T_{p q}(\xi) a\right) \equiv\left\{\begin{array}{lll}
S_{i,-i}(a) & \text { if } i \neq p,-q \\
S_{p,-p}(a)+\xi^{2} S_{q,-q}(a) & \text { if } i=p \\
S_{-q, q}(a)+\xi^{2} S_{-p, p}(a) & \text { if } i=-q &
\end{array} \quad \bmod \Gamma_{i}\right.
$$

and for all $i \in I$

$$
S_{i,-i}\left(a T_{p q}(\xi)\right) \equiv S_{i,-i}(a) \bmod \Gamma_{i}
$$

Corollary 3.4. Let $a \in \operatorname{Sp}(\sigma), T_{s r}(\xi), T_{s t}(\zeta)$ be short elementary symplectic transvections in $\operatorname{Ep}(\sigma)$ and $s \neq \pm r, \pm t$ and $r \neq \pm t$. Then

$$
\begin{aligned}
S_{i,-i}\left(a T_{s r}(\xi) T_{s t}(\zeta) a^{-1}\right) & \equiv a_{i s}^{2} \zeta^{2} S_{t,-t}\left(a^{-1}\right)+a_{i s}^{2} \xi^{2} S_{r,-r}\left(a^{-1}\right)+ \\
& +a_{i,-t}^{2} \zeta^{2} S_{-s, s}\left(a^{-1}\right)+a_{i,-r}^{2} \xi^{2} S_{-s, s}\left(a^{-1}\right) \quad \bmod \Gamma_{i} .
\end{aligned}
$$

In particular if $\zeta=0$ then $T_{s t}(\zeta)=e$ and

$$
S_{i,-i}\left(a T_{s r}(\xi) a^{-1}\right) \equiv a_{i s}^{2} \xi^{2} S_{r,-r}\left(a^{-1}\right)+a_{i,-r}^{2} \xi^{2} S_{-s, s}\left(a^{-1}\right) \quad \bmod \Gamma_{i} .
$$

We finish this section with two technical results which will be used repeatedly and without reference in proofs throughout the paper. The first one shows that major form nets of ideals are partitioned into blocks in which all ideals are equal and all form parameters are equal. The second one allows simplifying reasoning dealing with caseby-case analysis of small equivalence classes. The proofs of both results can be checked straightforwardly and are left to the reader.

Proposition 3.5. Let $(\sigma, \Gamma)$ be a form net of ideal such that $\operatorname{Ep}(\nu, R) \leq \operatorname{Ep}(\sigma, \Gamma)$, with $h(\nu) \geq(4,3)$. Then for any indices $i, j, k, l$ such that $k \sim i, l \sim j$, we have:

1. $\sigma_{k j}=\sigma_{i j}=\sigma_{i l}$

2. $\Gamma_{i}=\Gamma_{k}$.

Proposition 3.6. Let $\nu$ be a unitary equivalence relation on the index set I such that $h(\nu) \geq(4,3)$. Let $i, j$ be two indices in I such that $i \neq j$. Then one of the following holds:

1. $\nu(i)=\{i,-i, j,-j\}$

2. There exists an index $k$ in $I$ such that $k \neq \pm i, \pm j$ and $k \sim^{\nu} i$. 


\section{Form net associated with a subgroup and the description of the transporter}

Form net of ideals associated with a subgroup. Let $\nu$ be a unitary equivalence relation on the index set $I=\{1, \ldots, n,-n, \ldots,-1\}$ such that $h(\nu) \geq(4,3)$. Let $H$ be a subgroup of $\operatorname{Sp}(2 n, R)$ such that $\operatorname{Ep}(\nu, R) \leq H$. An exact form net of ideals $(\sigma, \Gamma)$ is called the form net [of ideals] associated with $H$ if $\operatorname{Ep}(\sigma, \Gamma) \leq H$ and if for any exact form net of ideals $\left(\sigma^{\prime}, \Gamma^{\prime}\right)$ such that $\operatorname{Ep}\left(\sigma^{\prime}, \Gamma^{\prime}\right) \leq H$, it follows that $\left(\sigma^{\prime}, \Gamma^{\prime}\right) \leq(\sigma, \Gamma)$. Clearly, if $(\sigma, \Gamma)$ exists then it is unique. The next lemma shows that $(\sigma, \Gamma)$ exists.

Lemma 4.1. Let $\nu$ be a unitary equivalence relation on $I$ such that $h(\nu) \geq(4,3)$ and $H$ a subgroup of $\operatorname{Sp}(2 n, R)$ that contains the subgroup $\operatorname{Ep}(\nu, R)$. For each $i \neq \pm j \in I$ set

$$
\begin{gathered}
\sigma_{i j}=\left\{\xi \in R \mid T_{i j}(\xi) \in H\right\}, \quad \Gamma_{i}=\left\{\alpha \in R \mid T_{i,-i}(\alpha) \in H\right\}, \quad \sigma_{i i}=R, \\
\sigma_{i,-i}=\sum_{j \neq \pm i} \sigma_{i j} \sigma_{j,-i}+\left\langle\Gamma_{i}\right\rangle .
\end{gathered}
$$

Then $(\sigma, \Gamma)$ is the form net of ideals associated with $H$.

We won't prove this lemma in the present form. In section 5 we will prove a slightly stronger version of this result, Proposition 5.2. For the sake of simplicty, the proof Proposition 5.2 relies on Lemma 4.1. This does not create a loop in theory, as we can almost literaly repeat the proof of Proposition 5.2 for Lemma 4.1. This is done explicitly in Shc15, Chapter 1, Lemma 1.2.1].

Description of the transporter. The rest of this section is devoted to the proof of Theorem 2. The following proposition allows to compute lengths of rows of conjugates of an arbitrary matrix by a matrix satisfying property (T1) of Theorem 2 ,

Proposition 4.2. Let $(\sigma, \Gamma)$ be an exact form net of ideals. Suppose a $\in \operatorname{Sp}(2 n, R)$ satisfies the condition

$$
a_{i j} \sigma_{j k} a_{k l}^{\prime} \leq \sigma_{i l}
$$

for all $i, j, k, l \in I$. Then for any matrix $g \in \operatorname{Sp}(\sigma, \Gamma)$ and any $i \in I$ the following congruence holds:

$$
S_{i,-i}\left(a g a^{-1}\right) \equiv \sum_{k \in I} a_{i k}^{2}\left(S_{k,-k}(g)+S_{k,-k}\left(a^{-1}\right)+\sum_{t \in I} g_{k t}^{2} S_{t,-t}\left(a^{-1}\right)\right) \quad \bmod \Gamma_{i} .
$$

Proof. By Proposition 3.1 we get

$$
\begin{aligned}
S_{i,-i}\left(a g a^{-1}\right)= & S_{i,-i}(a)+\sum_{k \in I} \varepsilon_{i} \varepsilon_{k} a_{i k}^{2} S_{k,-k}\left(g a^{-1}\right) \\
& -2 \sum_{j, k, l>0} a_{i l}\left(g a^{-1}\right)_{l,-j}\left(a g^{-1}\right)_{-j, k} a_{k,-i}^{\prime} \\
& -2 \sum_{j, k>0} \sum_{l>k}\left(a_{i,-k}\left(g a^{-1}\right)_{-k,-j}\left(a g^{-1}\right)_{-j, l} a_{l,-i}^{\prime}+\right. \\
& \left.a_{i k}\left(g a^{-1}\right)_{k,-j}\left(a g^{-1}\right)_{-j,-l} a_{-l,-i}^{\prime}\right) .
\end{aligned}
$$


Consider an individual summand of the second big sum above. By the assumption that $a_{i j} \sigma_{j k} a_{k l}^{\prime} \leq \sigma_{i l}$ we get

$$
a_{i l}\left(g a^{-1}\right)_{l,-j}\left(a g^{-1}\right)_{-j, k} a_{k,-i}^{\prime}=\sum_{p, q \in I}\left(a_{i l} g_{l p} a_{p,-j}^{\prime}\right)\left(a_{-j, q} g_{q k}^{\prime} a_{k,-i}^{\prime}\right) \leq \sigma_{i,-j} \sigma_{-j,-i} \leq \sigma_{i,-i}
$$

and therefore the doubled second big sum in (2) is contained in $2 \sigma_{i,-i} \leq \Gamma_{i}$. Applying the same principle to the last summand in (2) we get the inclusion

$$
\left.a_{i,-k}\left(g a^{-1}\right)_{-k,-j}\left(a g^{-1}\right)_{-j, l} a_{l,-i}^{\prime}+a_{i k}\left(g a^{-1}\right)_{k,-j}\left(a g^{-1}\right)_{-j,-l} a_{-l,-i}^{\prime}\right) \in \sigma_{i,-i} .
$$

Combining (21), (3) and (41) we get

$$
S_{i,-i}\left(a g a^{-1}\right) \equiv S_{i,-i}(a)+\sum_{k \in I} \varepsilon_{i} \varepsilon_{k} a_{i k}^{2} S_{k,-k}\left(g a^{-1}\right) \quad \bmod \Gamma_{i} .
$$

Expand (5) further using Proposition 3.1.

$$
\begin{aligned}
S_{i,-i}\left(a g a^{-1}\right) \equiv & S_{i,-i}(a)+\sum_{k \in I} \varepsilon_{i} \varepsilon_{k} a_{i k}^{2} S_{k,-k}\left(g a^{-1}\right) \\
\equiv & S_{i,-i}(a)+\sum_{k \in I} \varepsilon_{i} \varepsilon_{k} a_{i k}^{2}\left(S_{k,-k}(g)+\sum_{t \in I} \varepsilon_{k} \varepsilon_{t} g_{k t}^{2} S_{t,-t}\left(a^{-1}\right)\right. \\
& -2 \sum_{j, t, l>0} g_{k l} a_{l,-j}^{\prime} a_{-j, t} g_{t,-k}^{\prime} \\
& \left.-2 \sum_{j, t>0} \sum_{l>t}\left(g_{k,-t} a_{-t,-j}^{\prime} a_{-j, l} g_{l,-k}^{\prime}+g_{i t} a_{t,-j}^{\prime} a_{-j,-l} g_{-l,-l}^{\prime}\right)\right) \\
\equiv & S_{i,-i}(a)+\sum_{k \in I} \varepsilon_{i} \varepsilon_{k} a_{i k}^{2}\left(S_{k,-k}(g)+\sum_{t \in I} \varepsilon_{k} \varepsilon_{t} g_{k t}^{2} S_{t,-t}\left(a^{-1}\right)\right) \\
& -2 \sum_{j, t, l>0} a_{i k} g_{k l} a_{l,-j}^{\prime} a_{-j, t} g_{t,-k}^{\prime} a_{-k,-i}^{\prime} \\
& -2 \sum_{j, t>0} \sum_{l>t}\left(a_{i k} g_{k,-t} a_{-t,-j}^{\prime} a_{-j, l} g_{l,-k}^{\prime} a_{-k,-i}^{\prime}\right. \\
& \left.+a_{i k} g_{i t} a_{t,-j}^{\prime} a_{-j,-l} g_{-l,-l}^{\prime} a_{-k,-i}^{\prime}\right)
\end{aligned}
$$

$\bmod \Gamma_{i}$.

Using the same trick as before we may conclude that both doubled terms of (6) are contained in $2 \sigma_{i,-i} \leq \Gamma_{i}$. Summing up, we get the congruence

$$
S_{i,-i}\left(a g a^{-1}\right) \equiv S_{i,-i}(a)+\sum_{k \in I} \varepsilon_{i} \varepsilon_{k} a_{i k}^{2}\left(S_{k,-k}(g)+\sum_{t \in I} \varepsilon_{k} \varepsilon_{t} g_{k t}^{2} S_{t,-t}\left(a^{-1}\right)\right) \quad \bmod \Gamma_{i} .
$$


Finally it's easy to see that

$$
\varepsilon_{i} \varepsilon_{k} a_{i k}^{2} S_{k,-k}(g)=\sum_{l>0, p \in I}\left(a_{i k} g_{k l} a_{l p}^{\prime}\right)\left(a_{p l} g_{l,-k}^{\prime} a_{-k,-i}^{\prime}\right) \in \sigma_{i,-i}
$$

and

$$
\varepsilon_{i} \varepsilon_{k} \varepsilon_{k} \varepsilon_{t} a_{i k}^{2} g_{k t}^{2} S_{t,-t}\left(a^{-1}\right)=\sum_{l>0}\left(a_{i k} g_{k t} a_{t l}^{\prime}\right)\left(a_{l,-t}^{\prime} g_{-t,-k}^{\prime} a_{-k,-i}^{\prime}\right) \in \sigma_{i,-i} .
$$

Therefore the choice of signs in (7) is insignificant and we can rewrite (7) as follows

$$
S_{i,-i}\left(a g a^{-1}\right) \equiv S_{i,-i}(a)+\sum_{k \in I} a_{i k}^{2}\left(S_{k,-k}(g)+\sum_{t \in I} g_{k t}^{2} S_{t,-t}\left(a^{-1}\right)\right) \quad \bmod \Gamma_{i} .
$$

It' clear that $S_{i,-i}(e)=0$ for all $i$. Rewrite the formula (8) for $g=e$. As $S_{i,-i}(a)=$ $\sum_{j>0} a_{i j} \delta_{j j} a_{j,-i}^{\prime} \leq \sigma_{i,-i}$, we can also change the sign at the first term:

$$
0=S_{i,-i}\left(a \cdot a^{-1}\right) \equiv-S_{i,-i}(a)+\sum_{k \in I} a_{i k}^{2} S_{k,-k}\left(a^{-1}\right) \quad \bmod \Gamma_{i}
$$

Finally, adding (9) to (8) we get the required inclusion

$$
S_{i,-i}\left(a g a^{-1}\right) \equiv \sum_{k \in I} a_{i k}^{2}\left(S_{k,-k}(g)+S_{k,-k}\left(a^{-1}\right)+\sum_{t \in I} g_{k t}^{2} S_{t,-t}\left(a^{-1}\right)\right) \quad \bmod \Gamma_{i} .
$$

This completes the proof.

Proof of Theorem 2. Denote by $N$ the set of all matrices in $\operatorname{Sp}(2 n, R)$ satisfying the conditions (T1) - (T3). It's easy to see that $N \leq \mathrm{N}_{\mathrm{Sp}(2 n, R)}(\operatorname{Sp}(\sigma, \Gamma))$. Indeed, pick any $g \in \operatorname{Sp}(\sigma, \Gamma)$ and any $a \in N$. Then condition (T1) guarantees that

$$
\left(a g a^{-1}\right)_{i j}=\sum_{p, q \in I} a_{i p} g_{p q} a_{q j}^{\prime} \leq \sum_{p, q \in I} a_{i p} \sigma_{p q} a_{q j}^{\prime} \leq \sigma_{i j}
$$

for all $i, j \in I$. Now applying Proposition 4.2 we get

$$
S_{i,-i}\left(a g a^{-1}\right) \equiv \sum_{k \in I}\left(a_{i k}^{2} S_{k,-k}(g)+a_{i k}^{2} S_{k,-k}\left(a^{-1}\right)+\sum_{t \in I} a_{i k}^{2} g_{k t}^{2} S_{t,-t}\left(a^{-1}\right)\right) \quad \bmod \Gamma_{i} .
$$

Observe that by condition (T3) it follows that $a_{i k}^{2} S_{k,-k}\left(g^{-1}\right) \in a_{i k}^{2} \Gamma_{k} \leq \Gamma_{i}$. Next, by condition (T2) we get $a_{i k}^{2} g_{k t}^{2} S_{t,-t}\left(a^{-1}\right) \in a_{i k}^{2} \sigma_{k t}^{2} S_{t,-t}\left(a^{-1}\right) \leq \Gamma_{i}$ and $a_{i k}^{2} S_{k,-k}\left(a^{-1}\right)=a_{i k}^{2}$. $1^{2} \cdot S_{k,-k}\left(a^{-1}\right) \in a_{i k}^{2} \sigma_{k k}^{2} S_{k,-k}\left(a^{-1}\right) \leq \Gamma_{i}$. Therefore, $\left.S_{i,-i}\left(a g a^{-1}\right)\right) \in \Gamma_{i}$ for all $i$. It follows that $a g a^{-1} \in \operatorname{Sp}(\sigma, \Gamma)$ and thus $a \in \mathrm{N}_{\mathrm{Sp}(2 n, R)}(\operatorname{Sp}(\sigma, \Gamma))$.

The proof of the inclusion $\operatorname{Transp}_{\operatorname{Sp}(2 n, R)}(\operatorname{Ep}(\sigma, \Gamma), \operatorname{Sp}(\sigma, \Gamma)) \leq N$ is slightly trickier. Consider an arbitrary matrix $a$ in $\operatorname{Transp}_{\operatorname{Sp}(2 n, R)}(\operatorname{Ep}(\sigma, \Gamma), \operatorname{Sp}(\sigma))$ and a short $(\sigma, \Gamma)$ elementary transvection $T_{r s}(\xi)$. By definition of transporter we get

$$
\delta_{i j}+a_{i r} \xi a_{s j}^{\prime}-\varepsilon(r) \varepsilon(s) a_{i,-s} \xi a_{-r, j}^{\prime}=\left({ }^{a} T_{r s}(\xi)\right)_{i j} \in \sigma_{i j} .
$$


Now given two short $(\sigma, \Gamma)$-elementary transvections $T_{r s}(\xi)$ and $T_{s t}(\zeta)$ such that $r \neq \pm t$ we get by a straightforward computation

$$
\delta_{i j}+a_{i r} \xi \zeta a_{t j}^{\prime}=\left(a T_{r s}(\xi) T_{s t}(\zeta) a^{-1}\right)_{i j}-\left(a T_{r s}(\xi) a^{-1}\right)_{i j}-\left(a T_{s t}(\zeta) a^{-1}\right)_{i j}+\delta_{i j} .
$$

And therefore using (10) we get the inclusions $a_{i r} \sigma_{r s} \sigma_{s r} a_{t j}^{\prime} \leq \sigma_{i j}$ for all $i, j, s, r, t \in I$ such that $s \neq \pm r, \pm t$ and $r \neq \pm t$.

Next for a long $(\sigma, \Gamma)$-elementary transvection $T_{s,-s}(\alpha)$ we get

$$
\delta_{i j}+a_{i s} \alpha a_{-s, j}^{\prime}=\left({ }^{a} T_{s,-s}(\alpha)\right)_{i j} \in \sigma_{i j} .
$$

Finally for $r, s \in I$ such that $r \neq \pm s$ we get

$$
\delta_{i j}+a_{i r} \xi \alpha a_{-s, j}^{\prime}=\left(a T_{r s}(\xi) T_{s,-s}(\alpha) a^{-1}\right)_{i j}-\left(a T_{r s}(\xi) a^{-1}\right)_{i j}-\left(a T_{s,-s}(\alpha) a^{-1}\right)_{i j}+\delta_{i j}
$$

and therefore by (12) we get the inclusions $a_{i r} \sigma_{r s} \Gamma_{s,-s} a_{r j}^{\prime} \leq \sigma_{i j}$ for all $i, j \in I$ and all $r, s \in I$ such that $s \neq \pm r$.

Now let $r$ and $t$ be two indices such that $r \neq \pm t$. Then either $\nu(r)=\{ \pm r, \pm t\}$, or there exists an index $s \sim t$ such that $s \neq \pm r, \pm t$. In the former case using (13) we get

$$
a_{i r} \sigma_{r t} a_{t j}^{\prime}=a_{i r} R \sigma_{r t} a_{t j}^{\prime}=a_{i r} \sigma_{r,-t} \Gamma_{-t, t} a_{t j}^{\prime} \leq \sigma_{i j}
$$

for all $i, j \in I$. In the latter case using (11) we get

$$
a_{i r} \sigma_{r t} a_{t j}^{\prime}=a_{i r} \sigma_{r t} R a_{t j}^{\prime}=a_{i r} \sigma_{r s} \sigma_{s t} a_{t j}^{\prime} \leq \sigma_{i j}
$$

for all $i, j \in I$.

Now assume $t=r$. Then there exists an index $s \sim r$ such that $s \neq \pm r$ and using the fact that $\sum_{l \in I} a_{s l}^{\prime} a_{l s}=1$ we get

$$
a_{i r} \sigma_{r r} a_{r j}^{\prime}=\sum_{l \in I}\left(a_{i r} \sigma_{r s} a_{s l}^{\prime}\right)\left(a_{l s} \sigma_{s r} a_{r j}^{\prime}\right) \leq \sum_{l \in I} \sigma_{i l} \sigma_{l j} \leq \sigma_{i j}
$$

Finally if $t=-r$ then $\sigma_{r,-r}=\sum_{l \neq \pm r} \sigma_{r l} \sigma_{l,-r}+\left\langle\Gamma_{r}\right\rangle$. By (12) it follows that

$$
a_{i r}\left\langle\Gamma_{r}\right\rangle a_{-r, j}^{\prime} \leq \sigma_{i j}
$$

It's only left to notice that

$$
a_{i r} \sigma_{r l} \sigma_{l,-r} a_{r j}^{\prime}=\sum_{k}\left(a_{i r} \sigma_{r l} a_{l k}^{\prime}\right)\left(a_{k l} \sigma_{l,-r} a_{-r, j}^{\prime}\right) \leq \sum_{k} \sigma_{i k} \sigma_{k j} \leq \sigma_{i j}
$$

Therefore, any matrix $a$ in the transporter satisfies condition (T1). In particular, we can apply Proposition 4.2 to any such matrix $a$.

Pick any short $(\sigma, \Gamma)$-elementary transvection $T_{j k}(\xi)$. By Proposition 4.2 we get

$$
S_{i,-i}\left(a T_{j k}(\xi) a^{-1}\right) \equiv a_{i j}^{2} \xi^{2} S_{k,-k}\left(a^{-1}\right)+a_{i,-k}^{2} \xi^{2} S_{-j, j}\left(a^{-1}\right) \quad \bmod \Gamma_{i} .
$$


Now, given a long $(\sigma, \Gamma)$-elementary transvection $T_{j,-j}(\alpha)$ we obtain by the same proposition

$$
S_{i,-i}\left(a T_{j,-j}(\alpha) a^{-1}\right) \equiv a_{i j}^{2} \alpha+a_{i j}^{2} \alpha^{2} S_{-j, j}\left(a^{-1}\right) \quad \bmod \Gamma_{i} .
$$

Given two short $(\sigma, \Gamma)$-elementary transvections $T_{j k}(\xi)$ and $T_{k m}(\zeta)$ such that $j \neq \pm m$ we get

$$
\begin{aligned}
S_{i,-i}\left(a T_{j k}(\xi) T_{k m}(\zeta) a^{-1}\right) \equiv & a_{i j}^{2} \xi^{2} S_{k,-k}\left(a^{-1}\right)+a_{i,-k}^{2} \xi^{2} S_{-j, j}\left(a^{-1}\right) \\
& +a_{i k}^{2} \zeta^{2} S_{m,-m}\left(a^{-1}\right)+a_{i,-m}^{2} \zeta^{2} s_{-k, k}\left(a^{-1}\right) \\
& +a_{i j}^{2} \xi^{2} \zeta^{2} S_{m,-m}\left(a^{-1}\right) \bmod \Gamma_{i} .
\end{aligned}
$$

Finally given a short and a long $(\sigma, \Gamma)$-elementary transvections $T_{j k}(\xi)$ and $T_{k,-k}(\alpha)$ we get

$$
\begin{aligned}
S_{i,-i}\left(a T_{j k}(\xi) T_{k,-k}(\zeta) a^{-1}\right) \equiv & a_{i j}^{2} \xi^{2} S_{k,-k}\left(a^{-1}\right)+a_{i,-k}^{2} \xi^{2} S_{-j, j}\left(a^{-1}\right) \\
& +a_{i k}^{2} \alpha+a_{i k}^{2} \alpha^{2} S_{-k, k}\left(a^{-1}\right) \\
& +a_{i j}^{2} \xi^{2} \alpha^{2} S_{-k, k}\left(a^{-1}\right) \bmod \Gamma_{i} .
\end{aligned}
$$

Comparing (16) and (14) we get the inclusions

$$
a_{i j}^{2} \sigma_{j k}^{2} \sigma_{k m}^{2} S_{k,-k}\left(a^{-1}\right) \in \Gamma_{i}
$$

for all $i, j, k, m \in I$ such that $j \neq \pm k, \pm m$ and $k \neq \pm m$. Similarly comparing (17) with (15) and (14) we get the inclusions

$$
a_{i j}^{2} \sigma_{j m}^{22} \Gamma_{m}^{2} S_{-m, m}\left(a^{-1}\right) \in \Gamma_{i}
$$

for all $i, j, m \in I$ such that $j \neq \pm m$.

Now let $j, m \in I$ such that $j \neq \pm m$. As $h(\nu) \geq(4,3)$ either there exists an index $k \sim m$ such that $k \neq \pm j, \pm m$ or $-m \sim m$. In the first case we get

$$
a_{i j}^{2} \sigma_{j m}^{2} S_{m,-m}\left(a^{-1}\right)=a_{i j}^{2} \sigma_{j k}^{2} R^{2} S_{m,-m}\left(a^{-1}\right)=a_{i j}^{2} \sigma_{j k}^{2} \sigma_{k m}^{2} S_{m,-m}\left(a^{-1}\right) \leq \Gamma_{i}
$$

for all $i \in I$. In the second case we get similarly

$$
a_{i j}^{2} \sigma_{j m}^{[2]} S_{m,-m}\left(a^{-1}\right)=a_{i j}^{2} \sigma_{j,-m}^{[2]} R^{[2]} S_{m,-m}\left(a^{-1}\right)=a_{i j}^{2} \sigma_{j,-m}^{[2]} \Gamma_{-m}^{2]} S_{m,-m}\left(a^{-1}\right) \leq \Gamma_{i}
$$

for all $i \in I$. To prove the inclusions (T2) for the matrix $a$ it's only left to consider the cases when $m=j$ and $m=-j$. Fix an index $k \sim j$ such that $k \neq \pm j$. Observe that

$$
1=\left(\sum_{t \in I} a_{k t}^{\prime} a_{t k}\right)^{2} \equiv \sum_{t \in I} a_{k t}^{\prime 2} a_{t k}^{2} \quad \bmod 2 R
$$

and therefore

$$
\begin{aligned}
a_{i j}^{2} \sigma_{j m}^{2]} S_{m,-m}\left(a^{-1}\right)= & a_{i j}^{2} \sigma_{j k}^{2}\left(\sum_{t \in I} a_{k t}^{\prime} a_{t k}\right)^{2} \sigma_{k m}^{[2} S_{m,-m}\left(a^{-1}\right) \\
& \equiv \sum_{t}\left(a_{i j}^{2} \sigma_{j k}^{[2}\left(a_{k t}^{\prime 2}\right)\right)\left(a_{t k}^{2} \sigma_{k m}^{2} S_{m,-m}\left(a^{-1}\right)\right) \\
& \leq \sum_{t \in I} \sigma_{i t}^{2} \Gamma_{t} \leq \Gamma_{i}
\end{aligned}
$$


where the congruence is meant modulo $\Gamma_{i}$.

Thus $a$ satisfies condition (T2). Finally using (15) and (T2) we get the inclusions (T3) for all $i, j \in I$. Thus we have proved that

$$
\operatorname{Transp}_{\mathrm{Sp}(2 n, R)}(\operatorname{Ep}(\sigma, \Gamma), \operatorname{Sp}(\sigma, \Gamma)) \leq N \text {. }
$$

Finally, it is easy to see that Transp is contravariant in the first variable and therefore

$$
\mathrm{N}_{\mathrm{Sp}(2 n, R)}(\operatorname{Sp}(\sigma, \Gamma)) \leq \operatorname{Transp}_{\mathrm{Sp}(2 n, R)}(\operatorname{Ep}(\sigma, \Gamma), \operatorname{Sp}(\sigma, \Gamma)) \text {. }
$$

Hence also

$$
\mathrm{N}_{\mathrm{Sp}(2 n, R)}(\operatorname{Sp}(\sigma, \Gamma))=\operatorname{Transp}_{\mathrm{Sp}(2 n, R)}(\operatorname{Ep}(\sigma, \Gamma), \operatorname{Sp}(\sigma, \Gamma))=N
$$

\section{Standard setting}

From this section on we focus on proving Theorem 1. Our proof is a loclization based proof. In order to avoid all the hustle with zero divisors, injectivity of the localization morphism and so forth we introduce the concept of a standard setting. In the end of this section we present two conceptual examples of standard settings which motivate the definition.

Let $R$ be a commutative associative unital ring, $R^{\prime}$ a unital subring of $R$ and $S$ a subset of the intersection $R^{\prime} \cap R^{*}$, where $R^{*}$ stands for the set of invertible elements of the ring $R$. We call the triple $\left(R, R^{\prime}, S\right)$ a standard setting if for any $\xi \in R$ there exist an element $x$ in $S$ such that $x \xi \in R^{\prime}$. Clearly, the canonical ring homomorphism $S^{-1} R^{\prime} \rightarrow R$ is an isomorphism. Now let $\left(\sigma^{\prime}, \Gamma^{\prime}\right)$ be an exact form net of ideals of rank $2 n$ over $R^{\prime}$ such that $[\nu]_{R^{\prime}} \leq\left(\sigma^{\prime}, \Gamma^{\prime}\right)$. For each $i, j \in I$ set

$$
\begin{aligned}
\sigma_{i j} & =\left\{\xi \in R \mid \exists x \in S x \xi \in \sigma_{i j}^{\prime}\right\} \\
\Gamma_{i} & =\left\{\alpha \in R \mid \exists x \in S x^{2} \alpha \in \Gamma_{i}^{\prime}\right\} .
\end{aligned}
$$

We will call the pair $(\sigma, \Gamma)$ the $S$-closure of the form net of ideals $\left(\sigma^{\prime}, \Gamma^{\prime}\right)[$ in $R]$. We will show (Proposition 5.1) that $S$-closures of exact form D-nets of ideals over $R^{\prime}$ are exact form D-nets of ideals over $R$.

Fix a subgroup $H$ of $\operatorname{Sp}(2 n, R)$. We call a form net of ideals $\left(\sigma^{\prime}, \Gamma^{\prime}\right)$ over $R^{\prime} S$-associated with the subgroup $H$ if the following two conditions are fulfilled:

1. $\operatorname{Ep}\left(\sigma^{\prime}, \Gamma^{\prime}\right) \leq H$

2. For any elementary symplectic transvection $T_{s r}(\xi)$ contained in $H$ there exists an element $x \in S$ such that $x^{\left(1+\delta_{r,-s}\right)} \xi \in\left(\sigma^{\prime}, \Gamma^{\prime}\right)_{s r}$. 
It is easy to see that a subgroup may have several different $S$-associated nets, but their $S$-closures in $R$ will coincide.

We will introduce now a family of net-like objects. For an arbitrary $g \in \operatorname{Sp}(2 n, R)$ set

$$
\begin{aligned}
\sigma_{i j}^{g} & =\left\{\xi \in R \mid \exists x \in S \forall \theta \in R^{\prime}{ }^{g} T_{i j}(x \theta \xi) \in H\right\}, i \neq \pm j \\
\sigma_{i i}^{g} & =R \\
\Gamma_{i}^{g} & =\left\{\alpha \in R \mid \exists x \in S \forall \theta \in R^{\prime g} T_{i,-i}\left(x^{2} \theta^{2} \alpha\right) \in H\right\} \\
\sigma_{i,-i}^{g} & =\sum_{j \neq \pm i} \sigma_{i j}^{g} \sigma_{j,-i}^{g}+\left\langle\Gamma_{i}^{g}\right\rangle_{R},
\end{aligned}
$$

where the product $\sigma_{i j}^{g} \sigma_{j,-i}^{g}$ denotes the product of ideals, that is the ideal generated by all products $\xi \zeta$, where $\xi \in \sigma_{i j}^{g}$ and $\zeta \in \sigma_{j,-i}^{g}$. In general there is no guarantee that the objects $\left(\sigma^{g}, \Gamma^{g}\right)$, defined in the obvious way from the above data are form nets of ideals. We will show that in cases of interest to us the objects $\left(\sigma^{g}, \Gamma^{g}\right)$ are form nets of ideals and coincide with the $S$-closure of any net which is $S$-associated with the subgroup $H$.

For the rest of this section we fix a standard setting $\left(R, R^{\prime}, S\right)$, a unitary equivalence relation $\nu$ and a subgroup $H$ of $\operatorname{Sp}(2 n, R)$.

Proposition 5.1. Let $\left(\sigma^{\prime}, \Gamma^{\prime}\right)$ be an exact major form net of ideals over $R^{\prime}$ and $(\sigma, \Gamma)$ the $S$-closure of $\left(\sigma^{\prime}, \Gamma^{\prime}\right)$ in $R$. Then $(\sigma, \Gamma)$ is an exact major form net of ideals over $R$. Further, assume that $h(\nu) \geq(4,3)$ and that $\left(\sigma^{\prime}, \Gamma^{\prime}\right)$ is $S$-associated with the subgroup $H$. Then the form net of ideals $(\sigma, \Gamma)$ is coordinate-wise equal to $\left(\sigma^{e}, \Gamma^{e}\right)$.

Proof. Clearly $\sigma_{i j}=R$ whenever $i \sim j$ and $\Gamma_{i}=R$ whenever $i \sim-i$. We will show first that for all $i, j \in I$ the sets $\sigma_{i j}$ and $\Gamma_{i}$ are additive subgroups of $R$. Let $\xi, \zeta \in(\sigma, \Gamma)_{i j}$. By definition, there exist elements $x, y$ in $S$ such that $x^{\left(1+\delta_{j,-i}\right)} \xi, y^{\left(1+\delta_{j,-i}\right)} \zeta \in\left(\sigma^{\prime}, \Gamma^{\prime}\right)_{i j}$. As $\left(\sigma^{\prime}, \Gamma^{\prime}\right)$ is a form net of ideals, it follows that $(x y)^{\left(1+\delta_{j,-i}\right)} \xi,(x y)^{\left(1+\delta_{j,-i}\right)} \zeta \in\left(\sigma^{\prime}, \Gamma^{\prime}\right)_{i j}$ and thus also $(x y)^{\left(1+\delta_{j,-i}\right)}(\xi+\zeta) \in\left(\sigma^{\prime}, \Gamma^{\prime}\right)_{i j}$. Therefore $\xi+\zeta \in(\sigma, \Gamma)_{i j}$. The rest of the properties of $(\sigma, \Gamma)$ as an exact form net of ideals can be deduced in the same way from the corresponding properties of $\left(\sigma^{\prime}, \Gamma^{\prime}\right)$.

Assume $h(\nu) \geq(4,3)$. It's obvious that $\left(\sigma^{e}, \Gamma^{e}\right)_{i j} \leq(\sigma, \Gamma)_{i j}$ for all possible indices $i$ and $j$ and thus also that $\sigma_{i,-i}^{e} \leq \sigma_{i,-i}$ for all $i \in I$. The reverse inclusions are obtained in the following way. Fix some $i \nsim j$ and $\xi \in(\sigma, \Gamma)_{i j}$. By definition, there exists an element $x \in S$ such that $T_{i j}\left(x^{\left(1+\delta_{i,-j}\right)} \xi\right) \in H$. Assume first, $i \neq-j$. Then, as $h(\nu) \geq(4,3)$, there exists another index $k \sim j$ such that $k \neq \pm j, \pm i$. Then $T_{j k}(\theta), T_{k j}(1) \in H$ for all $\theta \in R^{\prime}$ and therefore

$$
T_{i j}(x \theta \xi)=\left[\left[T_{i j}(x \xi), T_{j k}(\theta)\right], T_{k j}(1)\right] \in H .
$$

Hence, $\xi \in \sigma_{i j}^{e}$. If $i=-j$ then there exists another index $k \sim i$ such that $k \neq \pm i$. As $(\sigma, \Gamma)$ is an exact form net of ideals, it follows by Proposition 3.5 that $x^{2} \xi \in \Gamma_{k,-k}^{\prime}$. Thus we get

$$
T_{i,-i}\left(-\varepsilon_{i} \varepsilon_{j} x^{2} \theta^{2} \xi\right) T_{k,-i}\left(x^{2} \theta \xi\right)=\left[T_{k,-k}\left(x^{2} \xi\right), T_{-k,-i}(\theta)\right] \in H .
$$

If $k \sim-i$, then $T_{k,-i}\left(x^{2} \theta \xi\right) \in H$ and therefore $T_{i,-i}\left(-\varepsilon_{i} \varepsilon_{j} x^{2} \theta^{2} \xi\right) \in H$. If $k \nsim-i$, there exists another index $l \sim k$ such that $l \neq \pm k, \pm i$. By relation $(\mathrm{R} 4)$

$$
T_{k,-i}\left(-x^{2} \theta \xi\right)=\left[T_{k l}(1),\left[T_{l k}(-1),\left[T_{k,-k}\left(x^{2} \xi\right), T_{-k,-i}(\theta)\right]\right]\right] \in H .
$$


Therefore $T_{i,-i}\left(-\varepsilon_{i} \varepsilon_{j} x^{2} \theta^{2} \xi\right) \in H$ and $\xi \in \Gamma_{i}^{e}$. Summing up, $(\sigma, \Gamma)_{i j} \leq(\sigma, \Gamma)_{i j}^{e}$ for all $i, j \in I$. As $(\sigma, \Gamma)$ is exact, it follows that $\sigma_{i,-i} \leq \sigma_{i,-i}^{e}$. This completes the proof.

The last proposition allows us to consider the elementary form net subgroup $\operatorname{Ep}(\sigma, \Gamma)$ of $\operatorname{Sp}(2 n, R)$. The following proposition establishes certain properties of the objects $\left(\sigma^{g}, \Gamma^{g}\right)$ which follow directly from their definition and the Steinberg relations. This shows that $\left(\sigma^{g}, \Gamma^{g}\right)$ is "almost a form net of ideals".

Proposition 5.2. Assume $h(\nu) \geq(4,3)$. Let $g$ be an element of $\operatorname{Ep}(\sigma, \Gamma)$. If $[\nu]_{R} \leq$ $\left(\sigma^{g}, \Gamma^{g}\right)$ coordinate-wise then the following inclusions hold:

1. $\sigma_{i j}^{g} \sigma_{j k}^{g} \leq \sigma_{i k}^{g}$ for all $i \neq \pm j, j \neq \pm k$

2. $\Gamma_{i}^{g} \sigma_{-i, k}^{g} \leq \sigma_{i k}^{g}$ and $\sigma_{i,-k}^{g} \Gamma_{-k}^{g} \leq \sigma_{i k}^{g}$ for all $i, k \in I$

3. $\left(\sigma_{i j}^{g}\right)^{\mathscr{2}^{2}} \Gamma_{j}^{g} \leq \Gamma_{i}^{g}$ for all $i \neq \pm j$

4. $2 \sigma_{i j}^{g} \sigma_{j,-i}^{g} \leq \Gamma_{i}^{g}$ for all $i \neq \pm j$,

where products are Minkowski products of sets, i.e. sets of products of elements of the factors.

Proof. 1. The first property follows directly from the Steinberg relation (R4). Indeed, pick any $\xi \in \sigma_{i j}$ and any $\zeta \in \sigma_{j k}$ such that $i \neq \pm j, \pm k$ and $j \neq \pm k$. Then there exist elements $x_{\xi}, x_{\zeta} \in S$ such that ${ }^{g} T_{i j}\left(x_{\xi} \xi\right),{ }^{g} T_{j k}\left(x_{\zeta} \theta \zeta\right) \in H$ for all $\theta \in R^{\prime}$. By relation (R4) we get

$$
{ }^{g} T_{i k}\left(x_{\xi} x_{\zeta} \theta \xi \zeta\right)=\left[{ }^{g} T_{i j}\left(x_{\xi} \xi\right),{ }^{g} T_{j k}\left(x_{\zeta} \theta \zeta\right)\right] \in H
$$

for all $\theta \in R^{\prime}$. Therefore $\xi \zeta \in \sigma_{i k}^{g}$. The corresponding inclusions for the cases when $i= \pm k$ trivially follow from the definition of $\left(\sigma^{g}, \Gamma^{g}\right)$.

2. The second inclusion is trivial when $i= \pm k$ for the same reason as above. Assume $i \neq \pm k$. We will prove the inclusion $\Gamma_{i}^{g} \sigma_{-i, k}^{g} \leq \sigma_{i k}^{g}$. The other one can be treated similarly. Pick any $\alpha \in \Gamma_{i}$ and $\xi \in \sigma_{-i, k}$. Then there exist elements $x_{\alpha}, x_{\xi} \in S$ such that for any $\theta \in R^{\prime}$ we have

$$
{ }^{g} T_{i,-i}\left(x_{\alpha}^{2} \alpha\right),{ }^{g} T_{-i, k}\left(x_{\xi} \theta \xi\right) \in H .
$$

By relation (R6) it follows that

$$
{ }^{g} T_{i k}\left(x_{\alpha}^{2} x_{\xi} \theta \alpha \xi\right){ }^{g} T_{-k, k}\left(x_{\alpha}^{2} x_{\xi}^{2} \theta^{2} \alpha \xi^{2}\right)=\left[{ }^{g} T_{i,-i}\left(x_{\alpha}^{2} \alpha\right),{ }^{g} T_{-i, k}\left(x_{\xi} \theta \xi\right)\right] \in H .
$$

If $k \sim-k$ then by the definition of $\Gamma_{-k}^{g}$ we get ${ }^{g} T_{-k, k}\left(x_{\alpha}^{2} x_{\xi}^{2} \theta^{2} \alpha \xi^{2}\right) \in H$. Thus we get ${ }^{g} T_{i k}\left(x_{\alpha}^{2} x_{\xi} \theta \alpha \xi\right) \in H$ and $\alpha \xi \in \sigma_{i k}^{g}$. If $k \nsim-k$ then, as $h(\nu) \geq(4,3)$, there exists another index $l \sim i$ such that $l \neq \pm i, \pm k$. Then there exist elements $x_{1}, x_{2} \in S$ such that ${ }^{g} T_{l i}\left(x_{1}\right),{ }^{g} T_{i l}\left(x_{2}\right) \in H$. By the Steinberg relations (R3) and (R4) together with (18) we get

$$
{ }^{g} T_{i k}\left(x_{1} x_{2} x_{\alpha}^{2} x_{\xi} \theta \alpha \xi\right)=\left[{ }^{g} T_{i l}\left(x_{2}\right),\left[{ }^{g} T_{l i}\left(x_{1}\right),{ }^{g} T_{i k}\left(x_{\alpha}^{2} x_{\xi} \theta \alpha \xi\right){ }^{g} T_{-k, k}\left(x_{\alpha}^{2} x_{\xi}^{2} \theta^{2} \alpha \xi^{2}\right)\right]\right] \in H
$$

for all $\theta \in R^{\prime}$. It follows that $\alpha \xi \in \sigma_{i k}^{g}$. 
3. The next series of inclusions is established similarly. Fix some indices $i \neq \pm j$, an element $\xi \in \sigma_{i j}^{g}$ and an element $\alpha \in \Gamma_{j}^{g}$. Then there exist elements $x_{\xi}, x_{\alpha} \in S$ such that

$$
{ }^{g} T_{i,-j}\left(x_{\xi} x_{\alpha}^{2} \theta \xi \alpha\right) \cdot T_{i,-i}\left(x_{\xi}^{2} x_{\alpha}^{2} \theta^{2} \xi^{2} \alpha\right)=\left[{ }^{g} T_{i j}\left(x_{\xi} \theta \xi\right),{ }^{g} T_{j,-j}\left(x_{\alpha}^{2} \alpha\right)\right] \in H
$$

for all $\theta \in R^{\prime}$. By assertion (2) of the current lemma, the first term of the left-hand side of (19) is contained in $H$ whenever $\theta$ is a multiple of some $x_{0} \in S$. Therefore the second term of (19) is also contained in $H$ for the same values of parameter $\theta$. This shows that $\xi^{2} \alpha \in \Gamma_{i}^{g}$.

4. Finally, fix an index $i \neq \pm j$, an element $\xi \in \sigma_{i j}^{g}$ and an element $\zeta \in \sigma_{j,-i}^{g}$. Then there exist $x_{\xi}, x_{\zeta} \in S$ such that ${ }^{g} T_{i j}\left(x_{\xi} \theta \xi\right),{ }^{g} T_{j,-i}\left(x_{\zeta} \theta \zeta\right) \in H$ for all $\theta \in R^{\prime}$; in particular ${ }^{g} T_{i j}\left(x_{\xi} x_{\zeta} \theta \xi\right),{ }^{g} T_{j,-i}\left(x_{\zeta} x_{\xi} \theta \zeta\right) \in H$ for all $\theta \in R^{\prime}$. By the Steinberg relation (R5) it follows that

$$
{ }^{g} T_{i,-i}\left(2 x_{\xi}^{2} x_{\zeta}^{2} \theta^{2} \xi \zeta\right) \in H
$$

for all $\theta \in R^{\prime}$. Hence, $\xi \zeta \in \Gamma_{i}$.

Lemma 5.3. Assume $h(\nu) \geq(4,3)$. Let $\left(\sigma^{\prime}, \Gamma^{\prime}\right)$ be an exact major form net of ideals over $R^{\prime}$, which is $S$-associated with $H$. Let $(\sigma, \Gamma)$ denote the $S$-closure of $\left(\sigma^{\prime}, \Gamma^{\prime}\right)$ in $R$. Then for every $g \in \operatorname{Ep}(\sigma, \Gamma)$ the coordinate-wise equality

$$
(\sigma, \Gamma)=\left(\sigma^{g}, \Gamma^{g}\right)
$$

holds. In particular, each such $\left(\sigma^{g}, \Gamma^{g}\right)$ is an exact major form net of ideals over $R$.

Proof. We will prove this lemma by induction on the word length $L(g)$ of $g$ in terms of the generators of $\operatorname{Ep}(\sigma, \Gamma)$. Proposition 5.1 serves as a base of induction, namely it shows that when $L(g)=0$ and $g=e$ we have the equality $(\sigma, \Gamma)=\left(\sigma^{e}, \Gamma^{e}\right)$.

Before proving the induction step, we will prove a slightly stronger statement. Namely, assume $g \in \operatorname{Ep}(\sigma, \Gamma)$ such that $(\sigma, \Gamma) \leq\left(\sigma^{g}, \Gamma^{g}\right)$. Fix an element $T_{p q}(\zeta) \in \operatorname{Ep}(\sigma, \Gamma)$. We will show that $\left(\sigma^{g}, \Gamma^{g}\right) \leq\left(\sigma^{g T_{p q}(\zeta)}, \Gamma^{g T_{p q}(\zeta)}\right)$. Note that, as $(\sigma, \Gamma) \leq\left(\sigma^{g}, \Gamma^{g}\right)$, it follows that $\zeta \in\left(\sigma^{g}, \Gamma^{g}\right)_{p q}$. Fix any $\xi \in\left(\sigma^{g}, \Gamma^{g}\right)_{s r}$ for some indices $s \neq r$. Then there exists an element $x_{\xi} \in S$ such that for every $\theta \in R^{\prime}$ the inclusion ${ }^{g} T_{s r}\left(x_{\xi}^{\kappa} \theta^{\kappa} \xi\right) \in H$ holds, where $\kappa=1+\delta_{s,-r}$. For any $x \in S$ we have the equality

$$
{ }^{g T_{p q}(\zeta)} T_{s r}\left(x^{\kappa} \theta^{\kappa} \xi\right)={ }^{g}\left[T_{p q}(\zeta), T_{s r}\left(x^{\kappa} \theta^{\kappa} \xi\right)\right] \cdot{ }^{g} T_{s r}\left(x^{\kappa} \theta^{\kappa} \xi\right) .
$$

Below we will construct an element $x_{0}$ such that after the substitution $x=x_{0}$ the right-hand side of (21) is contained in $H$ for all $\theta \in R^{\prime}$. It will follow that $\xi \in$ $\left(\sigma^{g T_{p q}(\zeta)}, \Gamma^{g T_{p q}(\zeta)}\right)_{s r}$

Clearly the second term of the right-hand side of (21) is contained in $H$ whenever $x$ is a multiple of $x_{\xi}$. The first term, which we will denote by $h=h(\theta)$, requires a more detailed investigation. First, assume that the transvections $T_{s r}(*)$ and $T_{p q}(*)$ commute. In this case, $h=e$ and thus we can put $x_{0}=x_{\xi}$. Assume that $h \neq e$. The following six alternatives exhaust all possibilities:

(1) $s \neq \pm r, p \neq \pm q$ and one of the following holds 
(i) $s=q, r \neq \pm p$

(ii) $r=-q, s \neq \pm p$

(iii) $s=-p, r \neq \pm q$

(iv) $-r=-p, s \neq \pm q$.

Then $h$ is a single short transvection. We will prove only the case (i). The other ones can be treated similarly. By the Steinberg relation (R4), $h={ }^{g} T_{p r}(x \theta \zeta \xi)$. Recall that

$$
[\nu]_{R} \leq(\sigma, \Gamma) \leq\left(\sigma^{g}, \Gamma^{g}\right) .
$$

By Proposition 5.2 we get $\zeta \xi \in \sigma_{p r}^{g}$. Therefore there exists an element $x_{\zeta} \in S$ such that $h$ is contained in $H$ whenever $x$ is a multiple of $x_{\zeta}$. Put $x_{0}=x_{\xi} x_{\zeta}$.

(2) $p \neq \pm q, s \neq \pm r$ and one of the following holds:

(i) $s=q, r=-p$

(ii) $s=-p, r=q$

(iii) $s=p, r=-q$

(iv) $s=-q, r=p$.

In this case we can compute $h$ using the Steinberg relation (R5). Again, we will prove only the case (i). As $\zeta \in \sigma_{p q}^{g}$, there exists an element $x_{\zeta} \in S$ such that ${ }^{g} T_{p q}\left(x_{\zeta} \zeta\right) \in H$. Then $h \in H$ for all $\theta \in R^{\prime}$ and $x_{0}=x_{\xi} x_{\zeta}$. Indeed,

$$
h=\left[{ }^{g} T_{p q}(\zeta),{ }^{g} T_{q,-p}\left(x_{\zeta} x_{\xi} \theta \xi\right)\right]={ }^{g} T_{p,-p}\left(2 \zeta x_{\zeta} x_{\xi} \theta \xi\right)=\left[{ }^{g} T_{p q}\left(\zeta x_{\zeta}\right),{ }^{g} T_{q,-p}\left(x_{\xi} \theta \xi\right)\right] \in H
$$

for every $\theta \in R^{\prime}$ due to the choice of $x_{\xi}$ and $x_{\zeta}$.

(3) $q=-p, s \neq \pm r$ and either $s=-p$ or $r=p$. In both cases $h$ is a product of a long and a short symplectic elementary transvection. We will consider only the first option. By relation (EU6),

$$
h=\left[{ }^{g} T_{p,-p}(\zeta),{ }^{g} T_{-p, r}(x \theta \xi)\right]={ }^{g} T_{p r}(x \theta \xi \zeta)^{g} T_{-r, r}\left( \pm x^{2} \theta^{2} \xi^{2} \zeta\right) .
$$

By Proposition 5.2 it follows that $\xi \zeta \in \Gamma_{p}^{g} \sigma_{-p, r} \leq \sigma_{p r}^{g}$ and $\xi^{2} \zeta \in\left(\sigma_{-p, r}^{g}\right)^{2} \Gamma_{p}^{g} \leq \Gamma_{-r}^{g}$. Therefore there exist elements $x_{\xi \zeta}, x_{\xi^{2} \zeta} \in S$ such that the first term of the righthand side of (22) belongs to $H$ whenever $x$ is a multiple of $x_{\xi \zeta}$ and the second term whenever $x$ is a multiple of $x_{\xi^{2} \zeta}$. Put $x_{0}=x_{\xi} x_{\xi \zeta} x_{\xi^{2} \zeta}$.

(4) $p \neq \pm q, r=-s$ and either $s=q$ or $s=-p$. Then $h$ is a product a long and a short transvection. We prove only the first option, $s=q$. By the Steinberg relations (R1) and (R6) we have

$$
h=\left[{ }^{g} T_{p q}(\zeta),{ }^{g} T_{q,-q}\left(x^{2} \theta^{2} \xi\right)\right]={ }^{g} T_{p,-q}\left( \pm x^{2} \theta^{2} \xi \zeta\right)^{g} T_{p,-p}\left( \pm x^{2} \theta^{2} \zeta^{2} \xi\right) .
$$

As before, by Proposition 5.2 we have $\xi \zeta \in \sigma_{p q}^{g} \Gamma_{q}^{g} \leq \sigma_{p,-q}^{g}$ and $\zeta^{2} \xi \in\left(\sigma_{p q}^{g}\right)^{2} \Gamma_{q}^{g} \leq \Gamma_{p}^{g}$. Therefore there exist elements $x_{\xi \zeta}, x_{\zeta^{2} \xi} \in S$ such that the right-hand side of (23) is contained in $H$ whenever $x$ is a multiple of $x_{\xi \zeta} x_{\zeta^{2} \xi}$. Put $x_{0}=x_{\xi} x_{\xi \zeta} x_{\zeta^{2} \xi}$. 
(5) Either $s=q, r=p$ or $s=-p, r=-q$. Without loss of generality we can assume the former. In this case, we can't apply any of the Steinberg relations directly, but we can first decompose $T_{s r}(*)$ as a product of transvections for which we know the commutators with $T_{p q}(*)$. As $h(\nu) \geq(4,3)$ there exists either another index $h \sim p$ such that $h \neq \pm p, \pm q$, or $p \sim-p$. In the first case,

$$
\begin{aligned}
{ }^{g T_{p q}(\zeta)} T_{q p}(x \theta \xi) & ={ }^{g T_{p q}(\zeta)}\left[T_{q h}(y \theta \xi), T_{h p}(z)\right] \\
& ={ }^{g}\left[\left[T_{p q}(\zeta), T_{q h}(y \theta \xi)\right] T_{q h}(y \theta \xi),\left[T_{p q}(\zeta), T_{h p}(z)\right] T_{h p}(z)\right] \\
& =\left[{ }^{g} T_{p h}(y \theta \zeta \xi) \cdot{ }^{g} T_{q h}(y \theta \xi),{ }^{g} T_{h q}(-z \zeta) \cdot{ }^{g} T_{h p}(z)\right]
\end{aligned}
$$

whenever $x=y z$. Observe that $\xi \in \sigma_{q p}^{g} \leq \sigma_{q p}^{g} R=\sigma_{q p}^{g} \sigma_{p h}^{g} \leq \sigma_{q h}^{g}, \xi \zeta \in \sigma_{p q}^{g} \sigma_{q h}^{g} \leq \sigma_{p h}^{g}$, $\zeta \in \sigma_{p q}^{g} \leq R \sigma_{p q}^{g}=\sigma_{h p}^{g} \sigma_{p q}^{g} \leq \sigma_{h q}^{g}$ and $1 \in \sigma_{h p}^{g}$. Thus we can choose $y$ and $z$ in $S$ such that all four terms of the right-hand side of (24) are contained in $H$ for all $\theta \in R^{\prime}$. Then we can put $x_{0}=y z x_{\xi}$.

If the equivalence class of $p$ equals $\{ \pm p, \pm q\}$ then we can decompose $T_{s r}(*)$ in a different way, using long transvections. Namely,

$$
\begin{aligned}
{ }^{g T_{p q}(\zeta)} T_{q p}(x \theta \xi)= & { }^{g T_{p q}(\zeta)}\left(\left[T_{q,-q}\left(y^{2}\right), T_{-q, p}(z \theta \xi)\right] T_{-p, p}\left( \pm z^{2} \theta^{2} \xi^{2} y\right)\right)= \\
= & { }^{g}\left(\left[T_{p,-q}\left(\zeta y^{2}\right) T_{p,-p}\left( \pm \zeta^{2} y^{2}\right) T_{q,-q}\left(y^{2}\right),\right.\right. \\
& \left.T_{-q, q}(-2 \zeta z \theta \xi) T_{-q, p}(z \theta \xi)\right] T_{q,-p}\left( \pm \zeta z^{2} \theta^{2} \xi^{2} y^{2}\right) \\
& \left.T_{q,-q}\left( \pm \zeta^{2} z^{2} \theta^{2} \xi^{2} y^{2}\right) T_{-p, p}\left( \pm z^{2} \theta^{2} \xi^{2} y^{2}\right)\right),
\end{aligned}
$$

whenever $x=y^{2} z$. Using the previous cases, we can choose $y$ and $z$ such that the right-hand side of (25) is contained in $H$ for all $\theta \in R^{\prime}$. Put $x_{0}=y^{2} z$.

(6) $q=s=-p, r=p$. Then there exists an index $h \sim p$ such that $h \neq \pm p$. Then

$$
\begin{aligned}
{ }^{g T_{p,-p}(\zeta)} T_{-p, p}\left(x^{2} \theta^{2} \xi\right)= & { }^{g T_{p,-p}(\zeta)}\left(\left[T_{-h, h}\left(y^{2} \theta^{2} \xi\right), T_{h, p}( \pm z)\right] \times\right. \\
& \left.T_{-h, p}\left( \pm y^{2} z \theta^{2} \xi\right)\right) \\
= & { }^{g} T_{-h,-p}\left(y^{2} z \theta^{2} \zeta \xi\right) \cdot{ }^{g} T_{-h, h}\left(y^{4} z^{2} \theta^{2} \xi^{2} \zeta\right) . \\
& { }^{g} T_{-h, p}\left( \pm y^{2} z \theta^{2} \xi\right),
\end{aligned}
$$

whenever $x=y^{2} z$. Observe that

$$
\begin{aligned}
\xi & \in \Gamma_{-p}^{g} \leq R \Gamma_{-p}^{g}=\sigma_{-h,-p}^{g} \Gamma_{-p}^{g} \leq \sigma_{-h, p}^{g} \\
\xi \zeta & \in \sigma_{-h, p}^{g} \Gamma_{p}^{g} \leq \sigma_{-h,-p}^{g} \\
\xi^{2} \zeta & \in\left(\sigma_{-h, p}^{g}\right)^{[2} \Gamma_{p}^{g} \leq \Gamma_{-h}^{g} .
\end{aligned}
$$

Hence we can choose elements $y$ and $z$ in $S$ such that every term of the right-hand side of (26) is contained in $H$ for all $\theta \in R^{\prime}$. Put $x_{0}=y^{2} z$.

The alternatives above are exhaustive. Therefore $\left(\sigma^{g}, \Gamma^{g}\right)_{s r} \leq\left(\sigma^{g T_{p q}(\zeta)}, \Gamma^{g T_{p q}(\zeta)}\right)_{s r}$ for all $s \neq r \in I$. The inclusions $\sigma_{i i}^{g T_{p q}(\zeta)} \leq \sigma_{i i}^{g}$ and $\sigma_{i,-i}^{g T_{p q}(\zeta)} \leq \sigma_{i,-i}^{g}$ follow easily from 
the definition of $\left(\sigma^{g}, \Gamma^{g}\right)$. Therefore we have proved that $\left(\sigma^{g}, \Gamma^{g}\right) \leq\left(\sigma^{g T_{p q}(\zeta)}, \Gamma^{g T_{p q}(\zeta)}\right)$ coordinate-wise, whenever $(\sigma, \Gamma) \leq\left(\sigma^{g}, \Gamma^{g}\right)$.

The induction step looks as follows. Assume that for all elements $g \in \operatorname{Ep}(\sigma, \Gamma)$ such that $L(g) \leq L_{0}$, the equality (20) holds. Let $T_{p q}(\zeta)$ be an elementary transvection in $\operatorname{Ep}(\sigma, \Gamma)$ such that $L\left(g \cdot T_{p q}(\zeta)\right)=L_{0}+1$. Then, as we have proved above, $\left(\sigma^{g}, \Gamma^{g}\right) \leq$ $\left(\sigma^{g T_{p q}(\zeta)}, \Gamma^{g T_{p q}(\zeta)}\right)$, in particular $(\sigma, \Gamma) \leq\left(\sigma^{g T_{p q}(\zeta)}, \Gamma^{g T_{p q}(\zeta)}\right)$. For the same reason

$$
\left(\sigma^{g T_{p q}(\zeta)}, \Gamma^{g T_{p q}(\zeta)}\right) \leq\left(\sigma^{g T_{p q}(\zeta) T_{p q}(-\zeta)}, \Gamma^{g T_{p q}(\zeta) T_{p q}(-\zeta)}\right)=\left(\sigma^{g}, \Gamma^{g}\right)
$$

Summing up, by induction we get the required equality (20) for all $g \in \operatorname{Ep}(\sigma, \Gamma)$.

We will also use the lemma above in the form of the following obvious corollary. It represents the concept of a common denominator for a finite family of fractions.

Corollary 5.4. Assume $h(\nu) \geq(4,3)$. Let $\left(\sigma^{\prime}, \Gamma^{\prime}\right)$ be an exact major form net of ideals which is $S$-associated with the subgroup $H \geq \operatorname{Ep}(\nu, R)$ and let $(\sigma, \Gamma)$ be the $S$-closure of $\left(\sigma^{\prime}, \Gamma^{\prime}\right)$ in $R$. Then for any finite family $\left\{T_{s_{i}, r_{i}}\left(\xi_{i}\right)\right\}_{i \in L}$ of $(\sigma, \Gamma)$-elementary transvections and any finite family $\left\{g_{i}\right\}_{i \in K}$ of elements of $\operatorname{Ep}(\sigma, \Gamma)$ there exists an element $x \in S$ such that

$$
{ }^{g_{i}} T_{s_{j}, r_{j}}\left((x \theta)^{\left(1+\delta_{s_{j},-r_{j}}\right)} \xi_{j}\right) \in H
$$

for all $i \in K, j \in J$ and $\theta \in R^{\prime}$.

\section{Examples of standard settings}

Before we continue with studying the properties of $S$-associated form nets of ideals and their closures, let's consider two main examples of a standard setting. In fact, exactly the opportunity to treat these two cases uniformly was the main motivation to consider this concept at the first place.

1. Trivial standard setting. Let $\widetilde{R}$ be a commutative ring. Let $R=R^{\prime}=\widetilde{R}$ and $S=\{1\}$. Clearly, $\left(R, R^{\prime}, S\right)$ is a standard setting. In this case the concepts of the form net of ideals, associated with a subgroup $H$, a form net of ideals $S$-associated with $H$ and the closure of such coincide.

2. Local standard setting. Let $R$ be a commutative ring and $\mathfrak{m}$ a maximal ideal of $R$. Let $S$ denote the compliment $R \backslash \mathfrak{m}, R_{\mathfrak{m}}$ the localization $S^{-1} R$ and $F_{\mathfrak{m}}$ the localization morphism $R \rightarrow R_{\mathfrak{m}}$. Finally, let $R_{\mathfrak{m}}^{\prime}=F_{\mathfrak{m}}(R)$ and $S_{\mathfrak{m}}=F_{\mathfrak{m}}(S)$. Then $\left(R_{\mathfrak{m}}, R_{\mathfrak{m}}^{\prime}, S_{\mathfrak{m}}\right)$ is a standard setting. Given a subgroup $H$ of $\operatorname{Sp}(2 n, R)$ containing $\operatorname{Ep}(\nu, R)$ with $h(\nu) \geq(4,5)$ and the form net of ideals $(\sigma, \Gamma)$ associated with $H$ we can consider the coordinate-wise image $\left(\sigma_{\mathfrak{m}}^{\prime}, \Gamma_{\mathfrak{m}}^{\prime}\right)$ of $(\sigma, \Gamma)$, i.e.

$$
\sigma_{\mathfrak{m}, i j}^{\prime}=F_{\mathfrak{m}}\left(\sigma_{i j}\right) \text { and } \Gamma_{\mathfrak{m}, i}^{\prime}=F_{\mathfrak{m}}\left(\Gamma_{i}\right) \text { for any } i, j \in I \text {. }
$$

It's easy to see that $\left(\sigma_{\mathfrak{m}}^{\prime}, \Gamma_{\mathfrak{m}}^{\prime}\right)$ is an exact form net of ideals over $R_{\mathfrak{m}}^{\prime}$. We will show in Section 9 that if $R$ is Noetherian then $\left(\sigma_{\mathfrak{m}}^{\prime}, \Gamma_{\mathfrak{m}}^{\prime}\right)$ is $S_{\mathfrak{m}}$-associated with $H$. 


\section{Extraction of transvections}

In this section we perform the extraction of transvections first using matrices in small parabolic subgroups and then using long and short root elements. The methods of this section are rather standard and mostly mimic those of [Vav93], [Vav08], [Vav88] and Vav04. Nontheless, we can't simply refer to the similar results of the cited papers. For our purpuses we have to keep track of denominators while working in a localization of the ground ring. For this sake it is convenient to use the concept of a standard setting.

Throughout this section we fix a standard setting $\left(R, R^{\prime}, S\right)$, a unitary equivalence relation $\nu$, a subgroup $H$ of $\operatorname{Sp}(2 n, R)$ and an exact major form net of ideals $\left(\sigma^{\prime}, \Gamma^{\prime}\right)$ which is $S$-associated with $H$. Let $(\sigma, \Gamma)$ denote the $S$-closure of $\left(\sigma^{\prime}, \Gamma^{\prime}\right)$ in $R$.

\section{Extraction of transvections in parabolic subgroups}

Lemma 6.1. Let a be a matrix in $\operatorname{Sp}(2 n, R)$ such that for some index $p \in I$ the following conditions hold:

1. $a_{p p}=a_{-p,-p}=1$

2. $a_{i j}=\delta_{i j}$ whenever $i \neq-p$ and $j \neq p$.

Then

$$
a=\left(\prod_{1 \leq j \neq \pm p \leq n} T_{-p, j}\left(a_{-p, j}\right) T_{-p,-j}\left(a_{-p,-j}\right)\right) T_{-p, p}\left(S_{-p, p}(a)\right) .
$$

Further, suppose $h(\nu) \geq(4,3)$ and there exists an element $g \in \operatorname{Ep}(\sigma, \Gamma)$ such that ${ }^{g} a \in H$. Then $a \in \operatorname{Ep}(\sigma, \Gamma)$.

Proof. The decomposition (27) can be checked by a straightforward calculation. Suppose $j \neq \pm p$. If $j \sim-p$ then the inclusion $a_{-p, j} \in \sigma_{-p, j}$ is trivial as $\sigma$ is major. From now on, assume $j \nsim-p$. As $h(\nu) \geq(4,3)$, we can choose an index $k \sim j$ such that $k \neq \pm j, \pm p$. Choose using Corollary 5.4 an element $x_{1} \in S$ such that ${ }^{g} T_{j k}\left(x_{1}\right) \in H$. Then

$$
X={ }^{g}\left(T_{-p, k}\left(a_{-p, j} x_{1}\right) T_{-p,-j}\left( \pm a_{-p,-k} x_{1}\right)\right)={ }^{g}\left[a, T_{j k}\left(x_{1}\right)\right] \in H .
$$

If the equivalence class of $j$ is non-self-conjugate then either $j \sim p$ or there exists another index $h \sim j$ such that $h \neq \pm k, \pm j, \pm p$. In the former case, $\pm a_{-p,-k} x_{1} \in \sigma_{-p,-j}^{g}=R$ and by Corollary 5.4 the element $x_{1}$ can be chosen such that $\left.{ }^{g} T_{-p,-j}\left( \pm a_{-p,-k} x_{1}\right)\right) \in H$ and thus also ${ }^{g} T_{-p, k}\left(a_{-p, j} x_{1}\right) \in H$. It follows that $a_{-p, j} \in \sigma_{-p, k}^{g}=\sigma_{-p, j}^{g}$. In the latter case choose using Corollary 5.4 an element $x_{2} \in S$ such that ${ }^{g} T_{k h}\left(x_{2}\right),{ }^{g} T_{h k}\left(x_{2} \theta\right) \in H$ for any $\theta \in R^{\prime}$. Then for the same $\theta$ we get

$$
T_{-p, j}\left(x_{1} x_{2}^{2} \theta a_{-p, j}\right)=\left[\left[X, T_{k h}\left(x_{2}\right)\right], T_{h k}\left(x_{2} \theta\right)\right] \in H .
$$

If the equivalent class of $j$ is self-conjugate, it contains at least the elements $\pm j, \pm k$. Pick using Corollary 5.4 an element $x_{2} \in S$ such that ${ }^{g} T_{k,-j}\left(x_{2}\right),{ }^{g} T_{-j, k}\left(x_{2}\right),{ }^{g} T_{k j}\left(x_{2} \theta\right) \in H$ for all $\theta \in R^{\prime}$. Then

$$
{ }^{g} T_{-p, j}\left(x_{1} x_{2}^{3} \theta a_{-p, j}\right)=\left[\left[\left[X,{ }^{g} T_{k,-j}\left(x_{2}\right)\right],{ }^{g} T_{-j, k}\left(x_{2}\right)\right],{ }^{g} T_{k j}\left(x_{2} \theta\right)\right] \in H .
$$


Therefore $a_{-p, j} \in \sigma_{-p, j}^{g}$ and by Lemma $\lcm{5.3} a_{-p, j} \in \sigma_{-p, j}$ for all $j \neq \pm p$.

In order to prove that $a \in \mathrm{EU}(\sigma, \Gamma)$ it only remains to show that $T_{-p, p}\left(S_{-p, p}(a)\right) \in$ $\operatorname{Ep}(\sigma, \Gamma)$. If $-p \sim p$ then $\Gamma_{-p}=R$ and the inclusion $T_{-p, p}\left(S_{-p, p}(a)\right) \in \operatorname{Ep}(\sigma, \Gamma)$ is trivial. Assume $p \nsim-p$. Set

$$
g_{1}=g \prod_{j>0, j \neq \pm p} T_{-p, j}\left(a_{-p, j}\right) T_{-p,-j}\left(a_{-p,-j}\right)
$$

Then $g_{1} T_{-p, p}\left(S_{-p, p}(a)\right) g^{-1}={ }^{g} a \in H$ and $g_{1}, g^{-1} \in \mathrm{EU}(\sigma, \Gamma)$. As $p \nsim-p$, we can choose two more indices $q$ and $t$ such that $(p, q, t)$ is an $A$-type base triple. Pick an element $y_{1} \in S$ such that ${ }^{g} T_{p q}(y \theta),{ }^{g} T_{p q}(y \theta) \in H$ for all $\theta \in R^{\prime}$ whenever $y$ is a multiple of $y_{1}$. By the Steinberg relation (R6) we have

$$
\begin{array}{r}
{ }^{g_{1}} T_{-p, q}\left(y \theta S_{-p, p}(a)\right) \cdot{ }^{g_{1}} T_{-q, q}\left(-\varepsilon_{p} \varepsilon_{q} y^{2} \theta^{2} S_{-p, p}(a)\right) \cdot{ }^{g_{1}} T_{p q}(y \theta) \\
={ }^{g_{1}}\left[T_{-p, p}\left(S_{-p, p}(a)\right), T_{p q}(y \theta)\right] \cdot{ }^{g_{1}} T_{p q}(y \theta) \\
=\left(g_{1} T_{-p, p}\left(S_{-p, p}(a)\right) g^{-1}\right)\left(g T_{p q}(y \theta) g^{-1}\right)\left(g T_{-p, p}\left(-S_{-p, p}(a)\right) g_{1}^{-1}\right) .
\end{array}
$$

The right-hand side of (28) as well as the third term of the left-hand side of (28) is contained in $H$ whenever $y$ is a multiple of $y_{1}$ in $S$. Therefore

$$
{ }^{g_{1}} T_{-p, q}\left(y \theta S_{-p, p}(a)\right) \cdot{ }^{g_{1}} T_{-q, q}\left(-\varepsilon_{p} \varepsilon_{q} y^{2} \theta^{2} S_{-p, p}(a)\right) \in H
$$

for all $\theta \in S$ whenever $y$ is a multiple of $y_{1}$. Pick $y_{2} \in S$ such that ${ }^{{ }^{1}} T_{-p,-t}\left(y_{2}\right)$, ${ }^{g_{1}} T_{-t,-p}\left(y_{2}\right) \in H$. We get

$$
\begin{array}{r}
{ }^{g_{1}} T_{-p, q}\left(y y_{2}^{2} \theta S_{-p, p}(a)\right)=\left[{ }^{g_{1}} T_{-p,-t}\left(y_{2}\right),\left[{ }^{g_{1}} T_{-t,-p}\left(y_{2}\right),{ }^{g_{1}} T_{-p, q}\left(y \theta S_{-p, p}(a)\right) \cdot\right.\right. \\
\left.\left.{ }^{g_{1}} T_{-q, q}\left(-\varepsilon_{p} \varepsilon_{q} y^{2} \theta^{2} S_{-p, p}(a)\right)\right]\right]
\end{array}
$$

and thus by the choice of $y_{2}$ together with (29) we get that ${ }^{g_{1}} T_{-p, q}\left(y \theta S_{-p, p}(a)\right) \in H$ for all $\theta \in R^{\prime}$ whenever $y$ is a multiple of $y_{1} y_{2}^{2}$. Combining this result again with (29) we get that ${ }^{g_{1}} T_{-q, q}\left(-\varepsilon_{p} \varepsilon_{q} y^{2} \theta^{2} S_{-p, p}(a)\right) \in H$ for all $\theta \in S$ whenever $y$ is a multiple of $y_{1} y_{2}^{2}$. Thus, $S_{-p, p}(a) \in \Gamma_{-q}$ and by Proposition $3.5 S_{-p, p}(a) \in \Gamma_{-p}$. This completes the proof.

Lemma 6.2. Assume $h(\nu) \geq(4,3)$. Let $(p, q)$ be an A-type base pair and let a be an element of $\operatorname{Sp}(2 n, R)$ such that $a_{* p}=e_{* p}$ or $a_{-p, *}=e_{-p, *}$. Assume that there exist elements $g_{1}, g_{2} \in \operatorname{Ep}(\sigma, \Gamma)$ such that $g_{1} a g_{2} \in H$. Then the inclusion $a_{q j} \in \sigma_{q j}$ holds for each $j \neq-p$. If additionally $a \in \operatorname{Sp}(\sigma)$ then also $S_{q,-q}(a) \in \Gamma_{q}$.

Proof. As $a$ is symplectic it's easy to see that the conditions of the lemma provide the equalities $a_{* p}=a_{* p}^{\prime}=e_{* p}$ and $a_{-p, *}=a_{-p, *}^{\prime}=e_{-p, *}$. Choose via Corollary 5.4 an element $x \in S$ such that ${ }_{2}^{-1} T_{p q}(x) \in H$ and consider the matrix

$$
\begin{aligned}
b=a^{-1} T_{p q}(x) a & =e+a_{* p}^{\prime} x a_{q *}-\varepsilon_{p} \varepsilon_{q} a_{*,-q}^{\prime} x a_{-p, *} \\
& =e+e_{* p} x a_{q *}-\varepsilon_{p} \varepsilon_{q} a_{*,-q}^{\prime} x e_{-p, *} .
\end{aligned}
$$


It's easy to see that $b_{i j}=\delta_{i j}$ whenever $i \neq p$ and $j \neq-p$, that $b_{p p}=b_{-p,-p}=1$ and that

$$
{ }^{g_{1}} b=\left(g_{1} a g_{2}\right)\left(g_{2}^{-1} T_{p q}(x) g_{2}\right)\left(g_{2}^{-1} a^{-1} g_{1}^{-1}\right) \in H .
$$

Therefore by Lemma 6.1 the inclusion $b_{p j} \in \sigma_{p j}$ holds for each $j \neq \pm p$. Note that $b_{p j}=x a_{q j}$ whenever $j \neq \pm p$. Therefore $a_{q j} \in \sigma_{q j}$ for all $j \neq-p$. By Lemma 6.2 we also get the inclusion $S_{p,-p}(b) \in \Gamma_{q}$. Assume $a \in \operatorname{Sp}(\sigma)$. By the corollary 3.4 we have

$$
S_{p,-p}(b) \equiv a_{p p}^{\prime 2} x^{2} S_{q,-q}(a)+a_{p,-q}^{\prime 2} x^{2} S_{-p, p}(a) \bmod \Gamma_{p}^{\min } .
$$

Recall that $a_{-p, *}=e_{-p, *}$. Thus $S_{-p, p}(a)=0$. Further $a_{p p}^{\prime}=1$, and therefore $S_{p,-p}(b) \equiv$ $S_{q,-q}(a) \bmod \Gamma_{p}$. Hence, $S_{q,-q}(a) \in \Gamma_{q}$.

Lemma 6.3. Assume $h(\nu) \geq(4,4)$. Let $(p, q)$ be an A-type base pair and a be an element of $\operatorname{Sp}(2 n, R)$ such that $a_{i j}=\delta_{i j}$ whenever $i \neq-p,-q$ and $j \neq p, q$. Assume that there exists an element $g \in \operatorname{Ep}(\sigma, \Gamma)$ such that ${ }^{g} a \in H$. Then the inclusion $a_{k p} \in \sigma_{k p}$ holds for each $k \neq-p,-q$. If additionally $a \in \operatorname{Sp}(\sigma)$ then also $S_{-p, p}(a) \in \Gamma_{-p}$.

Proof. Fix any $k \nsim p$. As $h(\nu) \geq(4,4)$, there exists an index $h \sim k$ such that $h \neq$ $\pm k, \pm p, \pm q$. Pick using Corollary 5.4 an element $x \in S$ such that ${ }^{g} T_{h k}(x) \in H$ and consider the matrix

$$
b=a^{-1} T_{h k}(x) a=e+a_{* h}^{\prime} x a_{k *}-\varepsilon_{h} \varepsilon_{k} a_{*,-k}^{\prime} x a_{-h, *} .
$$

It is easy to see that $b_{h p}=x a_{k p}$ and ${ }^{g} b \in H$. Further, there exists an index $l \sim k$ such that $l \neq \pm h,-p$ and $b_{* l}=e_{* l}$. Indeed, if $k \sim-k$, one can simply take $l=-k$. If the class of $k$ is non-self-conjugate then such $l$ exists due to the condition $h(\nu) \geq(4,4)(l$ can be equal to $-q$ if $-q \sim k$ ). Therefore, by Lemma 6.2 we get $x a_{k p} \in \sigma_{h p}=\sigma_{k p}$. Thus $a_{k p} \in \sigma_{k p}$.

Assume $a \in \operatorname{Sp}(\sigma)$. If the equivalence class of $p$ is self-conjugate, clearly $S_{-p, p}(a) \in$ $R=\Gamma_{-p}$. If the equivalence class of $p$ is non-self-conjugate then, as $h(\nu) \geq(4,4)$, there exists an index $t \sim p$ such that $(p, q, t)$ is an A-type base triple. Consider the matrix

$$
c=T_{-p,-t}\left(-a_{-p,-t}\right) T_{-q,-t}\left(a_{-q,-t}\right) a .
$$

As $t \sim p \sim q$ it follows that $c \in \operatorname{Sp}(\sigma)$. Note that $a_{-t,-t}=1$, hence $c_{*,-t}=e_{*,-t}$ and

$$
g T_{-q,-t}\left(a_{-q,-t}\right) T_{-p,-t}\left(a_{-p,-t}\right) c g^{-1} \in H .
$$

By Lemma 6.2 it follows that $S_{-p, p}(c) \in \Gamma_{-p}$. Finally, by Corollary 3.3 we have

$$
S_{-p, p}(c) \equiv S_{-p, p}(a)+a_{-p,-t}^{2} S_{-t, t}(a) \bmod \Gamma_{-p}
$$

and as $S_{-t, t}(a)=0$ we get $S_{-p, p}(a) \in \Gamma_{-p}$.

Lemma 6.4. Assume $h(\nu) \geq(4,4)$. Let $p$ be an index in I with self-conjugate equivalence class and let $a$ be an element of $\operatorname{Sp}(2 n, R)$ such that $a_{i j}=\delta_{i j}$ whenever $i \neq \pm p$ and $j \neq \pm p$. If there exists an element $g \in \operatorname{Ep}(\sigma, \Gamma)$ such that ${ }^{g} a \in H$, then $a_{k p} \in \sigma_{k p}$ for all $k \in I$. 
Proof. If $k \sim p$ the inclusion $a_{k p} \in \sigma_{k p}$ is trivial. Assume $k \nsim p$, in particular $k \neq \pm p$. As $h(\nu) \geq(4,4)$, there exists another index $h \sim k$ such that $h \neq \pm k \pm p$. Pick using Corollary 5.4 an element $x \in S$ such that ${ }^{g} T_{h k}(x) \in H$ and consider the matrix

$$
b=a^{-1} T_{h k}(x) a=e+a_{* h}^{\prime} m a_{k *}-\varepsilon_{h} \varepsilon_{k} a_{*,-k}^{\prime} m a_{-h, *} .
$$

We will show that it satisfies the conditions of Lemma 6.2. Indeed, by choice of $x$ the inclusion ${ }^{g} b \in H$ holds. Pick an index $q$ such that $(p, q)$ is a C-type base pair. It is easy to see that $q \nsim \pm k$. Clearly $b_{* q}=e_{* q}$. Applying Lemma 6.2 to the matrix $a$, the elementary transvection $T_{h k}(x)$ and the pair $(-p, q)$, we get $b_{-p, j} \in \sigma_{-p, j}$ for all $j \neq-q$. Thus $b_{-p,-h} \in \sigma_{-p,-h}=\sigma_{k p}$ and it is only left to notice that $b_{-p,-h}=$ $-\varepsilon_{h} \varepsilon_{k} a_{-p,-k}^{\prime} x a_{-h,-h}= \pm x a_{k p}$. Therefore $a_{k p} \in \sigma_{k p}$ for all $k \in I$.

\section{Extraction of transvections using root elements}

Lemma 6.5. Assume $h(\nu) \geq(4,4)$. Let $(p, q, h)$ be an A-type base triple, a an element of $\operatorname{Sp}(2 n, R)$ and $T_{s r}(\xi)$ a short elementary transvection. Let $b$ denote the short root element $a T_{s r}(\xi) a^{-1}$. Suppose that $a_{p,-r}=a_{q,-r}=0$. Assume that there exist elements $g_{1}, g_{2} \in \operatorname{Ep}(\sigma, \Gamma)$ such that $g_{1} a g_{2} \in H$ and ${ }^{g_{2}^{-1}} T_{s r}(\xi) \in H$. Then $a_{p s} b_{i h} \in \sigma_{i h}$ for all $i \neq-p,-q$. If additionally $a \in \operatorname{Sp}(\sigma)$ then also $a_{p s}^{2} S_{-h, h}\left(b^{-1}\right) \in \Gamma_{-h}$.

Proof. It is easy to see that ${ }^{g_{1}} b \in H$. Indeed

$$
{ }^{g_{1}} b=\left(g_{1} a g_{2}\right)\left(g_{2}^{-1} T_{s r}(\xi) g_{2}\right)\left(g_{2}^{-1} a^{-1} g_{1}^{-1}\right) \in H .
$$

Using Corollary 5.4 pick an element $x \in S$ such that ${ }^{g_{1}} T_{h p}\left(-a_{q s} x\right),{ }^{g_{1}} T_{h q}\left(a_{p s} x\right) \in H$. Set $\alpha=-a_{q s} x$ and $\beta=a_{p s} x$. Clearly, $\alpha a_{p s}+\beta a_{q s}=0$. Consider the matrix

$$
c=b T_{h p}(\alpha) T_{h q}(\beta) b^{-1}=e+b_{* h}\left(\alpha b_{p *}^{\prime}+\beta b_{q *}^{\prime}\right)-\varepsilon_{h}\left(\varepsilon_{p} \alpha b_{*,-p}+\varepsilon_{q} \beta b_{*,-q}\right) b_{-h, *}^{\prime} .
$$

Obviously, ${ }^{g_{1}} c \in H$ and by the conditions that $a_{p,-r}=a_{q,-r}=0$ and $\alpha a_{p s}+\beta a_{q s}=0$ it easily follows that $\left(\alpha b_{p *}^{\prime}+\beta b_{q *}^{\prime}\right)=\left(\alpha e_{p *}+\beta e_{q *}\right)$ and $\left(\varepsilon_{p} \alpha b_{*,-p}+\varepsilon_{q} \beta b_{*,-q}\right)=\left(\varepsilon_{p} \alpha e_{*,-p}+\right.$ $\left.\varepsilon_{q} \beta e_{*,-q}\right)$. Thus we can rewrite (30) as follows

$$
c=e+b_{* h}\left(\alpha e_{p *}+\beta e_{q *}\right)-\varepsilon_{h}\left(\varepsilon_{p} \alpha e_{*,-p}+\varepsilon_{q} \beta e_{*,-q}\right) b_{-h, *}^{\prime} .
$$

In particular, $c_{i j}=\delta_{i j}$ whenever $i \neq-p,-q$ and $j \neq p, q$. Applying Lemma 6.3 to the matrix $c$ we get the inclusion $c_{i q} \in \sigma_{i q}$ for all $i \neq-p,-q$. Notice that $c_{i q}=\beta b_{i h}=x a_{p s} b_{i h}$ for $i \neq-p,-q$. This completes the proof of the first part of the lemma.

Assume $a \in \operatorname{Sp}(\sigma)$. It follows that $b, c \in \operatorname{Sp}(\sigma)$. Therefore by Lemma 6.3 we get the inclusion $S_{-q, q}(c) \in \Gamma_{-q}$. By Corollary 3.4 we have

$$
\begin{aligned}
S_{-q, q}(c) \equiv & b_{-q, h}^{2} x^{2}\left(a_{q s}^{2} S_{p,-p}\left(b^{-1}\right)+a_{p s}^{2} S_{q,-q}\left(b^{-1}\right)\right) \\
& +\left(b_{-q,-p}^{2} a_{q s}^{2}+b_{-q,-q}^{2} a_{p s}^{2}\right) x^{2} S_{-h, h}\left(b^{-1}\right) \in \Gamma_{-q} .
\end{aligned}
$$


As $S_{-h, h}\left(b^{-1}\right) \in \sigma_{-q, q}$ and $2 \sigma_{-q, q} \leq \Gamma_{i}$ it follows that

$$
\begin{array}{r}
\left(b_{-q,-p}^{2} a_{q s}^{2}+b_{-q,-q}^{2} a_{p s}^{2}\right) x^{2} S_{-h, h}\left(b^{-1}\right) \equiv \\
\left(b_{-q,-p} a_{q s}+b_{-q,-q} a_{p s}\right)^{2} x^{2} S_{-h, h}\left(b^{-1}\right) \quad \bmod \Gamma_{-q} .
\end{array}
$$

A straightforward calculation shows that $b_{-q,-p} a_{q s}+b_{-q,-q} a_{p s} \in a_{p s}+2 R$, which together with (32) yields

$$
\left(b_{-q,-p}^{2} a_{q s}^{2}+b_{-q,-q}^{2} a_{p s}^{2}\right) x^{2} S_{-h, h}\left(b^{-1}\right) \equiv a_{p s}^{2} x^{2} S_{-h, h}\left(b^{-1}\right) \quad \bmod \Gamma_{-q} .
$$

As $a_{p,-r}=a_{q,-r}=0$, we have

$$
\begin{aligned}
& b_{p j}^{\prime}=\delta_{p j}-a_{p s} \xi a_{r j}^{\prime} \\
& b_{q j}^{\prime}=\delta_{q j}-a_{q s} \xi a_{r j}^{\prime} .
\end{aligned}
$$

In particular, $b_{p,-p}^{\prime}=b_{q,-q}^{\prime}=b_{p,-q}^{\prime}=b_{q,-p}^{\prime}=0$. Therefore

$$
\begin{aligned}
a_{q s}^{2} S_{p,-p}\left(b^{-1}\right) & =-\varepsilon_{p} \sum_{j>0, j \neq \pm p, \pm q} a_{q s}^{2} b_{p j}^{\prime} b_{p,-j}^{\prime}=-\varepsilon_{p} \sum_{j>0, j \neq \pm p, \pm q} a_{q s}^{2} a_{p s}^{2} \xi^{2} a_{r j}^{\prime} a_{r,-j}^{\prime} \\
& =\varepsilon_{p} \varepsilon_{q} a_{p s}^{2} S_{q,-q}\left(b^{-1}\right)
\end{aligned}
$$

and thus

$$
b_{-q, h}^{2} x^{2}\left(a_{q s}^{2} S_{p,-p}\left(b^{-1}\right)+a_{p s}^{2} S_{q,-q}\left(b^{-1}\right)\right) \in 2 \sigma_{-q, q} \leq \Gamma_{-q} .
$$

Combining (31), (32), (33) and (34) we get the inclusion $a_{p s}^{2} x^{2} S_{-h, h}\left(b^{-1}\right) \in \Gamma_{-h}$. By the property $(\Gamma 2)$ of a form net of ideals it follows that $a_{p s}^{2} S_{-h, h}\left(b^{-1}\right) \in \Gamma_{-h}$.

Lemma 6.6. Assume $h(\nu) \geq(4,4)$. Let $(p, q)$ be a C-type base pair, a an element of $\operatorname{Sp}(2 n, R)$ and $T_{s,-s}(\xi)$ a long elementary transvection. Let $b$ denote the long root element $a T_{s,-s}(\xi) a^{-1}$. If there exist elements $g_{1}, g_{2} \in \operatorname{Ep}(\sigma, \Gamma)$ such that $g_{1} a g_{2} \in H$ and $g_{2}^{-1} T_{s,-s}(\xi) \in H$, then $a_{p s} b_{i h} \in \sigma_{i h}$ for all $i \in I$.

Proof. Clearly ${ }^{g_{1}} b \in H$. Indeed

$$
{ }^{g_{1}} b=g_{1} a T_{s,-s}(\xi) a^{-1} g_{1}^{-1}=\left(g_{1} a g_{2}\right)\left({ }^{g_{2}^{-1}} T_{s,-s}(\xi)\right)\left(g_{2}^{-1} a^{-1} g_{1}^{-1}\right)
$$

and each term in brackets is contained in $H$. Pick $x \in S$ such that ${ }^{g_{1}} T_{h p}\left(-a_{-p, s} x\right)$, ${ }^{g_{1}} T_{h,-p}\left(a_{p s} x\right) \in H$. Set $\alpha=-a_{-p, s} x$ and $\beta=a_{p s} x$. Clearly $a_{p s} \alpha+a_{-p, s} \beta=0$. It easily follows that $b_{p *}^{\prime} \alpha+b_{-p, *}^{\prime} \beta=e_{p *} \alpha+e_{-p, *} \beta$ and $\left(\varepsilon_{p} \alpha b_{*,-p}+\varepsilon_{-p} \beta b_{* p}\right)=\left(\varepsilon_{p} \alpha e_{*,-p}+\varepsilon_{-p} \beta e_{* p}\right)$. Consider the matrix

$$
\begin{aligned}
c=b T_{h p}(\alpha) T_{h,-p}(\beta) b^{-1} & =e+b_{* h}\left(b_{p *}^{\prime} \alpha+b_{-p, *} \beta\right)-\varepsilon_{h}\left(\varepsilon_{p} \alpha b_{*,-p}+\varepsilon_{-p} \beta b_{* p}\right) b_{-h, *}^{\prime} \\
& =e+b_{* h}\left(e_{p *} \alpha+e_{-p, *} \beta\right)-\varepsilon_{h}\left(\varepsilon_{p} \alpha e_{*,-p}+\varepsilon_{-p} \beta e_{* p}\right) b_{-h, *}^{\prime} .
\end{aligned}
$$

It is easy to see that $c_{i j}=\delta_{i j}$ whenever $i \neq \pm p$ and $j \neq \pm p$. By Lemma 6.4 it follows that $c_{i,-p} \in \sigma_{i p}$ for all $i \in I$. It's only left to notice that $c_{i,-p}=\beta b_{i h}=x a_{p s} b_{i h}$ whenever $i \neq \pm p$. Hence $a_{p s} b_{i h} \in \sigma_{i h}$ for all $i \in I$. 


\section{At the level of the Jacobson radical}

The whole benefit of using localization based proofs is that we can work over a local ring, where every element is either invertible or is contained in the unique maximal ideal, which coincides with the Jacobson radical. Thus, the extraction of transvections over local rings rely on certain elements either being invertible or being contained in the Jacobson radical. In this section, we investigate the latter case. Consider a standard setting $\left(R, R^{\prime}, S\right)$. Let $J$ denote the Jacobson radical of $R$. We will show (Corollary 7.6) that the matirces in an overgroup $H$ of $\operatorname{Ep}\left(\nu, R^{\prime}\right)$ with sufficiently large $h(\nu)$, that are contained in $\operatorname{Sp}(2 n, R, J)$, are in fact contained in $\operatorname{Sp}(\sigma, \Gamma)$, where, as usual, $(\sigma, \Gamma)$ stands for the closure of a net $S$-associated with $H$. In fact we will show that $H \cap \operatorname{Sp}_{J} \leq \operatorname{Sp}(\sigma, \Gamma)$, where $\mathrm{Sp}_{J}$ is some set of matrices larger than $\operatorname{Sp}(2 n, R, J)$.

Throughout this section we fix a standard setting $\left(R, R^{\prime}, S\right)$, a unitary equivalence relation $\nu$, a subgroup $H$ of $\operatorname{Sp}(2 n, R)$ which contains $\operatorname{Ep}\left(\nu, R^{\prime}\right)$ and an exact major form net of ideals $\left(\sigma^{\prime}, \Gamma^{\prime}\right)$ over $R^{\prime}$ which is $S$-associated with $H$. Let $J$ denote the Jacobson radical of the ring $R$ and let $(\sigma, \Gamma)$ denote the $S$-closure in $R$ of the form net of ideals $\left(\sigma^{\prime}, \Gamma^{\prime}\right)$. By definition, every element $x$ of the Jacobson radical $J$ is quasi-regular, i.e. $1+x y \in R^{*}$ for all $y \in R$, in particular $1+x \in R^{*}$. It follows that $R^{*}+J \subseteq R^{*}$. Indeed, let $x$ be invertible and $y \in J$ then, as $y$ is quasi-regular, $1+x^{-1} y \in R^{*}$. Therefore, and $x+y=x\left(1+x^{-1} y\right)$ is a unit since it is a product of two units. We will use this property without reference.

The main idea of this section is as follows. Pick a matrix $a \in H \cap \operatorname{Sp}(2 n, R, J)$. Fix an $A$-type base quintuple $(p, q, h, t, l)$. We would like to show that $a_{i p} \in \sigma_{i p}$ and $S_{-p, p}\left(a^{-1}\right) \in \Gamma_{-p}$. So far the only way of extracting transvections we know is either from an element close to a parabolic subgroup or from a root element. There is no much hope that an arbitrary element of $H \cap \operatorname{Sp}(2 n, R, J)$ is any close to a parabolic subgroup. Thus we have to get by with the second option. Let's construct a root element $b=a T_{s r}(\xi) a^{-1}$ which will preserve the information about the original element $a$. Once we show that $b_{a,-r}=b_{a,-r}=0$ it follows by Lemma 6.5 that

$$
a_{p s}\left(\delta_{i h}+a_{i s} \xi a_{r h}^{\prime}-\varepsilon_{s} \varepsilon_{r} a_{i,-r} \xi a_{-s, h}^{\prime}\right)=a_{p s} b_{i h} \in \sigma_{i h} .
$$

In order to get the inclusion $a_{i p} \in \sigma_{i p}$ we have to put either $s=p$ or $-r=p$. Let's choose $s=p$. Note that in this case $a_{p s}=a_{p p}$ is invertible. In order to get one step closer it would be beneficial to have $a_{r h}^{\prime}$ also invertible. This happens for example when $r=h$. Now suppose that $a_{-p, h}=a_{-s, h}=0$. In this case the inclusion (35) yields the inclusion $a_{i p} \in \sigma_{i p}$ immediately! This is exactly what we do in Lemma 7.3. Lemmas 7.1 and 7.2 serve the goal of getting the condition $a_{-p, h}=0$ fulfilled. The inclusion $S_{-p, p}\left(a^{-1}\right) \in \Gamma_{-p}$ is handled in a similar manner. The case when we work with a C-type pair rather than an A-type quintuple is quite a bit easier and is handled by Lemma 7.5. Everywhere in this section we try to use as little of the assumption that $a \in \operatorname{Sp}(2 n, R, J)$ as possible, namely we explicitly list all the individual inclusions for entries of $a$ that we require. This allows us in future to work not only with a matrix in $H \cap \operatorname{Sp}(2 n, R, J)$ but with any matrix $a \in H$ having a submatrix that looks like a submatrix of a matrix in $\operatorname{Sp}(2 n, R, J)$. 
Lemma 7.1. Assume $h(\nu) \geq(4,4)$. Let $(p, q, h, t, l)$ be an A-type base quintuple and a an element of $\operatorname{Sp}(2 n, R)$ such that $a_{-p,-t}=a_{-h,-t}=a_{-l,-t}=a_{p q}=a_{h q}=0, a_{-p, q} a_{t,-p}^{\prime}$, $a_{-h, q} a_{t,-h}^{\prime} \in J$ and $a_{q q} \in R^{*}$. If there exist elements $g_{1}$ and $g_{2}$ in $\operatorname{Ep}(\sigma, \Gamma)$ such that $g_{1} a g_{2} \in H$ then $a_{p,-t} \in \sigma_{p,-t}$.

Proof. Pick using Corollary 5.4 an element $x \in S$ such that ${ }_{2}^{-1} T_{q t}(x) \in H$ and consider the matrix $b=a T_{q t}(x) a^{-1}$. By choice of the parameter $x$ we have

$$
{ }^{g_{1}} b=\left(g_{1} a g_{2}\right)\left(g_{2}^{-1} T_{q t}(x) g_{2}\right)\left(g_{2}^{-1} a^{-1} a_{1}^{-1}\right) \in H .
$$

Pick using Corollary 5.4 another element $y \in S$ such that ${ }^{g_{1}} T_{-p,-h}(y) \in H$ and consider the matrix $c=b T_{-p,-h}(y) b^{-1}$. Clearly, ${ }^{{ }_{1}} c \in H$ for the same reason as above. We are going to apply Lemma 6.5 to the matrix $b$, the short elementary transvection $T_{-p,-h}(y)$ and the $A$-type base triple $(-p,-l,-h)$. In order to do this we have to show first that $b_{-p, h}=b_{-l, h}=0$. Indeed, by the assumptions of this lemma $a_{t h}^{\prime}=\varepsilon_{t} \varepsilon_{h} a_{-h,-t}=0$ and $a_{-p,-t}=a_{-l,-t}=0$. Thus

$$
\begin{aligned}
b_{-p, h} & =a_{-p, q} x a_{t h}^{\prime}-\varepsilon_{q} \varepsilon_{t} a_{-p,-t} x a_{-q, h}^{\prime}=0 \\
b_{-l, h} & =a_{-l, q} x a_{t h}^{\prime}-\varepsilon_{q} \varepsilon_{t} a_{-l,-t} x a_{-q, h}^{\prime}=0 .
\end{aligned}
$$

As $a_{-p, q} a_{t,-p}^{\prime} \in J$, it follows that

$$
\begin{aligned}
b_{-p,-p} & =1+a_{-p, q} x a_{t,-p}^{\prime}-\varepsilon_{q} \varepsilon_{t} a_{-p,-t} x a_{-q,-p}^{\prime} \\
& =1+a_{-p, q} x a_{t,-p}^{\prime} \in 1+J \subseteq R^{*} .
\end{aligned}
$$

Therefore by Lemma 6.5 we get the inclusions $b_{-p,-p} c_{i,-h} \in \sigma_{i,-h}$ and, as $b_{-p,-p}$ is invertible, also $c_{i,-} \in \sigma_{i,-h}$ for all $i \neq p, l$. In particular,

$$
b_{q,-p} y b_{-h,-h}^{\prime}-\varepsilon_{p} \varepsilon_{h} b_{q h} y b_{p,-h}^{\prime}=c_{q,-h} \in \sigma_{q,-h} .
$$

Recall that $a_{-h, q} a_{t,-h}^{\prime} \in J$ Therefore

$$
b_{-h,-h}^{\prime}=1-a_{-h, q} x a_{t,-h}^{\prime}+\varepsilon_{q} \varepsilon_{t} a_{-h,-t} x a_{-q,-h}^{\prime}=1-a_{-h, q} x a_{t,-h}^{\prime} \in 1+J \subseteq R^{*} .
$$

Observe that

$$
b_{p,-h}^{\prime}=-a_{p q} x a_{t,-h}^{\prime}+\varepsilon_{q} \varepsilon_{t} a_{p,-t} x a_{-q,-h}^{\prime}=0 .
$$

Substituting (37) and (38) into (36) we get the inclusion $b_{q,-p} \in \sigma_{q,-h}=\sigma_{q,-p}$. Finally, recall that $a_{q q} \in R^{*}$ and $a_{-q,-p}^{\prime}=\varepsilon_{q} \varepsilon_{p} a_{p q}=0$. Therefore

$$
a_{q q} x a_{t,-p}^{\prime}=a_{q q} x a_{t,-p}^{\prime}-\varepsilon_{q} \varepsilon_{t} a_{q,-t} x a_{-q,-p}^{\prime}=b_{q,-p} \in \sigma_{q,-p} .
$$

Thus $a_{t,-p}^{\prime} \in \sigma_{q,-p}$. It only remains to notice that

$$
a_{p,-t}=-\varepsilon_{p} \varepsilon_{t} a_{t,-p}^{\prime} \in \sigma_{q,-p}=\sigma_{p,-t} .
$$


Lemma 7.2. Assume $h(\nu) \geq(4,4)$. Let $(p, q, h, t, l)$ be an A-type base quintuple and a an element of $\operatorname{Sp}(2 n, R)$ such that at least one of the following three conditions holds:

1. The elements $a_{-p,-t}, a_{-h,-t}, a_{p q}, a_{h q}, a_{-p, q} a_{t,-p}^{\prime}$ and $a_{-h, q} a_{t,-h}^{\prime}$ are contained in the Jacobson radical and the entries $a_{p p}, a_{q q}$ and $a_{-t,-t}$ are invertible.

2. The rows $a_{p_{*}}, a_{q_{*}}, a_{h *}$ and the column $a_{*,-t}$ coincide modulo the Jacobson radical with the corresponding rows and columns of the identity matrix.

3. The rows $a_{-t, *}, a_{-h, *}, a_{-p, *}$ and columns $a_{* p}$ and $a_{* q}$ coincide modulo the Jacobson radical with the corresponding rows and columns of the identity matrix.

If there exist $g_{1}, g_{2} \in \operatorname{Ep}(\sigma, \Gamma)$ such that $g_{1} a g_{2} \in H$ then $a_{p,-t} \in \sigma_{p,-t}$.

Proof. Note that the first condition in the statement of this lemma trivially follows from any of the others. Consider the matrix

$$
b=a T_{p q}\left(-a_{p p}^{-1} a_{p q}\right)
$$

By the assumption that the entry $a_{p q}$ is contained in the Jacobson radical $b \equiv a \bmod J$. Clearly, $b_{p q}=0, b_{p,-t}=a_{p,-t}$ and $b_{q q}, b_{-t,-t} \in R^{*}$. Further, consider the matrix

$$
c=T_{h q}\left(-b_{h q} b_{q q}^{-1}\right) T_{-p,-t}\left(-b_{-p,-t} b_{-t,-t}^{-1}\right) T_{-h,-t}\left(-b_{-h,-t} b_{-t,-t}^{-1}\right) T_{-l,-t}\left(-b_{-l,-t} b_{-t,-t}^{-1}\right) b .
$$

As $b \equiv a \bmod J$, we have $b_{h q}, b_{-p,-t}$ and $b_{-h,-t}$ in the Jacobson radical. Therefore $c_{i *} \equiv a_{i *} \bmod J$ whenever $i \neq t,-l$ (and also $c_{* j}^{\prime} \equiv a_{* j}^{\prime} \bmod J$ whenever $j \neq l,-t$ ). In particular $c_{-p, q} c_{t,-p}^{\prime}, c_{-h, q} c_{t,-h}^{\prime} \in J$ and $c_{q q} \in R^{*}$. It is easy to see that $c_{p q}=c_{h q}=$ $c_{-p,-t}=c_{-h,-t}=c_{-l,-t}=0$. Finally, $g_{3} c g_{4} \in H$, where

$$
\begin{aligned}
g_{3}= & g_{1}\left(T_{h q}\left(-b_{h q} b_{q q}^{-1}\right) T_{-p,-t}\left(-b_{-p,-t} b_{-t,-t}^{-1}\right) \times\right. \\
& \left.T_{-q,-t}\left(-b_{-q,-t} b_{-t,-t}^{-1}\right) T_{-l,-t}\left(-b_{-l,-t} b_{-t,-t}^{-1}\right)\right)^{-1} \\
g_{4}= & T_{p q}\left(a_{p p}^{-1} a_{p q}\right) g_{2} .
\end{aligned}
$$

Clearly, $g_{3}$ and $g_{4}$ are contained in $\operatorname{Ep}(\sigma, \Gamma)$. Therefore, $c$ satisfies the conditions of Lemma 7.1 and it follows that $c_{p,-t} \in \sigma_{p,-t}$. It's only left to notice that $c_{p,-t}=a_{p,-t}$.

Lemma 7.3. Assume $h(\nu) \geq(4,4)$. Let $(p, q, h, t)$ be an A-type base quadruple and a an element of $\operatorname{Sp}(2 n, R)$ such that $a_{p,-h}, a_{q,-h}, a_{t,-h} \in \sigma_{p,-p} \cap J, a_{-h, p} \in \sigma_{-h, p} \cap J$. Suppose $a_{q p} \in J$ and $a_{p p}, a_{-h,-h} \in R^{*}$ and suppose that there exists an element $g \in \operatorname{Ep}(\sigma, \Gamma)$ such that ${ }^{g} a \in H$. Then $a_{i p} \in \sigma_{i p}$ for all $i \in I$. If additionally $a \in \operatorname{Sp}(\sigma)$ then also $S_{-h, h}\left(a^{-1}\right) \in \Gamma_{-h}$.

Proof. Consider the matrix

$$
b=T_{-h, p}\left(-a_{-h, p} a_{p p}^{-1}\right) a .
$$


As $a_{-h, p} \in J$ it follows that $b \equiv a \bmod J$. Additionally $b_{p,-h}, b_{q,-h}$ and $b_{t,-h}$ are contained in $\sigma_{p,-p}$ and $b_{-h, p}=0$. Consider the matrix

$$
c=T_{p,-h}\left(-b_{p,-h} b_{-h,-h}^{-1}\right) T_{q,-h}\left(-b_{q,-h} b_{-h,-h}^{-1}\right) T_{t,-h}\left(-b_{t,-h} b_{-h,-h}^{-1}\right) b .
$$

Again, $c \equiv a \bmod J$, in particular $c_{p p}, c_{-h,-h} \in R^{*}$. As $c$ is a symplectic matrix, it also follows that $c_{-p, h}^{\prime}=0$ and $c_{h h}^{\prime} \in R^{*}$. It's easy to see that $c_{p,-h}=c_{q,-h}=c_{t,-h}=c_{-h, p}=0$. Finally, $g g_{1} c g^{-1}$ is contained in $H$, where

$$
\begin{aligned}
g_{1}=( & T_{p,-h}\left(-b_{-h,-h}^{-1} b_{p,-h}\right) T_{q,-h}\left(-b_{-h,-h}^{-1} b_{q,-h}\right) \times \\
& \left.T_{t,-h}\left(-b_{-h,-h}^{-1} b_{t,-h}\right) T_{-h, p}\left(-a_{p p}^{-1} a_{-h, p}\right)\right)^{-1} \in \operatorname{Ep}(\sigma, \Gamma) .
\end{aligned}
$$

Pick an element $x \in S$ such that ${ }^{g} T_{p h}(x) \in H$. Applying Lemma 6.5 to the matrix $c$, the short symplectic transvection $T_{p h}(x)$ and the $A$-type base triple $(p, q, h)$, we get for all $i \neq-p,-q$ the inclusion $c_{p p}\left(c T_{p h}(x) c^{-1}\right)_{i h} \in \sigma_{i h}$. As $c_{p p}$ is invertible, we also get

$$
\delta_{i h}+c_{i p} x c_{h h}^{\prime}-\varepsilon_{p} \varepsilon_{h} c_{i,-h} x c_{-p, h}^{\prime}=\left(c T_{p h}(x) c^{-1}\right)_{i h} \in \sigma_{i h}
$$

for all $i \neq-p,-q$. We can apply Lemma 6.5 to the same matrix and transvection, but to a different A-type base triple $(p, t, h)$ and get the inclusion (39) also for $i=-q$. As $c_{h h}^{\prime}$ is invertible and $c_{-p, h}^{\prime}=0$, it follows from (39) that $c_{i p} \in \sigma_{i h}$ for all $i \neq h,-p$. Observe that $a_{i p}=c_{i p}$ for all $i \neq p, q, t,-h$. Therefore $a_{i p} \in \sigma_{i p}$ for all $i \neq p, q, t,-h,-p$. The inclusions $a_{i p} \in \sigma_{i p}$ for $i=p, q$ and $t$ are trivial and the corresponding inclusion for $i=-h$ is provided by the assumptions of the lemma. Therefore $a_{i p} \in \sigma_{i p}$ for all $i \neq-p$.

Pick an element $y \in S$ such that ${ }^{g} T_{p q}(y) \in H$ and consider the matrix $d=T_{p q}(y) a$. Clearly, it satisfies all the conditions of this lemma. Indeed, $d_{p,-h}=a_{p,-h}+y a_{q,-h} \in$ $\sigma_{p,-p} \cap J, d_{p p}=a_{p p}+y a_{q p} \in R^{*}+J \leq R^{*}$ and the rest of the entries of $d$ involved in the conditions of this lemma coincide with the corresponding entries of $a$ itself. Thus we get the inclusions $d_{i p} \in \sigma_{i p}$ for all $i \neq-p$. In particular, $d_{-q, p} \in \sigma_{-q, p}$. It's only left to notice that $d_{-q, p}=a_{-q, p}-\varepsilon_{p} \varepsilon_{q} y a_{-p, p}$ and $a_{-q, p}$ is already contained in $\sigma_{-q, p}$, while $y$ is invertible. Therefore $a_{-p, p} \in \sigma_{-p, p}$.

Assume $a \in \operatorname{Sp}(\sigma)$. By Lemma 6.5 we get the inclusion $S_{-h, h}\left(c^{-1}\right) \in \Gamma_{-h}$. As $a^{-1}=c^{-1} g_{1}^{-1}$, by Corollary 3.3 we get $S_{-h, h}\left(a^{-1}\right) \in \Gamma_{-h}$.

We combine Lemmas 7.2 and 7.3 in the following corollary.

Corollary 7.4. Assume $h(\nu) \geq(4,4)$. Let $(p, q, h, t, l)$ be an A-type base quintuple and $a$ an element of $\operatorname{Sp}(2 n, R)$. Let $I^{\prime}$ denote the set $\{p, q, h, t\}$. Suppose that $a_{i *} \equiv e_{i *}$ $\bmod J$ and $a_{*,-i} \equiv e_{*,-i} \bmod J$ whenever $i \in I^{\prime}$. Further, suppose that there exists an element $g \in \operatorname{Ep}(\sigma, \Gamma)$ such that ${ }^{g} a \in H$. Then $a_{i p} \in \sigma_{i p}$ for all $i \in I$. If additionally $a \in \operatorname{Sp}(\sigma)$ then also $S_{-p, p}\left(a^{-1}\right) \in \Gamma_{-p}$.

Proof. It's easy to see that the matrix $a$ satisfies condition (2) of Lemma 7.2, Thus we can conclude that the entries $a_{p,-h}, a_{q,-h}$ and $a_{t,-h}$ are contained in $\sigma_{p,-p}$. Moreover, the same entries are contained in the Jacobson radical by assumption. Since $a$ also satisfies the condition (3) of Corollary 7.2, it follows that $a_{-h, p}$ is contained in $\sigma_{-h, p}$. Note that 
by assumption, $a_{p p}, a_{-h,-h} \in R^{*}$ and $a_{q p} \in J$. Summing up, $a$ satisfies the conditions of Lemma 7.3. Hence $a_{i p} \in \sigma_{i p}$ for all $i \in I$. If $a \in \operatorname{Sp}(\sigma)$ then by Lemma 7.3 we get the inclusion $S_{-h, h}\left(a^{-1}\right) \in \Gamma_{-h}$. Switching the indices $p$ and $h$ in the reasoning above, we get the required inclusion $S_{-p, p}\left(a^{-1}\right) \in S_{-p, p}$.

Lemma 7.5. Assume $h(\nu) \geq(4,4)$. Let $(p, h)$ be a $C$-type base pair and a an element of $\operatorname{Sp}(2 n, R)$ such that $a_{p p} \in R^{*}$ and $a_{-h,-p} \in J$. If there exists an element $g \in \operatorname{Ep}(\sigma, \Gamma)$ such that ${ }^{g} a \in H$, then $a_{i p} \in \sigma_{i p}$ for all $i \in I$.

Proof. Consider the matrix

$$
b=T_{h p}\left(\left(-a_{h p}+1\right) a_{p p}^{-1}\right) a .
$$

Clearly, $b_{p p}=a_{p p}$ is invertible and $b_{h p}=1$. Pick using Corollary 5.4 an element $x \in S$ such that ${ }^{g} T_{p,-p}\left(x^{2}\right) \in H$. By Lemma 6.6 we get the inclusions

$$
b_{p p}\left(\delta_{i,-h}+b_{i p} x^{2} b_{-p,-h}^{\prime}\right)=b_{p p}\left(b T_{p,-p}\left(x^{2}\right) b^{-1}\right)_{i,-h} \in \sigma_{i,-h}
$$

for all $i \in I$. It's only left to notice that $b_{p p}$ and $x^{2} b_{-p,-h}^{\prime}$ are invertible and thus $b_{i p} \in \sigma_{i,-h}$ for all $i \in I$. Finally, $a_{i p}=b_{i p}$ whenever $i \neq h,-p$. Thus $a_{i p} \in \sigma_{i p}$.

The following corollary is an illustration of application of Corollary 7.4 and Lemma 7.5. Suppose $R^{\prime}=R$ and $S=\{1\}$. Then it is clear that $\left(\sigma^{\prime}, \Gamma^{\prime}\right)=(\sigma, \Gamma)$ is the net associated with $H$ in $\operatorname{Sp}(2 n, R)$. Corollary 7.4 together with Lemma 7.5 yield the following result.

Corollary 7.6. Let $R$ be a commutative associative unital ring with Jacobson radical $J$. Let $\nu$ be a unitary equivalence relation on the index set $I$ such that $h(\nu) \geq(4,5)$. Let $H$ be a subgroup of $\operatorname{Sp}(2 n, R)$ such that $\operatorname{Ep}(\nu, R) \leq H$ and let $(\sigma, \Gamma)$ be the form net associated with $H$. Then

$$
H \cap \operatorname{Sp}(2 n, R, J) \leq \operatorname{Sp}(\sigma, \Gamma),
$$

where $\operatorname{Sp}(2 n, R, J)$ denotes the principal congruence subgroup of $\operatorname{Sp}(2 n, R)$ of level $J$.

\section{Over a local ring}

In this section we will prove our main result, Theorem 1 for the case when the ground ring $R$ is local. Moreover, we will prove a version of this result suitable for application in localization based proof of the next chapter. Throughout this section fix a standard setting $\left(R, R^{\prime}, S\right)$, where $R$ is a commutative local ring. Let $J$ denote the Jacobson radical of $R$ (which is the only maximal ideal of $R$ ). Further, fix a unitary equivalence relation $\nu$ on the index set $I$, a subgroup $H$ of $\operatorname{Sp}(2 n, R)$ and an exact major form net of ideals $\left(\sigma^{\prime}, \Gamma^{\prime}\right)$ which is $S$-associated with $H$. Let $(\sigma, \Gamma)$ denote the $S$-closure of the $\left(\sigma^{\prime}, \Gamma^{\prime}\right)$ in $R$. In this section we will show that

$$
H \leq \operatorname{Transp}_{\operatorname{Sp}(2 n, R)}\left(\operatorname{Ep}\left(\sigma^{\prime}, \Gamma^{\prime}\right), \operatorname{Sp}(\sigma, \Gamma)\right)
$$

provided $h(\nu) \geq(4,5)$. 
Theorem 8.1. Let $\left(R, R^{\prime}, S\right), \nu, H,\left(\sigma^{\prime}, \Gamma^{\prime}\right)$ and $(\sigma, \Gamma)$ be as above. Assume $h(\nu) \geq(4,5)$. Let a be an element of $H$ and $T_{s r}(\xi)$ be a $\left(\sigma^{\prime}, \Gamma^{\prime}\right)$-elementary transvection (long or short). Then $a T_{s r}(\xi) a^{-1} \in \operatorname{Sp}(\sigma, \Gamma)$. In other words,

$$
H \leq \operatorname{Transp}_{S p(2 n, R)}\left(\operatorname{Ep}\left(\sigma^{\prime}, \Gamma^{\prime}\right), \operatorname{Sp}(\sigma, \Gamma)\right) .
$$

Proof. As $h(\nu) \geq(4,5)$ every index $p \in I$ embeds either into an A-type base quintuple or into a C-type base pair. Each of these two cases fork one more time depending on whether $T_{s r}(\xi)$ is long or short. We consider all four options separately in form of Lemmas 8.2 , 8.3, 8.4 and 8.5 .

Now apply Theorem 8.1 to a trivial standard setting, namely $R=R^{\prime}$ and $S=\{1\}$. Togehter with Lemma 4.1 this yields Theorem 1 once the ground ring $R$ is local.

Lemma 8.2. Assume $h(\nu) \geq(4,4)$. Let a be an element of $\operatorname{Sp}(2 n, R)$ and $T_{s r}(\xi)$ a short $\left(\sigma^{\prime}, \Gamma^{\prime}\right)$-elementary transvection. Suppose that there exists an element $g \in \operatorname{Ep}(\sigma, \Gamma)$ such that $g a \in H$. Let $b$ denote the short root element $a T_{s r}(\xi) a^{-1}$. If $(p, h)$ is a $\mathrm{C}$-type base pair then for all $i \in I$ the inclusion

$$
b_{i p} \in \sigma_{i p}
$$

holds.

Proof. We will organize the analysis into seven steps.

1. Assume that the elements $a_{-p,-r}, a_{p,-r}$ and one of the element $a_{-h,-r}$ or $a_{h,-r}$ is contained in $J$. Without loss of generality, we may assume that $a_{-h,-r} \in J$. In this case it's easy to see that $b_{p p}$ is invertible and $b_{-h,-p} \in J$. Indeed,

$$
\begin{aligned}
b_{p p} & =1+a_{p s} \xi a_{r p}^{\prime}-\varepsilon_{s} \varepsilon_{r} a_{p,-r} \xi a_{-s, p}^{\prime} \in 1+J \leq R^{*}, \\
b_{-h,-p} & =a_{-h, s} \xi a_{r,-p}^{\prime}-\varepsilon_{s} \varepsilon_{r} a_{-h,-r} \xi a_{-s,-p}^{\prime} \in J .
\end{aligned}
$$

Therefore, the matrix $b$ satisfies the conditions of Lemma 7.5 and we get the inclusions $b_{i p} \in \sigma_{i p}$ for all $i \in I$.

2. Assume that $a_{-p,-r} \in J$, but either $b_{h,-r} \in R^{*}$ or $b_{-h,-r} \in R^{*}$. We can assume that $a_{h,-r} \in R^{*}$, otherwise switch $h$ and $-h$. Consider the matrices

$$
c=T_{p h}\left(-a_{p,-r} a_{h,-r}^{-1}\right) a \quad d=T_{-h, h}\left(-c_{-h,-r} b_{h,-r}^{-1}\right) d .
$$

It's easy to see that $d_{p,-r}=d_{-h,-r}=0$ and by definition $g_{1} d=a \in H$, where $g_{1} \in$ $\operatorname{Ep}(\sigma, \Gamma)$. Finally, $d_{-p,-r}=a_{-p,-r} \in J$. By the previous step we get the inclusion $f_{i p} \in \sigma_{i p}$ for all $i \in I$, where $f=d T_{s r}(\xi) d^{-1}$. It's only left to notice that $b_{i p}=f_{i p}$ whenever $i \neq p,-h$ and the rest of the required inclusions are trivial.

3. Assume that $a_{-p,-r}, a_{h,-r}, a_{-h,-r} \in J$, but $a_{p,-r} \in R^{*}$. Then by step 1 we get $b_{i h} \in \sigma_{i h}$ for all $i \in I$. Now consider the matrix

$$
c=T_{h p}(1) a, \quad f=c T_{s r}(\xi) c^{-1} .
$$


Observe that $c_{h,-r} \in R^{*}$ and $c_{-p,-r}=a_{-p,-r} \pm a_{-h,-r} \in J$. By step 2 we get $f_{i p} \in \sigma_{i p}$ for all $i \in I$. Finally, $f_{i p}=b_{i p}+b_{i h}$ whenever $i \neq h,-p$ Therefore $b_{i p} \in \sigma_{i p}$ for all $i \in I$.

Steps 1-3 deal prove the lemma once $a_{-p,-r} \in J$. Note that as $T_{s r}(\xi)=T_{-r,-s}(\xi)$, we can replace $r$ by $-s$ and $s$ by $-r$ in all the reasonings in steps 1-3. We can sum up the results of cases 1-3 together with the last observation as follows.

4. Assume that $a_{-p,-r} \in J$ or $a_{-p, s} \in J$. Then $b_{i p} \in \sigma_{i p}$ for all $i \in I$.

Before we continue with the opposite assumption, we will establish another version of step 1.

5. Assume $a_{p s}, a_{p,-r}, a_{-h,-r} \in J$. Then as in case 1 we get

$$
\begin{aligned}
b_{p p} & =1+a_{p s} \xi a_{r p}^{\prime}-\varepsilon_{s} \varepsilon_{r} a_{p,-r} \xi a_{-s, p}^{\prime} \in 1+J \leq R^{*}, \\
b_{-h,-p} & =a_{-h, s} \xi a_{r,-p}^{\prime}-\varepsilon_{s} \varepsilon_{r} a_{-h,-r} \xi a_{-s, p}^{\prime}= \pm a_{-h, s} \xi a_{p,-r} \pm a_{-h,-r} \xi a_{-p s} \in J .
\end{aligned}
$$

Thus by Lemma 7.5 we get the inclusions $b_{i p} \in \sigma_{i p}$ for all $i \in I$.

The last two steps deal with the situation when $a_{-p,-r} \in R^{*}$.

6. Assume that $a_{-p,-r}, a_{-p, s}, a_{p s} \in R^{*}$ and $a_{p,-r} \in J$. By case 4 we have $b_{i,-p} \in \sigma_{i,-p}=$ $\sigma_{i p}$ for all $i \in I$. Consider the matrices

$$
c=T_{-p, p}\left(-a_{-p, s} a_{p s}^{-1}\right) a, \quad f=c T_{s r}(\xi) c^{-1} .
$$

Clearly, $c_{-p, s}=0$ and it follows by case 4 that $f_{i p} \in \sigma_{i p}$ for all $i \in I$. Now observe that $f_{i p}=b_{i p}-a_{-p, s} a_{p s}^{-1} b_{i,-p}$ for all $i \neq-p$. As $b_{i,-p} \in \sigma_{i,-p}$ we get $b_{i p} \in \sigma_{i p}$ for all $i \in I$.

7. Finally, assume that $a_{-p,-r}, a_{-p, s} \in R^{*}$. Consider the matrices

$$
c=T_{-h,-p}\left(-a_{-h,-r} a_{-p,-r}^{-1}\right) a, \quad d=T_{p,-p}\left(-b_{p,-r} a_{-p,-r}^{-1}\right) b, \quad f=d T_{s r}(\xi) c^{-1} .
$$

It's easy to see that $d_{-h,-r}=0$ and $d_{p,-r}=0$. Further $d_{-p, s}=a_{-p, s} \in R^{*}$ and $d_{-p,-r}=$ $a_{-p,-r} \in R^{*}$. If $d_{p s} \in J$ then by step 5 we get $f_{i p} \in \sigma_{i p}$ for all $i \in I$. On the other hand if $d_{p s} \in R^{*}$ then by step 6 we have again $f_{i p} \in \sigma_{i p}$. It's only left to notice that $b_{i p}=f_{i p}$ whenever $i \neq-h, p$.

In order to complete the proof note that the assumptions of steps 4 and 7 exhaust all the possibilities.

Lemma 8.3. Assume $h(\nu) \geq(4,4)$. Let a be an element of $S p(2 n, R)$ such that ga $\in H$ for some $g \in \operatorname{Ep}(\sigma, \Gamma)$. Let $T_{s,-s}(\alpha)$ be a long $\left(\sigma^{\prime}, \Gamma^{\prime}\right)$-elementary transvection and $b$ the long root element $a T_{s,-s}(\alpha) a^{-1}$. If $(p, h)$ is a $\mathrm{C}$-type base pair then for all $i \in I$ the inclusion

$$
b_{i p} \in \sigma_{i p}
$$

holds.

Proof. We will organize the analysis into four cases.

1. Assume that $a_{p s} \in J$. Then it's easy to see that $b_{p p}$ is invertible and $b_{-h,-p} \in J$. Indeed,

$$
\begin{aligned}
b_{p p} & =1+a_{p s} \alpha a_{-s, p}^{\prime} \in 1+J \leq R^{*}, \\
b_{-h,-p} & =a_{-h, s} \alpha a_{-s,-p}^{\prime}= \pm a_{-h, s} \alpha a_{p s} \in J .
\end{aligned}
$$


Therefore, the matrix $b$ satisfies the conditions of Lemma 7.5 and we get the inclusions $b_{i p} \in \sigma_{i p}$ for all $i \in I$.

2. Assume that $a_{h s}$ or $a_{-h, s}$ is invertible. Without loss of generality, assume the first, otherwise, simply switch $h$ and $-h$. Consider the matrix

$$
c=T_{p h}\left(-a_{p s} a_{h s}^{-1}\right) a .
$$

Clearly, $c_{p s}=0 \in J$. Put $f=c T_{s,-s}(\alpha) c^{-1}$. We can apply the first case of this lemma to the pair of matrices $c$ and $f$ in place of $a$ and $b$ and obtain the inclusions $f_{i p} \in \sigma_{i p}$ for all $i \in I$. It's only left to notice that $b_{i p}=f_{i p} \in \sigma_{i p}$ whenever $i \neq p,-h$ and the rest of the required inclusions are trivial as the form net $(\sigma, \Gamma)$ is major.

3. Finally, assume that $a_{p s}$ is invertible, but $a_{h s} \in J$ and $a_{-h, s} \in J$. Switching $p$ and $h$ in the second case of this lemma we get $b_{i h} \in \sigma_{i h}=\sigma_{i p}$ for all $i \in I$. Consider

$$
c=T_{p h}(1) a, \quad f=c T_{s,-s}(\alpha) c^{-1} .
$$

Clearly, $c_{p s}=a_{p s}+a_{h s} \in R^{*}+J \in R^{*}$ and $c_{h s}=a_{h s} \in J$. Thus, again by the second case we get $f_{i h} \in \sigma_{i p}$ for all $i \in I$. Finally, it's easy to see that $f_{i h}=b_{i h}+b_{i p}$ for all $i \neq p,-h$. As we already know that $b_{i h} \in \sigma_{i p}$. As $(\sigma, \Gamma)$ is major, this yields the inclusion $b_{i p} \in \sigma_{i p}$ for all $i \in I$.

Lemma 8.4. Assume $h(\nu) \geq(4,4)$. Let a be an element of $\operatorname{Sp}(2 n, R)$ such that $g_{1} a \in H$ for some $g_{1} \in \operatorname{Ep}(\sigma, \Gamma)$. Let $T_{s r}(\xi)$ be a short $\left(\sigma^{\prime}, \Gamma^{\prime}\right)$-elementary transvection and $b$ the short root element $a T_{s r}(\xi) a^{-1}$. Let $(p, q, h, t, l)$ be an A-type base quintuple. Then the inclusion

$$
b_{i p} \in \sigma_{i p}
$$

holds for any $i \in I$. If additionally $b \in \operatorname{Sp}(\sigma)$ then also

$$
S_{-p, p}\left(b^{-1}\right) \in \Gamma_{-p}
$$

Proof. Denote by $I^{\prime}$ the set $\{p, q, h, t, l\}$. This proof is organized as follows.

1. We will show that if $a_{l,-r}$ is invertible then the inclusion (40) holds for any $i \in I$ and if additionally $b \in \operatorname{Sp}(\sigma)$ then also (41) holds.

2. We will show that if there exists an index $i \in I^{\prime}$ such that $a_{i,-r}$ or $a_{i s}$ is invertible then the inclusion (40) holds for any $i \in I$ and if additionally $b \in \operatorname{Sp}(\sigma)$ then also (41) holds. This case can be reduced to the previous one.

3. Finally, if $a_{i,-r}, a_{i s} \in J$ for all $i \in I^{\prime}$ then the required inclusions (40) and (41) follow from Corollary 7.4 .

1. Suppose $a_{l,-r} \in R^{*}$. Let

$$
\begin{aligned}
g & =T_{t l}\left(a_{t,-r} a_{l,-r}^{-1}\right) T_{h l}\left(a_{h,-r} a_{l,-r}^{-1}\right) T_{q l}\left(a_{q,-r} a_{l,-r}^{-1}\right) T_{p l}\left(a_{p,-r} a_{l,-r}^{-1}\right), \\
c & =g^{-1} a \text { and } \\
d & =c T_{s r}(\xi) c^{-1}={ }^{-1} b .
\end{aligned}
$$


It is easy to see that $c_{p,-r}=c_{q,-r}=c_{h,-r}=c_{t,-r}=0$ and if $b \in \operatorname{Sp}(\sigma)$ then also $d \in \operatorname{Sp}(\sigma)$. We will consider three subcases:

i. There is an index $i_{1} \in I^{\prime} \backslash\{p, l\}$ such that $c_{i_{1}, s}$ is invertible.

ii. The only $i_{1} \in I^{\prime} \backslash\{l\}$ such that $c_{i_{1}, s} \in R^{*}$ is $i_{1}=p$.

iii. $c_{i s} \in J$ for all $i \in I^{\prime} \backslash\{l\}$.

For each of the cases (1.i)-(1.iii) we will prove that $d_{i p} \in \sigma_{i p}$ for all $i \in I$ and if $b \in \operatorname{Sp}(\sigma)$ then $S_{-p, p}\left(d^{-1}\right) \in \Gamma_{-p}$. Note that $d_{i p}=b_{i p}$ for all $i \neq p, q, h, t,-l$, the inclusion $b_{i p} \in \sigma_{i p}$ for $i=p, q, h, t$ is trivial and

$$
d_{-l, p}=b_{-l, p} \pm \zeta_{p} b_{-p, p} \pm \zeta_{q} b_{-q, p} \pm \zeta_{h} b_{-h, p} \pm \zeta_{t} b_{-t, p},
$$

where $\zeta_{i} \in R$. As $b_{-i, p} \in \sigma_{-p, p}$ for $i=p, q, t, h$ it follows that $b_{-l, p} \in \sigma_{-l, p}$. Summing up, $b_{i p} \in \sigma_{i p}$ for all $i \in I$.

Assume that $b \in \operatorname{Sp}(\sigma)$. By Corollary 3.3 we get

$$
S_{-p, p}\left(b^{-1}\right) \equiv S_{-p, p}\left(g^{-1} b^{-1}\right) \equiv S_{-p, p}\left(d^{-1}\right) \quad \bmod \Gamma_{-p} .
$$

Hence, $S_{-p, p}\left(b^{-1}\right) \in \Gamma_{-p}$. This completes the analysis of the case 1 . Now we consider the cases (1.i)-(1.iii).

1.i. Suppose there exists an index $i_{1} \in I^{\prime} \backslash\{p, l\}$ such that $c_{i_{1}, s} \in R^{*}$. In this situation we can apply Lemma 6.5 to the matrix $c$, a short $\left(\sigma^{\prime}, \Gamma^{\prime}\right)$-elementary transvection $T_{s r}(\xi)$ and the A-type base triple $\left(i_{1}, i_{2}, p\right)$, where $i_{2}$ can be chosen in $I^{\prime} \backslash\left\{p, l, i_{1}\right\}$. Thus we get $c_{i_{1}, s} d_{i p} \in \sigma_{i p}$ for all $i \neq-i_{1}$ and if $b \in \operatorname{Sp}(\sigma)$ then also $c_{i_{1}, s}^{2} S_{-p, p}\left(d^{-1}\right) \in$ $\Gamma_{-p}$. As $c_{i_{1}, s}$ is invertible, it follows that $d_{i p} \in \sigma_{i p}$ for all $i \neq-i_{1}$ and if $b \in \operatorname{Sp}(\sigma)$ then $S_{-p, p}\left(d^{-1}\right) \in \Gamma_{-p}$.

Pick an index $i_{2} \in I^{\prime} \backslash\left\{p, l, i_{1}\right\}$. If $c_{i_{2}, s}$ is invertible in $R$ we can replace $i_{1}$ in the reasoning above with $i_{2}$ and get the missing inclusion $d_{-i_{1}, p} \in \sigma_{-i_{1}, p}$. If $c_{i_{2}, s}$ is not invertible, consider the matrices $f=T_{i_{1}, i_{2}}(1) c$ and $g=f T_{s r}(\xi) f^{-1}={ }^{T_{i_{1}, i_{2}}(1)} d$. Clearly $f_{p,-r}=f_{q,-r}=f_{h,-r}=f_{t,-r}=0$ and $f_{i_{1}, s}, f_{i_{2}, s} \in R^{*}$. Moreover ${ }^{g T_{i_{1}, i_{2}}(-1)} b \in$ $H$. Therefore we deduce by Lemma 6.5 that $f_{i_{1}, s} g_{-i_{2}, p} \in \sigma_{-p, p}$ and thus $g_{-i_{2}, p} \in$ $\sigma_{-p, p}$. Finally $g_{-i_{2}, p}=d_{-i_{2}, p}+d_{-i_{1}, p}$ and, as $d_{-i_{2}, p} \in \sigma_{-p, p}$, it follows also that $d_{-i_{1}, p} \in \sigma_{-p, p}$. Therefore $d_{i p} \in \sigma_{i p}$ for all $i \in I$ and if $b \in \operatorname{Sp}(\sigma)$ then also $S_{-p, p}\left(d^{-1}\right) \in \Gamma_{-p}$.

1.ii. Suppose $c_{h s}, c_{q s}, c_{t s} \in J$, but $c_{p s} \in R^{*}$. By the case (1.i) the inclusion $d_{i h} \in \sigma_{i h}$ holds for any $i \in I$ and if $b \in \operatorname{Sp}(\sigma)$ then also $S_{-h, h}\left(d^{-1}\right) \in \Gamma_{-h}$. Consider the matrices $f=T_{h p}(1) c$ and $g={ }^{T_{h p}(1)} d$. Then ${ }^{g T_{h p}(-1)} b \in H, f_{p,-r}=f_{q,-r}=f_{h,-r}=f_{t,-r}=0$ and $f_{h s} \in R^{*}$. By case (1.i) we get $g_{i p} \in \sigma_{i p}$ for all $i \in I$ and if $b \in \operatorname{Sp}(\sigma)$ then also $S_{-p, p}\left(g^{-1}\right) \in \Gamma_{-p}$. Observe that $g_{i p}=d_{i p}+d_{i h}$ for all $i \neq h,-p$ and, as $d_{i h}$ is already contained in $\sigma_{i p}$ for all $i \in I$, we get $d_{i p} \in \sigma_{i p}$ for all $i \neq-p$. Finally

$$
g_{-p, p}=d_{-p, p} \pm d_{-h, p} \pm d_{-p, h} \pm d_{-h, h}
$$


and, as $d_{-h, p}= \pm d_{-p, h}^{\prime}= \pm d_{-p, h}$, the last three summands in (42) are contained in $\sigma_{-p, p}$. Thus $d_{-p, p} \in \sigma_{-p, p}$. If $b \in \operatorname{Sp}(\sigma)$ then by Corollary 3.3 we have

$$
S_{-p, p}\left(g^{-1}\right)=S_{-p, p}\left({ }^{T_{h p}(1)} d^{-1}\right) \equiv S_{-p, p}\left(d^{-1}\right)+S_{-h, h}\left(d^{-1}\right) \quad \bmod \Gamma_{-p} .
$$

As $S_{-h, h}\left(d^{-1}\right) \in \Gamma_{-p}$, we get $S_{-p, p}\left(d^{-1}\right) \in \Gamma_{-p}$.

1.iii. Suppose $c_{i s}, c_{i,-r} \in J$ for all $i \in I^{\prime} \backslash\{l\}$. Then $b_{i j} \equiv \delta_{i j} \bmod J$ whenever $i \in I^{\prime} \backslash\{l\}$ or $-j \in I^{\prime} \backslash\{l\}$. By Corollary 7.4 we get the required inclusions $d_{i p} \in \sigma_{i p}$ for all $i \in I$ and if $b \in \operatorname{Sp}(\sigma)$ then also $S_{-p, p}\left(d^{-1}\right) \in \Gamma_{-p}$.

2. Suppose $a_{l,-r} \in J$, but there still exists an index $i_{1} \in I^{\prime}$ such that $a_{i_{1},-r} \in R^{*}$ or $a_{i_{1}, s} \in R^{*}$. First, assume $a_{i_{1},-r} \in R^{*}$. By case 1 we have $b_{i l} \in \sigma_{i l}$ for all $i \in I$ and if $b \in \operatorname{Sp}(\sigma)$ then also $S_{-l, l}\left(b^{-1}\right) \in \Gamma_{-l}$. Consider the matrices $c=T_{l, i_{1}}(1) a \in H$ and $d={ }^{T_{l, i_{1}}(1)} b$. Then $c_{l,-r} \in R^{*}$ and by case 1 we get $d_{i p} \in \sigma_{i p}$ and if $b \in \operatorname{Sp}(\sigma)$ then also $S_{-p, p}\left(d^{-1}\right) \in \Gamma_{-p}$. Note that if $a \in \operatorname{Sp}(\sigma)$ we have

$$
S_{-p, p}\left(d^{-1}\right) \equiv S_{-p, p}\left(T_{l, i_{1}}(1) b^{-1}\right) \equiv S_{-p, p}\left(b^{-1}\right)+\delta_{p, i_{1}} S_{-l, l}\left(b^{-1}\right) \quad \bmod \Gamma_{-p} .
$$

Recall that $S_{-l, l}\left(b^{-1}\right) \in \Gamma_{-l}=\Gamma_{-p}$. Therefore $S_{-p, p}\left(b^{-1}\right) \in \Gamma_{-p}$. As $d_{i p}=b_{i p}+$ $\delta_{i_{1}, p} b_{i l}$ for all $i \neq l,-i_{1}$, it follows that $b_{i p} \in \sigma_{i p}$ for all $i \neq-i_{1}$. Finally we have

$$
d_{-i_{1}, p}=b_{-i_{1}, p} \pm b_{-l, p}+\delta_{i_{1}, p} b_{-i_{1}, l} \pm \delta_{i_{1}, p} b_{-l, l} .
$$

Note that $b_{-l, p}= \pm b_{-p, l}$. Thus, the last three summands in (43) are contained in $\sigma_{-p, p}$. Therefore $b_{-i_{1}, p} \in \sigma_{-p, p}$. We conclude that $b_{i p} \in \sigma_{i p}$ for all $i \in I$ and if $b \in \operatorname{Sp}(\sigma)$ then also $S_{-p, p}\left(b^{-1}\right) \in \Gamma_{-p}$.

Finally, if $a_{i_{1}, s} \in R^{*}$ we can use the Steinberg relation (R1), namely $T_{s r}(\xi)=$ $T_{-r,-s}( \pm \xi)$. Set $d=a T_{-r,-s}(\xi) a^{-1}$. We have already shown that in this case $d_{i p} \in \sigma_{i p}$ for all $i \in I$ and $S_{-p, p}\left(d^{-1}\right) \in \Gamma_{-p}$. Finally $b_{i p}= \pm d_{i p}$ for all $i \neq p$ and if $b \in \operatorname{Sp}(\sigma)$ then $S_{-p, p}\left(d^{-1}\right)=S_{-p, p}\left(b^{-1}\right) \pm 2 b_{-p, p}\left(2-b_{-p,-p}\right) \equiv S_{-p, p}\left(b^{-1}\right) \bmod \Gamma_{-p}$. Therefore $S_{-p, p}\left(b^{-1}\right) \in \Gamma_{-p}$.

3. Suppose $a_{i s}, a_{i,-r} \in J$ for all $i \in I^{\prime}$. Exactly as in case (1.iii) we get all the required inclusions via Corollary 7.4 .

This completes the proof.

Lemma 8.5. Assume $h(\nu) \geq(4,4)$. Let a be an element of $\operatorname{Sp}(2 n, R)$ such that $g a \in H$ for some $g \in \operatorname{Ep}(\sigma, \Gamma)$. Let $T_{s,-s}(\alpha)$ be a long $\left(\sigma^{\prime}, \Gamma^{\prime}\right)$-elementary transvection and $b$ the long root element $a T_{s,-s}(\alpha) a^{-1}$. Let $(p, q, h, t, l)$ be an $\mathrm{A}$-type base quintuple. Then the inclusion

$$
b_{i p} \in \sigma_{i p}
$$

holds for any $i \in I$. If additionally $b \in \operatorname{Sp}(\sigma)$ then also

$$
S_{-p, p}\left(b^{-1}\right) \in \Gamma_{-p}
$$


Proof. The proof is based on application of Corollary 7.4. Recall that

$$
b_{i j}=\delta_{i j}+a_{i s} \alpha a_{-s, j}^{\prime}=\delta_{i j} \pm a_{i s} \alpha a_{-j, s}
$$

Thus

$$
b_{i j} \equiv \delta_{i j} \text { whenever } a_{i s} \in J \text { or } a_{-j, s} \in J \text {. }
$$

Set $I^{\prime}=\{p, q, h, t, l\}$.

1. First we will show that once there exists an index $j$ in the set $I^{\prime} \backslash\{p\}$ such that $a_{j s}$ is invertible we can make sure that $b$ satisfies the conditions of Corollary 17.4, maybe after multiplying $a$ on the left with an element of $\operatorname{Ep}(\sigma, \Gamma)$. Without loss of generality, assume that $j=l$. Consider the matrices

$$
\begin{aligned}
g_{1} & =T_{p l}\left(-a_{p s} a_{l s}^{-1}\right) T_{q l}\left(-a_{q s} a_{l s}^{-1}\right) T_{h l}\left(-a_{h s} a_{l s}^{-1}\right) T_{t l}\left(-a_{t s} a_{l s}^{-1}\right), \\
c & =g_{1} a, \\
f & =c T_{s,-s}(\alpha) c^{-1}=g_{1} b g_{1}^{-1} .
\end{aligned}
$$

Clearly, $a_{p s}=a_{q s}=a_{t s}=a_{h s}=0 \in J$. This yields by (46) that $b_{i j} \equiv \delta_{i j} \bmod J$ whenever $i \in I^{\prime} \backslash\{l\}$ or $-j \in I^{\prime} \backslash\{l\}$. It follows by Corollary 7.4 that $f_{i p} \in \sigma_{i p}$ for all $i \in I$. Further, if $b \in \operatorname{Sp}(\sigma)$ then $f \in \operatorname{Sp}(\sigma)$ and by Corollary 7.4 also $S_{-p, p}\left(f^{-1}\right) \in \Gamma_{-p}$. Finally, using Corollary 3.3 we infer that $S_{-p, p}\left(b^{-1}\right)=S_{-p, p}\left(g_{1}^{-1} f^{-1} g_{1}\right) \equiv S_{-p, p}\left(f^{-1}\right)$ $\bmod \Gamma_{-p}$. Therefore $S_{-p, p}\left(b^{-1}\right) \in \Gamma_{-p}$.

2. Next, assume that $a_{p s}$ is invertible, but $a_{q s}, a_{h s}, a_{t s}$ and $a_{l s}$ are contained in $J$. It follows by case 1 that $b_{i l} \in \sigma_{i l}$ and if $b \in \operatorname{Sp}(\sigma)$ then $S_{-l, l}\left(b^{-1}\right) \in \Gamma_{-p}$. Consider the matrices

$$
c=T_{l p}(1) a, \quad f=c T_{s,-s}(\alpha) c^{-1}={ }^{T_{l p}(1)} b .
$$

Clearly, $c_{l s}=a_{l s}+a_{p s} \in R^{*}$ and we can apply case 1 to the matrices $c$ and $f$ instead of $a$ and $b$. Thus we get $c_{i p} \in \sigma_{i p}$ for all $i \in I$ and if $b \in \operatorname{Sp}(\sigma)$ (and thus also $f \in \operatorname{Sp}(\sigma)$ ) then also $S_{-p, p}\left(f^{-1}\right) \in \Gamma_{-p}$. It's only left to notice that $f_{i p}=b_{i p}+b_{i l}$ and by Corollary 3.3 we have $S_{-p, p}\left(f^{-1}\right)=S_{-p, p}\left(T_{l p}^{-1}(1) b^{-1} T_{l p}(1)\right) \equiv S_{-p, p}\left(b^{-1}\right)+S_{-l, l}\left(b^{-1}\right)$ whenever $b \in \operatorname{Sp}(\sigma)$. We have already noticed that $b_{i l} \in \sigma_{i l}$ for all $i \in I$, therefore also $b_{i p} \in \sigma_{i p}$. If $b \in \operatorname{Sp}(\sigma)$ then, as $S_{-l, l}\left(b^{-1}\right), S_{-p, p}\left(f^{-1}\right) \in \Gamma_{-p}$, also $S_{-p, p}\left(b^{-1}\right) \in \Gamma_{-p}$.

3. Finally, if $a_{i s} \in J$ for all $i \in I^{\prime}$ then it follows by (46) that $b_{i j} \equiv \delta_{i j} \bmod J$ whenever $i \in I^{\prime}$ or $-j \in I^{\prime}$. This yields by Corollary 7.4 the inclusion (44) for all $i \in I$. If $b \in \operatorname{Sp}(\sigma)$ then for the same reason we get the inclusion (45).

\section{Localization}

In this section we will prove Theorem 1 for any commutative ring. We will first show that it's enough to prove our theorem for a Noetherian ground ring. The general result follows almost immediately from the Noetherian case. For the case of a Noetherian ground ring we use the following scheme to prove that $H \leq \operatorname{Transp}_{\operatorname{Sp}(2 n, R)}(\operatorname{Ep}(\sigma, \Gamma), \operatorname{Sp}(\sigma, \Gamma)$, where $(\sigma, \Gamma)$ is the form net of ideals over $R$, associated with $H$ (cf. Lemma 4.1 of Section 4). First, pick a maximal ideal $\mathfrak{m}$ of $R$ and consider the localization $R_{\mathfrak{m}}=(R \backslash \mathfrak{m})^{-1} R$ of 
the ring $R$. This is a local ring. More importnantly, there exists an element $x_{0} \in R \backslash \mathfrak{m}$ such that the homomorphism $\mathrm{M}\left(F_{\mathfrak{m}}\right)$ induced by the localization morphism $F_{\mathfrak{m}}: R \longrightarrow$ $R_{\mathfrak{m}}, \xi \mapsto \frac{\xi}{1}$ is injective on $\operatorname{Sp}\left(2 n, R, x_{0} R\right)$. This allows us to push the subgroup $H$ together with the form net of ideals $(\sigma, \Gamma)$ into the ring $R_{\mathfrak{m}}$, use Theorem 8.1 there (observe that $\left(R_{\mathfrak{m}}, F_{\mathfrak{m}}(R), F_{\mathfrak{m}}(R \backslash \mathfrak{m})\right)$ becomes a standard setting and the cooridinate-wise image of $(\sigma, \Gamma)$ under $F_{\mathfrak{m}}$ becomes a form net $F_{\mathfrak{m}}(R \backslash \mathfrak{m})$-associated with $\left.\mathrm{M}\left(F_{\mathfrak{m}}\right)(H)\right)$ and pull the resulting inclusion back to the ring $R$ using injectivity of $M\left(F_{\mathfrak{m}}\right)$ and the idea of patching, which amounts to being able to get the unity of a ring as a linear combination of elements of any subset of this ring which is unimodular, i.e. not contained in any maximal ideal. The uniqueness part of Theorem 1 follows trivially from Theorem 2 ,

\section{Noetherian reduction.}

Suppose Theorem 1 holds for any Noetherian ground ring. It is a well known fact that every commutative ring $R$ is a direct $\operatorname{limit} \lim R^{\prime}$ of its Noetherian subrings $R^{\prime}$. Fix a commutative ring $R$, a unitary equivalence relation $\nu$ such that $h(\nu) \geq(4,5)$ and a subgroup $H$ such that $\operatorname{Ep}(\nu, R) \leq H$. Let $(\sigma, \Gamma)$ denote the exact major form net of ideals associated with $H$, cf. Lemma 4.1, For any Noetherian subring $R^{\prime}$ of $R$ set $H^{\prime}=H \cap \operatorname{Sp}\left(2 n, R^{\prime}\right)$. Then $\operatorname{Ep}\left(\nu, R^{\prime}\right) \leq \operatorname{Ep}(\nu, R) \cap \operatorname{Sp}\left(2 n, R^{\prime}\right) \leq H^{\prime}$. By Lemma 4.1 there exists an exact major form net of ideals $\left(\sigma^{\prime}, \Gamma^{\prime}\right)$ over $R^{\prime}$ associated with $H^{\prime}$. By the construction in Lemma 4.1 of a form net associated with a subgroup it follows that if $R^{\prime} \leq R^{\prime \prime}$ then $\left(\sigma^{\prime}, \Gamma^{\prime}\right) \leq\left(\sigma^{\prime \prime}, \Gamma^{\prime \prime}\right)$. Clearly $\operatorname{Sp}\left(\sigma^{\prime}, \Gamma^{\prime}\right) \leq \operatorname{Sp}\left(\sigma^{\prime \prime}, \Gamma^{\prime \prime}\right)$. As any element $g$ of $\operatorname{Sp}(2 n, R)$ is contained in $\operatorname{Sp}\left(2 n, R^{\prime \prime}\right)$ for some Noetherian subring $R^{\prime \prime}$ of $R$ such that $R^{\prime} \leq R^{\prime \prime}$, it follows that

$$
\operatorname{Sp}(\sigma, \Gamma)=\underset{R^{\prime} \text { is Noetherian }}{\lim _{\text {Non }}} \operatorname{Sp}\left(\sigma^{\prime}, \Gamma^{\prime}\right) .
$$

Pick any $a \in H$ and $T_{s r}(\xi) \in \operatorname{Ep}(\sigma, \Gamma)$. There exists a Noetherian subring $R^{\prime}$ of $R$ such that $a, T_{s r}(\xi) \in \operatorname{Sp}\left(2 n, R^{\prime}\right)$. Clearly, $a \in H^{\prime}$ and $T_{s r}(\xi) \in \operatorname{Ep}\left(\sigma^{\prime}, \Gamma^{\prime}\right)$. By assumption, Theorem 1 holds for the ground ring $R^{\prime}$. Therefore

$$
a T_{s r}(\xi) a^{-1} \in \operatorname{Sp}\left(\sigma^{\prime}, \Gamma^{\prime}\right)
$$

Moreover the inclusion (48) holds for any Noetherian subring $R^{\prime \prime}$ such that $R^{\prime} \leq R^{\prime \prime}$. Combining (48) with (47) we deduce that

$$
\operatorname{Ep}(\sigma, \Gamma) \leq H \leq \operatorname{Transp}_{\operatorname{Sp}(2 n, R)}(\operatorname{Ep}(\sigma, \Gamma), \operatorname{Sp}(\sigma, \Gamma))=N_{\operatorname{Sp}(2 n, R)}(\operatorname{Ep}(\sigma, \Gamma), \operatorname{Sp}(\sigma, \Gamma)),
$$

where the last equality is due to Theorem 2. Therefore we only need to prove that the sandwich inclusions in Theorem 1 are satisfied for the form net of ideals associated with the subgroup $H$ for a Noetherian ground ring.

Proof of Theorem 1; existence. The remark above justifies the assumption that $R$ is Noetherian. Let $(\sigma, \Gamma)$ be the form net of ideals associated with $H$, cf. Lemma 4.1. Pick an element $a$ in $H$, a $(\sigma, \Gamma)$-elementary transvection $T_{s r}(\xi)$, long or short, and 
denote by $b$ the corresponding root element $a T_{s r}(\xi) a^{-1}$. Our goal is to show that $b$ is contained in $\operatorname{Sp}(\sigma, \Gamma)$. For each $i, j \in I$ put

$$
\begin{aligned}
X_{i j} & =\left\{\xi \in R \mid \xi b_{i j} \in \sigma_{i j}\right\} \\
Z_{i} & =\left\{\xi \in R \mid \xi^{2} S_{i,-i}(b) \in \Gamma_{i}\right\} .
\end{aligned}
$$

We will show that the sets $X_{i j}$ and $Z_{i}$ are unimodular, i.e. generate the unit ideal $R$, for all $i, j \in I$. Fix a maximal ideal $\mathfrak{m}$ of $R$ and let $S$ denote the compliment $R \backslash \mathfrak{m}$ of $\mathfrak{m}$ in $R$. Let $R_{\mathfrak{m}}$ denote the localization $S^{-1} R$ of the ring $R$ at the multiplicative system $S$ and $F_{\mathfrak{m}}$ the corresponding localization morphism $R \rightarrow R_{\mathfrak{m}}$. Recall that for $\zeta \in R$ and $s \in S$ we denote $F_{\mathfrak{m}}(\zeta) \cdot F_{\mathfrak{m}}(s)^{-1}$ by $\frac{\zeta}{s}$. Let $R_{\mathfrak{m}}^{\prime}$ denote the image of $R$ under $F_{\mathfrak{m}}$ and $S_{\mathfrak{m}}$ denote the image of $S$ under $F_{\mathfrak{m}}$. We have already discussed at the end of Section 5 that $\left(R_{\mathfrak{m}}, R_{\mathfrak{m}}^{\prime}, S_{\mathfrak{m}}\right)$ is a standard setting. Let $H_{\mathfrak{m}}$ denote the image of $H$ under $\mathrm{M}\left(F_{\mathfrak{m}}\right)$. Clearly $\operatorname{Ep}\left(\nu, R_{\mathfrak{m}}^{\prime}\right) \leq H_{\mathfrak{m}}$.

We will show now that there exists an element $x_{0} \in S$ such that $F_{\mathfrak{m}}$ is injective on $x_{0} R$. In fact, this is exactly the point of the Noetherian reduction. For an arbitrary ground ring this fail to be true in general. For each $x \in S$ set $\operatorname{Ann}(x)=\{\xi \in R \mid x \xi=0\}$. As $R$ is Noetherian, the set of ideals $\{\operatorname{Ann}(x) \mid x \in S\}$ contains a maximal element $\operatorname{Ann}\left(x_{0}\right)$. Let $\xi x_{0}, \zeta x_{0}$ be two arbitrary elements of $x_{0} R$. Suppose $F_{\mathfrak{m}}\left(\xi x_{0}\right)=F_{\mathfrak{m}}\left(\zeta x_{0}\right)$. Then there exists an element $y \in S$ such that $y x_{0}(\xi-\zeta)=0$. Therefore $\xi-\zeta \in \operatorname{Ann}\left(y x_{0}\right) \geq \operatorname{Ann}\left(x_{0}\right)$ and by the maximality of $\operatorname{Ann}\left(x_{0}\right)$ it follows that $\xi-\zeta \in A n n\left(x_{0}\right)$. Consequently $\xi x_{0}=\zeta x_{0}$. Therefore the localization morphism $F_{\mathfrak{m}}$ is injective on $x_{0} R$. This allows us to lift transections from $H_{\mathfrak{m}}$ to $H$ up to multiplication of the parameter by an element of $S$.

Proposition 9.1. Let $\zeta \in R$ and $x \in S$. If $T_{p q}\left(\frac{\zeta}{x}\right) \in H_{\mathfrak{m}}$ then

$$
T_{p q}\left(x_{0}^{\left(1+\delta_{p,-q}\right)} \cdot x^{\delta_{p,-q}} \cdot \zeta\right) \in H
$$

Proof. If $p \sim q$, the inclusion $T_{p q}\left(x_{0}^{\left(1+\delta_{p,-q}\right)} \cdot x^{\delta_{p,-q}} \cdot \zeta\right) \in H$ is trivial. Assume $p \nsim q$ and $p \neq-q$. There exists another index $h \sim q$ such that $h \neq \pm p, \pm q$. By the Steinberg relation $(\mathrm{R} 4)$

$$
T_{p q}\left(F_{\mathfrak{m}}(\zeta)\right)=\left[\left[T_{p q}\left(\frac{\zeta}{x}\right), T_{q h}\left(\frac{x}{1}\right)\right], T_{h q}(1)\right] \in H_{\mathfrak{m}} .
$$

Pick any pre-image $g$ of the matrix $T_{p q}\left(F_{\mathfrak{m}}(\zeta)\right)$ contained in $H$. Note, that $g$ neither has to coincide with $T_{p q}(\zeta)$, nor has to be a transvection at all. Nevertheless, by (R4) we get

$$
T_{p q}\left(F_{\mathfrak{m}}\left(\zeta x_{0}\right)\right)=\mathrm{M}\left(F_{\mathfrak{m}}\right)\left(\left[\left[g, T_{q h}\left(x_{0}\right)\right], T_{h q}(1)\right]\right) \in \mathrm{M}\left(F_{\mathfrak{m}}\right)\left(\operatorname{Sp}\left(2 n, R, x_{0} R\right) \cap H\right) .
$$

As $\mathrm{M}\left(F_{\mathfrak{m}}\right)$ is injective on $\operatorname{Sp}\left(2 n, R, x_{0} R\right)$, it follows that $T_{p q}\left(\zeta x_{0}\right) \in H$.

Assume $q=-p$ and $p \nsim-p$. Pick two more indices $h, t \in I$ such that $(p, h, t)$ is an A-type base triple. By the Steinberg relations (R3), (R4) and (R6) we get

$$
\begin{aligned}
T_{h,-h}\left(-\varepsilon_{p} \varepsilon_{h} F_{\mathfrak{m}}(\zeta x)\right)= & {\left[T_{p,-p}\left(\frac{\zeta}{x}\right), T_{-p,-h}\left(\frac{x}{1}\right)\right] } \\
& \times\left[T_{p t}(-1),\left[T_{t p}(1),\left[T_{p,-p}\left(\frac{\zeta}{x}\right), T_{-p,-h}\left(\frac{x}{1}\right)\right]\right]\right] \in H_{\mathfrak{m}} .
\end{aligned}
$$


As before, pick any pre-image $g$ of $T_{h,-h}\left(-\varepsilon_{p} \varepsilon_{h} F_{\mathfrak{m}}(\zeta x)\right)$, which is contained in $H$. Then by the Steinberg relations (R3), (R4) and (R6)

$$
\begin{aligned}
T_{p,-p}\left(F_{\mathfrak{m}}\left(\zeta x x_{0}^{2}\right)\right) & =\mathrm{M}\left(F_{\mathfrak{m}}\right)\left(\left[g, T_{-h,-p}\left(x_{0}\right)\right]\left[T_{h t}(-1),\left[T_{t h}(1),\left[g, T_{-h,-p}(1)\right]\right]\right]\right) \\
& \in \mathrm{M}\left(F_{\mathfrak{m}}\right)\left(\operatorname{Sp}\left(2 n, R, x_{0} R\right) \cap H\right) .
\end{aligned}
$$

By the injectivity of $\mathrm{M}\left(F_{\mathfrak{m}}\right)$ on $\operatorname{Sp}\left(2 n, R, x_{0} R\right)$ it follows that $T_{p,-p}\left(\zeta x x_{0}^{2}\right) \in H$.

Let $\left(\sigma_{\mathfrak{m}}^{\prime}, \Gamma_{\mathfrak{m}}^{\prime}\right)$ denote the coordinate-wise image of $(\sigma, \Gamma)$ under $F_{\mathfrak{m}}$. It is easy to see that $\left(\sigma_{\mathfrak{m}}^{\prime}, \Gamma_{\mathfrak{m}}^{\prime}\right)$ is an exact major form net of ideals over $R_{\mathfrak{m}}^{\prime}$. Proposition 9.1 allows us to conclude that $\left(\sigma_{\mathfrak{m}}^{\prime}, \Gamma_{\mathfrak{m}}^{\prime}\right)$ is $S_{\mathfrak{m}}$-associated, cf. Section 5, with $H_{\mathfrak{m}}$. Indeed, the inclusion $\operatorname{Ep}\left(\sigma_{\mathfrak{m}}^{\prime}, \Gamma_{\mathfrak{m}}^{\prime}\right) \leq H_{\mathfrak{m}}$ is obvious. Suppose $T_{p q}\left(\frac{\xi}{x}\right) \in H_{\mathfrak{m}}$ for some elementary transvection $T_{p q}\left(\frac{\xi}{x}\right)$ in $\operatorname{Ep}\left(2 n, R_{\mathfrak{m}}\right)$. By Proposition 9.1 it follows that $T_{p q}\left(\xi x^{\delta_{p,-q}} x_{0}^{\left(1+\delta_{p,-q}\right)}\right) \in H$. Thus $\xi x^{\delta_{p,-q}} x_{0}^{\left(1+\delta_{p,-q}\right)} \in(\sigma, \Gamma)_{p q}$ and $F_{\mathfrak{m}}(\xi) F_{\mathfrak{m}}\left(x^{\delta_{p,-q}} x_{0}^{\left(1+\delta_{p,-q}\right)}\right) \in\left(\sigma_{\mathfrak{m}}^{\prime}, \Gamma_{\mathfrak{m}}^{\prime}\right)_{p q}$. Therefore $\left(\sigma_{\mathfrak{m}}^{\prime}, \Gamma_{\mathfrak{m}}^{\prime}\right)$ is indeed $S_{\mathfrak{m}}$-associated with $H_{\mathfrak{m}}$.

Let $\left(\sigma_{\mathfrak{m}}, \Gamma_{\mathfrak{m}}\right)$ denote the $S_{\mathfrak{m}}$-closure of $\left(\sigma_{\mathfrak{m}}^{\prime}, \Gamma_{\mathfrak{m}}^{\prime}\right)$ in $R_{\mathfrak{m}}$. It follows by Theorem 8.1 that $\mathrm{M}\left(F_{\mathfrak{m}}\right)(b) \in \operatorname{Sp}\left(\sigma_{\mathfrak{m}}, \Gamma_{\mathfrak{m}}\right)$.

Now we will show that each set $X_{i j}$ and $Z_{i}$ contains an element of $S$ and thus each is not contained in $\mathfrak{m}$. For $i \sim j$ it is easy to see that $X_{i j}=R$, therefore we may assume that $i \nsim j$. Let $i \neq-j$. As $F_{\mathfrak{m}}\left(b_{i j}\right) \in\left(\sigma_{\mathfrak{m}}\right)_{i j}$, there exists an element $x \in S$ such that $T_{i j}\left(F_{\mathfrak{m}}\left(b_{i j} x\right)\right) \in H_{\mathfrak{m}}$. By Proposition 9.1 it follows that $b_{i j} x x_{0} \in \sigma_{i j}$ which means that $x x_{0} \in X_{i j}$. If $j=-i$ then there exists an index $k \sim i$ such that $i \neq \pm k$. Therefore $F_{\mathfrak{m}}\left(b_{i,-i}\right) \in\left(\sigma_{\mathfrak{m}}\right)_{i,-i}=\left(\sigma_{\mathfrak{m}}\right)_{i,-k}$. Exactly as in the previous case we get that $b_{i j} x x_{0} \in \sigma_{i,-k}=\sigma_{i,-i}$ for some $x \in S$. Finally, assume $i \nsim-i$. As $F_{\mathfrak{m}}\left(S_{i,-i}(b)\right) \in\left(\Gamma_{\mathfrak{m}}\right)_{i}$, there exists an element $x \in S$ such that $T_{i,-i}\left(F_{\mathfrak{m}}\left(S_{i,-i}(b) x^{2}\right)\right) \in H_{\mathfrak{m}}$. By Proposition 9.1 it follows that $T_{i,-i}\left(F_{\mathfrak{m}}\left(S_{i,-i}(b) x^{2} x_{0}^{2}\right)\right) \in H$, which yields that $x_{0} x \in Z_{i}$.

Fix some indices $i$ and $j$. We have shown that the set $X_{i j}$ is unimodular, therefore $b_{i j} \in\left\langle X_{i j}\right\rangle b_{i j} \leq \sigma_{i j}$. Thus $b \in \operatorname{Sp}(\sigma)$. We have also shown that the set $Z_{i}$ is unimodular. Therefore there exist elements $\zeta_{1}, \ldots, \zeta_{k} \in Z_{i}$ and elements $\xi_{1}, \ldots, \xi_{k} \in R$ such that $\sum_{t=1}^{k} \xi_{t} \zeta_{t}=1$. Thus $\sum_{t=1}^{k} \xi_{t}^{2} \zeta_{t}^{2} \equiv 1 \bmod 2 R$ and

$$
S_{i,-i}(b) \in \sum_{t=1}^{k} \xi_{t}^{2}\left(\zeta_{t}^{2} S_{i,-i}(b)\right)+2 R S_{i,-i}(b) \leq \Gamma_{i}+2 \sigma_{i,-i} \leq \Gamma_{i} .
$$

Summing up, $b \in \operatorname{Sp}(\sigma, \Gamma)$. This completes the proof of existence of such an exact form net of ideals $(\sigma, \Gamma)$ that

$$
\operatorname{Ep}(\sigma, \Gamma) \leq H \leq \operatorname{Transp}_{\operatorname{Sp}(2 n, R)}(\operatorname{Ep}(\sigma, \Gamma), \operatorname{Sp}(\sigma, \Gamma)) .
$$

Proof of Theorem 1: uniqness. Now we will show that an exact form net of ideals $(\sigma, \Gamma)$ satisfying (49) is unique. Assume the contrary: let $(\tau, B)$ be an exact major form net over $(R, \Lambda)$ such that

$$
\operatorname{Ep}(\tau, B) \leq H \leq \operatorname{Transp}_{\mathrm{Sp}(2 n, R)}(\operatorname{Ep}(\tau, B), \operatorname{Sp}(\tau, B)),
$$


but $(\tau, B)$ is not equal to $(\sigma, \Gamma)$. As $(\sigma, \Gamma)$ is maximal among exact form nets such that $\operatorname{Ep}(\sigma, \Gamma) \leq H$, it follows that $(\tau, b) \leq(\sigma, \Gamma)$. Pick any $\xi \in(\sigma, \Gamma)_{i j}$. Then $T_{i j}(\xi) \in H \leq$ $\operatorname{Transp}_{\mathrm{Sp}(2 n, R)}(\operatorname{Ep}(\tau, B), \operatorname{Sp}(\tau, B))$. First, assume $i \neq-j$. By property (T1) of Theorem 2 applied to the net $(\tau, B)$ it follows, because $\left(T_{i j}(\xi)\right)_{j j}=1$, that

$$
\xi=\left(T_{i j}(\xi)\right)_{i j} \cdot 1 \cdot\left(T_{i j}(\xi)\right)_{j j} \leq\left(T_{i j}(\xi)\right)_{i j} \cdot \tau_{j j} \cdot\left(T_{i j}(\xi)\right)_{j j} \leq \tau_{i j}
$$

Therefore $\tau_{i j}=\sigma_{i j}$ for all $i \neq-j$. If $i=-j$ then by property (T2) of Theorem 2

$$
\xi=\left(T_{i,-i}(\xi)\right)_{i i}^{2} \cdot 1^{2} \cdot S_{i,-i}\left(T_{i,-i}(\xi)\right) \leq\left(T_{i,-i}(\xi)\right)_{i i}^{2} \cdot \tau_{i i}^{[2]} \cdot S_{i,-i}\left(T_{i,-i}(\xi)\right) \leq B_{i} .
$$

Therefore $B_{i}=\Gamma_{i}$ for all $i \in I$. Finally as both form nets $(\sigma, \Gamma)$ and $(\tau, B)$ are exact, it follows that $(\sigma, \Gamma)=(\tau, B)$.

\section{References}

[Asc84] M. Aschbacher, On the maximal subgroups of the finite classical groups, Invent. Math. 76 (1984), no. 3, 469-514.

[Asc85] M. Aschbacher, Subgroup structure of finite groups, Proceedings of the Rutgers group theory year, 1983-1984 (New Brunswick, N.J., 1983-1984), Cambridge Univ. Press, Cambridge, 1985, pp. 35-44.

[Asc86] M. Aschbacher, Finite simple groups and their subgroups, Group theory, Beijing 1984, Lecture Notes in Math., vol. 1185, Springer, Berlin, 1986, pp. 1-57.

[Bak69] A. Bak, The stable structure of quadratic modules, Ph.D. thesis, Columbia University, 1969.

[Bak81] A. Bak, K-theory of forms, Princeton University Press ; University of Tokyo Press, 1981.

[BS01] A. Bak and A. Stepanov, Dimension theory and non-stable K-theory for net subgroups, Rend. Sem. Mat. Univ. Padova 106 (2001), 207-253.

[BV82] Z. I. Borevich and N. A. Vavilov, Arrangement of subgroups that contain the group of block diagonal matrices in the general linear group over a ring, Izv. Vuzov, Mat 11 (1982), 11-15.

[BV84] Z. I. Borevich and N. A. Vavilov, The distribution of subgroups in the full linear group over a commutative ring, Proceedings of the Steklov Institute of Mathematics 165 (1984), 24-42.

[BV00] A. Bak and N. A. Vavilov, Structure of hyperbolic unitary groups I: Elementary subgroups, Algebra Colloquium 7 (2000), no. 2, 159-196. 
[BVN70] Z. I Borevich, N. A. Vavilov, and W. Narkiewicz, On subgroups of the general linear group over a Dedekind ring, Zap. Nauchn. Semin. LOMI 94 (1970), $13-20$.

[Dyb98] E. V. Dybkova, Form nets and the lattice of overdiagonal subgroups of the symplectic group over a field of characteristic 2, Algebra i Analiz 10 (1998), no. $4,113-129$.

[Dyb99] E. V. Dybkova, Overdiagonal subgroups of the hyperbolic unitary group for a good form ring over a field, Journal of Mathematical Sciences 95 (1999), no. 2, 2096-2101 (English).

[Dyb06] E. V. Dybkova, The Borevich theorem for the hyperbolic unitary group over a skew field, Journal of Mathematical Sciences 136 (2006), no. 3, 3908-3925.

[Dyb07] E. V. Dybkova, Overgroups of the diagonal group in the hyperbolic unitary group over a simple Artin ring. I, Journal of Mathematical Sciences 145 (2007), no. 1, 4781-4789.

[Dyb09] E. V. Dybkova, Overgroups of the diagonal group in the hyperbolic unitary group over a simple Artin ring. II, Journal of Mathematical Sciences 156 (2009), no. 6, 901-917 (English).

[Dyn57a] E. Dynkin, Maximal subgroups of classical groups, Amer. Math. Soc. Translations 6 (1957), no. 2, 245-378.

[Dyn57b] E. Dynkin, Semisimple subalgebras of semisimple Lie algebras, Amer. Math. Soc. Translations 6 (1957), 111-244.

[KL90] P. B. Kleidman and M. W. Liebeck, The subgroup structure of the finite classical groups, Cambridge University Press, 1990.

[Sei87] G. M. Seitz, The maximal subgroups of classical algebraic groups, Mem. Amer. Math. Soc. 67 (1987), 365.

[Shc15] A. Shchegolev, Overgroups of elementary block-diagonal subgroups in even unitary groups over quasi-finite rings, Ph.D. thesis, Universität Bielefeld, 2015.

[Shc17] A. V. Shchegolev, Overgroups of elementary block diagonal subgroups in even unitary groups over quasi-finite rings: Main results, Journal of Mathematical Sciences 222 (2017), no. 4, 516-523.

[Tes88] D. M. Testerman, Irreducible subgroups of exceptional algebraic groups, Mem 390 (1988).

[Vav83] N. A. Vavilov, The subgroups of the general linear group over a semilocal ring that contain the group of block diagonal matrices, Vestn. Leningr. Univ. 1 (1983), 16-21. 
[Vav87] N. A. Vavilov, On the subgroups of the general linear group over a dedekind ring of arithmetic type, Izv. Vuzov, Mat. 17 (1987), 14-20.

[Vav88] N. A. Vavilov, Subgroups of split orthogonal groups over a ring, Sib. Math. Zh. 29 (1988), 31-43.

[Vav93] N. A. Vavilov, Subgroups of the general symplectic group over a commutative ring, Rings and Modules. Limit Theorems in Probability Theory 1 (1993), $16-38$.

[Vav04] N. A. Vavilov, Subgroups of split orthogonal groups over a commutative ring, Journal of Mathematical Sciences 120 (2004), 1501-1512.

[Vav08] N. A. Vavilov, Subgroups of symplectic groups that contain a subsystem subgroup, Journal of Mathematical Sciences 151 (2008), 2937-2948.

[VS13] N. A. Vavilov and A. V. Shchegolev, Overgroups of subsystem subgroups in exceptional groups: Levels, Journal of Mathematical Sciences 192 (2013), no. 2, 164-195.

[You12] H. You, Subgroups of classical groups normalized by relative elementary groups, Journal of Pure and Applied Algebra 216 (2012), no. 5, 1040 - 1051. 